UNIVERSIDADE DE BRASÍLIA

FACULDADE DE TECNOLOGIA

DEPARTAMENTO DE ENGENHARIA CIVIL E AMBIENTAL

\section{META MODELOS PARA QUANTIFICAÇÃO DA INCERTEZA NA PREDIÇÃO DE FENÔMENOS DA MECÂNICA DA FRATURA E DA FADIGA}

IAGO FREITAS DE ALMEIDA

ORIENTADOR: FRANCISCO EVANGELISTA JUNIOR, PhD.

DISSERTAÇÃO DE MESTRADO EM ESTRUTURAS E CONSTRUÇÃO CIVIL

PUBLICAÇÃO: E.DM - 07A/17

BRASÍLIA/DF: MARÇO - 2017 
UNIVERSIDADE DE BRASÍLIA

FACULDADE DE TECNOLOGIA

DEPARTAMENTO DE ENGENHARIA CIVIL E AMBIENTAL

META MODELOS PARA QUANTIFICAÇÃO DA INCERTEZA NA PREDIÇÃO DE FENÔMENOS DA MECÂNICA DA FRATURA E DA FADIGA

IAGO FREITAS DE ALMEIDA

DISSERTAÇÃO SUBMETIDA AO DEPARTAMENTO DE ENGENHARIA CIVIL E AMBIENTAL DA FACULDADE DE TECNOLOGIA DA UNIVERSIDADE DE BRASÍLIA COMO PARTE DOS REQUISÍTOS NECESSÁRIOS PARA A OBTENÇÃO DO GRAU DE MESTRE EM ESTRUTURAS E CONSTRUÇÃO CIVIL.

APROVADA POR:

Prof. Francisco Evangelista Junior, PhD. (ENC-UnB) (Orientador)

Prof. Luciano Mendes Bezerra, PhD. (ENC-UnB)

(Examinador Interno)

Prof. Adriano Todorovic Fabro, PhD. (ENM-UnB)

(Examinador Externo)

BRASÍLIA/DF, 31 DE MARÇO DE 2017 


\section{FICHA CATALOGRÁFICA}

\begin{tabular}{|c|c|}
\hline $\begin{array}{r}\text { ALMEIDA, IAC } \\
\text { Meta Modelos } \mathrm{p} \\
\mathrm{f}\end{array}$ & ão de fenômenos da mecânica da \\
\hline $\begin{array}{l}\mathrm{xx}, 73 \mathrm{p} ., 210 \mathrm{x} \\
\text { Dissertação de } \mathrm{N} \\
\text { Departamento } \mathrm{de}\end{array}$ & $\begin{array}{l}\text { ras e Construção Civil, 2017). } \\
\text { uldade de Tecnologia. }\end{array}$ \\
\hline 1.Meta modelo & 2.Mecânica da fratura \\
\hline 3.Fadiga & 4.Quantifacação da incerteza \\
\hline I. ENC/FT/UnB & II. Título (Mestre) \\
\hline
\end{tabular}

\section{REFERÊNCIA BIBLIOGRÁFICA}

ALMEIDA, I. F. (2017). Meta Modelos para Quantificação da Incerteza na Predição de Fenômenos da Mecânica da Fratura e da Fadiga. Dissertação de Mestrado em Estruturas e Construção Civil, Publicação E.DM-07A/17, Departamento de Engenharia Civil e Ambiental, Universidade de Brasília, Brasília, DF, 73p.

\section{CESSÃO DE DIREITOS}

AUTOR: Iago Freitas de Almeida

TÍTULO: Meta Modelos para Quantificação da Incerteza na Predição de Fenômenos da Mecânica da Fratura e da Fadiga.

GRAU: Mestre ANO: 2017

É concedida à Universidade de Brasília permissão para reproduzir cópias desta dissertação de mestrado e para emprestar ou vender tais cópias somente para propósitos acadêmicos e científicos. $\mathrm{O}$ autor reserva outros direitos de publicação e nenhuma parte dessa dissertação de mestrado pode ser reproduzida sem autorização por escrito do autor.

Iago Freitas de Almeida

SQN 404, BL C, Térreo 02, Asa Norte.

70845-030 Brasília - DF - Brasil.

iago.freitas@hotmail.com 


\section{AGRADECIMENTOS}

O fim de uma etapa é sempre difícil, porém é necessário para que só assim possamos concluir nossos objetivos. O mestrado é para muitos uma etapa difícil, principalmente para aqueles que acabaram de se graduar. Além disso, é importante relatar que nunca devemos desistir de nossos sonhos, pois mesmo nas dificuldades, a luz do conhecimento mantém-se acessa. Desta forma, chego ao final deste tão sonhado curso de pós-graduação e vejo-me que ao longo desta etapa, muitos amigos, colegas e principalmente a família foram de fundamental importância para meu sucesso. Contudo, mesmo não sendo suficientes estes agradecimentos, tentarei ser breve, pois nunca me esquecerei deste momento.

Agradeço este trabalho em primeiro lugar ao meu tão amado Deus que de uma forma excepcional ajudou-me a chegar tão longe. A mágica de Deus está presente em tudo, mesmo que não sejamos capazes de ver, ela encanta e sempre estará a encantar novas gerações. Em segundo plano a minha família que sempre esteve ao meu lado em todos os momentos.

Aos meus pais, Ely e Luiz Junior, pelo constante apoio, principalmente, nos momentos em que a saudade era grande. Aos meus irmãos Igor e Raul, por incentivarem sempre a minha caminhada. Aos meus avôs, Esmeraldina e Luiz que mesmo distantes foram importantes neste momento.

Ao meu orientador, pela orientação objetiva, segura e competente. A todos os professores do Programa de Pós-Graduação em Estruturas e Construção Civil que de alguma forma contribuíram para este trabalho.

Aos amigos neste período de mestrado: Álvaro Martins, Adeilson Sousa, Carlos Valbson, Carlos Eduardo, Dyaloisio Fonteles, Denise Cardoso, Eduardo Pains, Eduardo Martins, Fabiano Campos, Fernando Hipólito, Fellipe Sobreira, Gelson Alves, Guilherme Vieralves, Guilherme Oliveira, Samara Pimentel, Izabel Castro, Jéssica Borges, Jéssica Siqueira, Manuel Alejandro, Nelson Afanador, Nathaly Sarasty, José Fabiano, Nicolás Rojas e Thiarly Lavôr. Aos amigos da Federação Espírita Brasileira (Brasília) pelo apoio e orações prestadas durante este momento.

Ao CNPq, pelo apoio financeiro. 
"Tudoé imp ortante na vida. Os pequenos atos são preparatóiios dos gestos grandiosos e das realizações vultosas".

(Joanna de Ângelis)

Dedico à memória de minha avó, Maria de Jesus, pelo apoio para realização dos meus sonhos e por ter sido uma excelente mãe. 


\section{RESUMO}

\section{META MODELOS PARA QUANTIFICAÇÃO DA INCERTEZA NA PREDIÇÃO DE FENÔMENOS DA MECÂNICA DA FRATURA E DA FADIGA}

Autor: Iago Freitas de Almeida

Orientador: Francisco Evangelista Junior

Programa de Pós-graduação em Estruturas e Construção Civil

Brasília, março de 2017

Esta dissertação tem como objetivo formular e implementar meta modelos polinomiais e funções de base radial para quantificação de incerteza de fenômenos selecionados da mecânica da fratura e fadiga. Na mecânica da fratura, meta modelos com amostragens de Sequência de Sobol e Hipercubo Latino foram utilizados para análise da taxa de liberação de energia de uma placa de concreto com uma trinca semielíptica e com envelhecimento viscoelástico do material. Já para a análise da fadiga, meta modelos com amostragens Hipercubo Latino, Sequência de Sobol e Experimento Fatorial foram considerados para o número de ciclos de vida à fadiga do material. Foi proposto também um algoritmo que incorpora simulação de Monte Carlo no meta modelo capaz de considerar variáveis normais e não normais para quantificação da incerteza. Os resultados obtidos comprovam a eficiência e acurácia dos modelos tanto em superfícies polinomiais como nos modelos com funções de base radial. Ademais, os meta modelos conseguem fornecer resultados com boa precisão ao dos modelos de referência dados pela literatura com um número de amostras reduzidas para construção do meta modelo.

Palavras chave: Meta modelo, Mecânica da fratura, Fadiga, Quantificação da incerteza. 


\section{ABSTRACT \\ METAMODELS FOR UNCERTAINTY QUANTIFICATION IN PREDICTION OF FRACTURE MECHANICS AND FATIGUE PHENOMENA}

Author: Iago Freitas de Almeida

Supervisor: Francisco Evangelista Junior

Programa de Pós-graduação em Estruturas e Construção Civil

Brasilia, March 2017

This research aims to formulate and implement polynomial and radial basis function metamodels for uncertainty quantification in selected fracture mechanics and fatigue problems. In fracture mechanics, metamodels with samples of Latin Hypercube and Sobol Sequence were used to analyze the energy release rate of a concrete slab with a semielliptical crack and with material viscoelastic aging. For the analysis of fatigue, metamodels with samples of Latin Hypercube, Sobol Sequence and Factorial Design were considered for the number of life cycles in fatigue of the material. It was also proposed an algorithm that incorporates Monte Carlo simulation in metamodels capable of considering normal and non-normal variables for the uncertainty quantification. The results obtained proved the efficiency and accuracy of the models in polynomial surfaces and in radial basis functions models. In addition, the metamodels could provide results with good precision to the reference models of the literature with a reduced number of samples to construct the metamodel.

Keywords: Metamodel, Fracture Mechanics, Fatigue, Uncertainty Quantification. 


\section{SUMÁRIO}

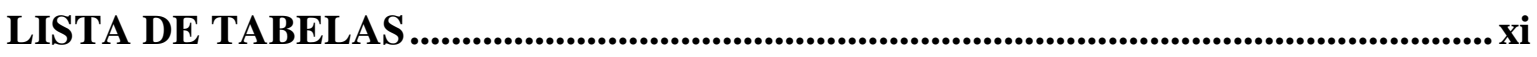

LISTA DE FIGURAS........................................................................................

LISTA DE SÍMBOLOS, NOMENCLATURA E ABREVIAÇÕES ............................ xiv

1. INTRODUÇÃ

1.1. MOTIVAÇÃ

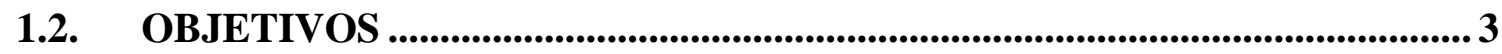

1.2.1. Objetivos gerais ............................................................................................... 3

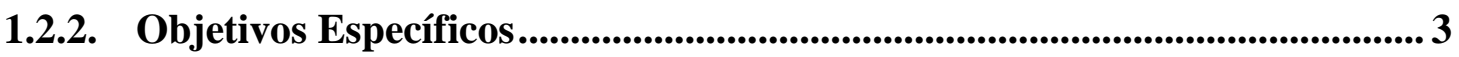

1.3. CONTRIBUIÇÕES DO TRABALHO ..................................................4

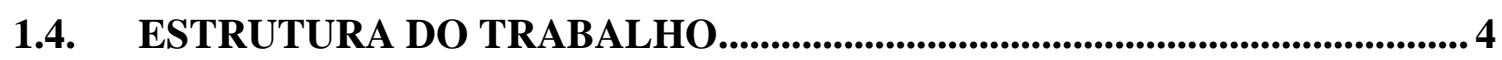

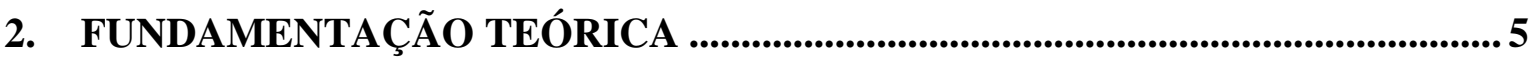

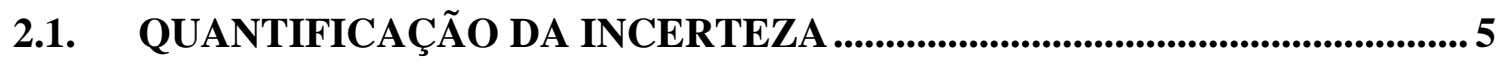

2.1.1. Distribuição de probabilidade ...............................................................................5

2.1.2. Simulação de Monte Carlo.............................................................................8

2.1.3. Geração de números aleatórios ........................................................... 8

2.1.4. Transformação para espaço normal padrão................................................9

2.2. META MODELOS OU META MODELAGEM ............................................ 10

2.2.1. Técnicas de Amostragem ...................................................................................... 10

2.2.2. Superfície de Resposta .................................................................. 12

3. QUANTIFICAÇÃO DA INCERTEZA DA VIDA DE FADIGA DE BAIXO CICLO EM MEMBROS ESTRUTURAIS ............................................................. 19

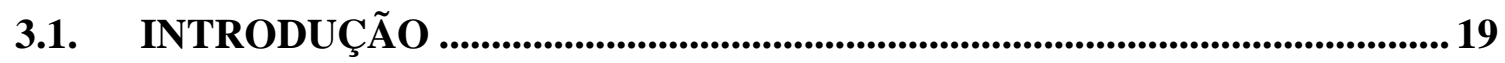

3.2. ESTUDO DE CASO EM PLACA METÁLICA ............................................... 20

3.2.1. Parâmetros do Modelo ........................................................................................... 20

3.2.2. Construção dos meta modelos ............................................................................ 22

3.3. ANÁLISE DE RESULTADOS................................................................ 25

4. QUANTIFICAÇÃO DA INCERTEZA DA TAXA DE LIBERAÇÃO DE ENERGIA DEPENDENTE DO TEMPO DE UM MEMBRO ESTRUTURAL COM

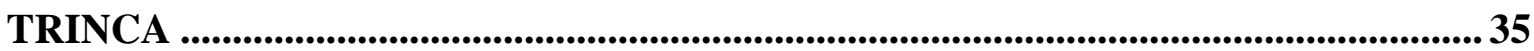

4.1. INTRODUÇÃ 
4.2. ESTUDO DE CASO DE PLACA 3D ........................................................36

4.2.1. Parâmetros do modelo ..............................................................................................36

4.2.2. Construção dos meta modelos ................................................................... 42

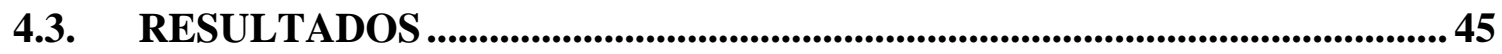

4.3.1. $\mathrm{G}_{\mathrm{I}}$ para $\mathrm{tc}=1$ dia $\mathrm{e} t=11$ dias ...................................................................... 46

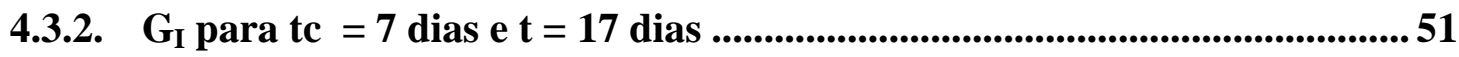

4.3.3. G $_{\text {I }}$ para $t c=14$ dias $\mathrm{t} t=24$ dias .................................................................55

4.3.4. Análise da variação de $\delta$.........................................................................................58

4.3.5. Análise Geral de $G_{I}$...............................................................................................59

5. CONCLUSÕES E SUGESTÕES PARA TRABALHOS FUTUROS ....................63

5.1. CONCLUSÕES........................................................................................63

5.2. SUGESTÕES PARA TRABALHOS FUTUROS .............................................64

REFERÊNCIAS BIBLIOGRÁFICAS ..........................................................................65

APÊNDICE A - PARÂMETROS DE REGRESSÃO PARA SEGUNDO CASO DE

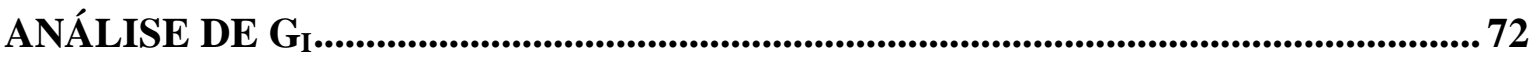

APÊNDICE B - PARÂMETROS DE REGRESSÃO PARA TERCEIRO CASO DE

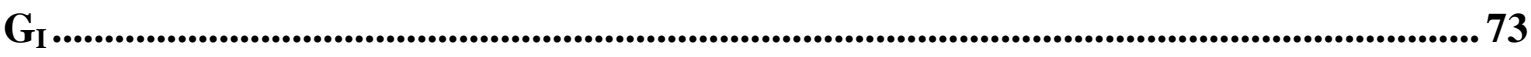




\section{LISTA DE TABELAS}

Tabela 3.1 - Valor das variáveis determinísticas à fadiga ................................................. 24

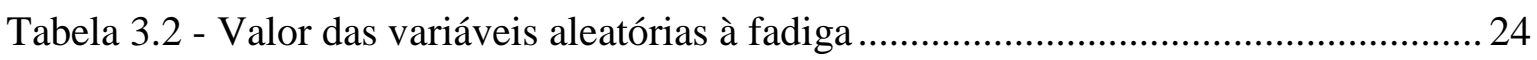

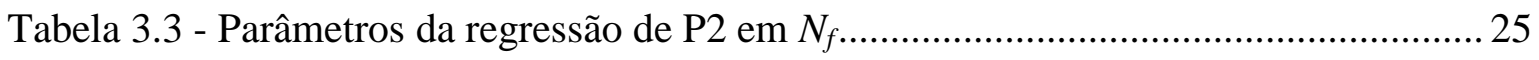

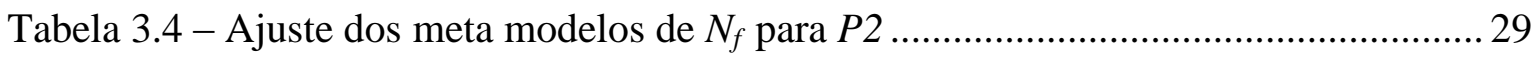

Tabela 3.5 - Parâmetros com 2, 3, 4 desvios padrões da regressão de P2 ........................... 30

Tabela 3.6 - Parâmetros com 2 e 3 desvios padrões com $R B F$......................................... 31

Tabela 3.7 - Parâmetros com 2 e 3 desvios padrões com RBFP1 ….................................. 31

Tabela 3.8 - Ajuste dos meta modelos de $N_{f}$ para $R B F$.................................................. 31

Tabela 3.9 - Ajuste dos meta modelos de $N_{f}$ para $R B F P 1$............................................... 32

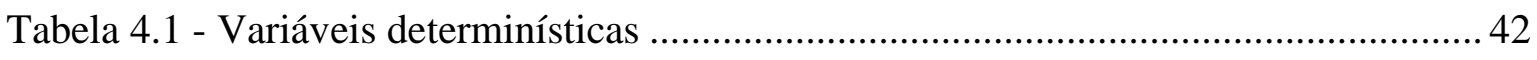

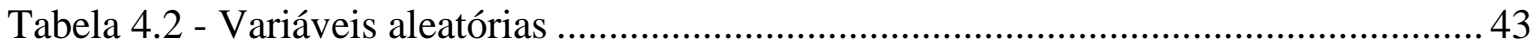

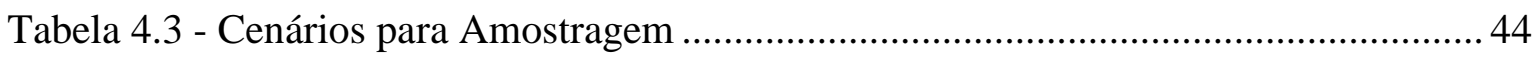

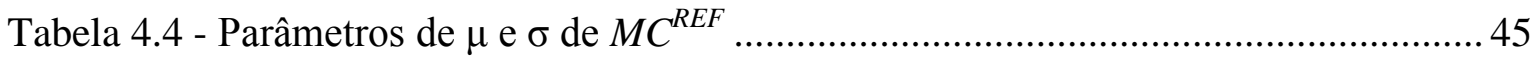

Tabela 4.5 - Parâmetros de P2 para $t_{c}=1$ dia e $t=11$ dias ................................................... 46

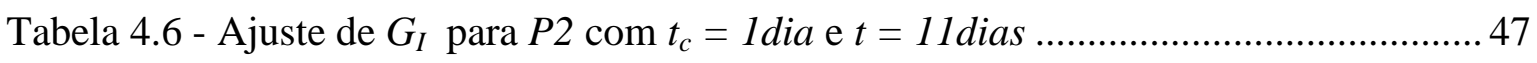

Tabela 4.7 - Parâmetros de $R B F$ para $t_{c}=1$ dia e $t=11$ dias ............................................... 48

Tabela 4.8 - Ajuste de $G_{I}$ para $R B F$ com $t_{c}=1$ dia e $t=11$ dias...................................... 48

Tabela 4.9 - Parâmetros de $R B F P 1$ para $t_{c}=1$ dia e $t=11$ dias ........................................... 49

Tabela 4.10 - Ajuste de $G_{I}$ para $R B F P 1$ com $t_{c}=1$ dia e $t=11$ dias ..................................50

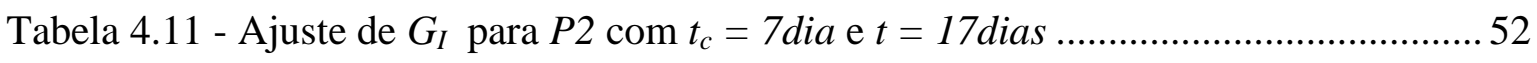

Tabela 4.12 - Ajuste de $G_{I}$ para $R B F$ com $t_{c}=7$ dia e $t=17$ dias ......................................53

Tabela 4.13 - Ajuste de $G_{I}$ para RBFP1 com $t_{c}=7$ dia e $t=17$ dias ..................................54

Tabela 4.14 - Ajuste de $G_{I}$ para $P 2 \operatorname{com} t_{c}=14$ dias e $t=24$ dias .....................................56

Tabela 4.15 - Ajuste de $G_{I}$ para $R B F$ com $t_{c}=14$ dias e $t=24$ dias ...................................5 57

Tabela 4.16 - Ajuste de $G_{I}$ para RBFPl com $t_{c}=14$ dias e $t=24$ dias ..............................58

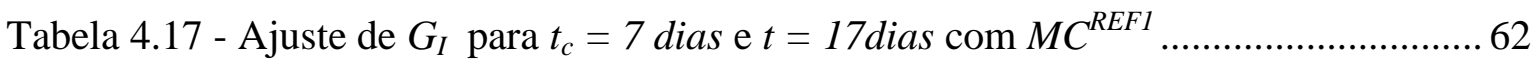

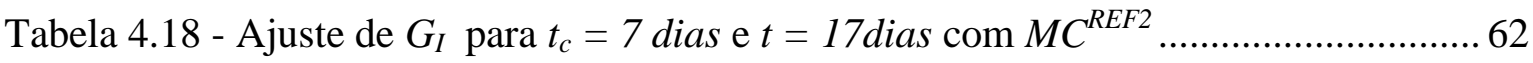




\section{LISTA DE FIGURAS}

Figura 2.1 - Função densidade de probabilidade (PDF) normal......................................... 6

Figura 2.2 - Função densidade de probabilidade $(P D F)$ de $G E V$...................................... 7

Figura 2.3 - Método Inverso da CDF (Adaptado de: Choi et al., 2007).............................. 9

Figura 2.4 - Projeto de Experimento Fatorial ...................................................................... 11

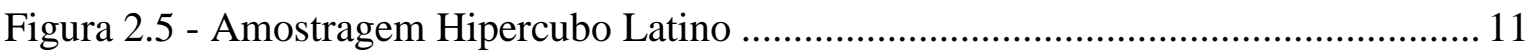

Figura 2.6 - Amostragem Sequência de Sobol .............................................................. 12

Figura 2.7 - Exemplo de superfície de resposta polinomial .............................................. 14

Figura 2.8 - Exemplo Superfície de Resposta para $\mathrm{Wu}-\mathrm{C} 2$............................................ 17

Figura 2.9 - Exemplo de resposta para Wendland - C2 ................................................ 18

Figura 3.1 - Placa com furo sob força axial (a) e história de tensão aplicada (b) ................ 20

Figura 3.2 - Fluxograma para construção do meta modelo ………...................................... 22

Figura 3.3 - Fluxograma para quantificação da incerteza em fadiga................................. 23

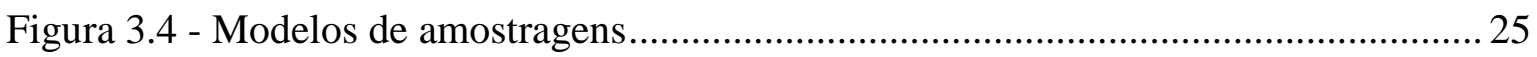

Figura 3.5 - Superfície de Resposta de P2 para (a) e (b) com FF ${ }^{\mathrm{II}}$ e para (c) e (d) com $\mathrm{CCD}^{\mathrm{II}}$ 26

Figura 3.6 - Superfície de Resposta de $P 2$ para (a) e (b) com FF ${ }^{I / I I}$ e para (c) e (d) com $\mathrm{CCD}^{\mathrm{I} / \mathrm{II}}$

Figura 3.7 - Superfície de Resposta de $P 2$ para (a) e (b) $\operatorname{LHS}^{\mathrm{II}}$ o e para (c) e (d) com SS 28 Figura 3.8 - $P D F$ s de $N_{f}$ para $P 2$ com (a) $\mathrm{FF}^{\mathrm{II}}$ e $\mathrm{CCD}^{\mathrm{II}}$, (b) $\mathrm{FF}^{\mathrm{I} / \mathrm{II}}$ e $\mathrm{CCD}^{\mathrm{I} / \mathrm{II}}$, (c) $\mathrm{LHS}^{\mathrm{II}}$ e $\mathrm{SS}^{\mathrm{II}}$, (d) LHS $^{\text {III }}$ e SS ${ }^{\text {III }}$. .29

Figura 3.9 - PDFs para (a) e (b) como $R B F$ e para (c) e (d) como RBFP1 ......................... 32

Figura 3.10 - Superfície de Resposta para $R B F$ com (a) e (b) $L H S^{\text {II }}$ e com (c) e (d) $S S^{\text {II }}$... 33 Figura 3.11 - Superfície de Resposta para RBFP1 para (a) e (b) LHS e para (c) e (d) SS .. 33 Figura 4.1 - Geometria para (a) placa com trinca no eixo original, (b) Modo I, (c) Modo II e (d) Detalhe da trinca 36

Figura 4.2 - Função fluência regredida, $J\left(t, t_{c}\right)$, e dados experimentais para diferentes $t_{c} . .40$ Figura 4.3 - Componente Instantânea, $J_{0}\left(t_{c}\right)$......

Figura 4.4 - Função fluência, $J\left(t, t_{c}\right)$, para os dias $t_{c}$ de carregamento ............................... 41

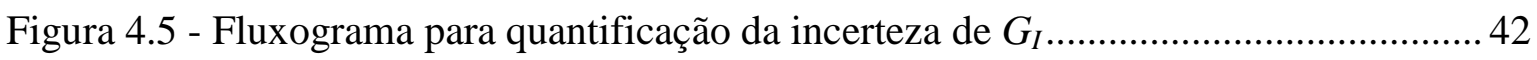

Figura 4.6 Média da taxa de liberação de energia, $\mu_{\mathrm{GI}(\mathrm{t}, \mathrm{tc})}$, utilizando $\mu$ das variáveis aleatórias 43

Figura 4.7 - Cenários de amostragem 44 
Figura 4.8 - PDFs de $M C^{R E F}$ para $G_{I}$

Figura 4.9 - PDFs de $G_{I}$ sendo (a) e (b) com N =79 e (c) e (d) com N = 200 para $P 2 \operatorname{com} t_{c}$

$=1$ dia e $t=11$ dias.

Figura 4.10 - PDFs de $G_{I}$ sendo (a) e (b) com N = 79 e (c) e (d) com N = 200 para RBF com $\mathrm{t}_{\mathrm{c}}=1$ dia e $\mathrm{t}=11$ dias.

Figura 4.11 - PDFs de $G_{I}$ sendo (a) e (b) com N=79 e (c) e (d) com N = 200 para RBFP1 com $\mathrm{t}_{\mathrm{c}}=1$ dia e $\mathrm{t}=11$ dias.

Figura $4.12-P D F s$ de $G_{I}$ para $C_{I I I} \operatorname{com} N=79$ e para $\mathrm{t}_{\mathrm{c}}=1$ dia e $\mathrm{t}=11$ dias

Figura 4.13 - $P D F s$ de $G_{I}$ sendo (a) e (b) $\operatorname{com} N=79$ e (c) e (d) $\operatorname{com} N=200$ para $P 2$ com $\mathrm{t}_{\mathrm{c}}=7$ dia e $\mathrm{t}=17$ dias.

Figura 4.14 - $P D F s$ de $G_{I}$ sendo (a) e (b) $\operatorname{com} N=79$ e (c) e (d) $\operatorname{com} N=200$ para $R B F$ com $t_{c}=7$ dia e $t=17$ dias.

Figura 4.15 - PDFs de $G_{I}$ sendo (a) e (b) $\operatorname{com} N=79$ e (c) e (d) com $N=200$ para $R B F P 1$ com $t_{c}=7$ dia e $t=17$ dias.

Figura 4.16 - $P D F s$ de $G_{I}$ sendo (a) e (b) $\operatorname{com} N=79$ e (c) e (d) $\operatorname{com} N=200$ para $P 2$ com $t_{c}=14$ dias e $t=24$ dias. .55

Figura 4.17 - PDFs de $G_{I} \operatorname{sendo}($ a) e (b) $\operatorname{com} N=79$ e (c) e (d) $\operatorname{com} N=200$ para $R B F$ com $t_{c}=14$ dias e $t=24$ dias.

Figura 4.18 - PDFs de $G_{I} \operatorname{sendo}($ a) e (b) $\operatorname{com} N=79$ e (c) e (d) com $N=200$ para $R B F P 1$ $\operatorname{com} t_{c}=14$ dias e $t=24$ dias.

Figura $4.19-P D F s$ de $G_{I} \operatorname{com} N=79$ para análise de $\delta$ para $R B F \operatorname{com} t_{c}=1$ dia e $t=11$

dias; sendo (a) $\delta=0,5$; (b) $\delta=1,0$ e (c) $\delta=2,0$. .58

Figura $4.20-P D F s$ de $G_{I} \operatorname{com} N=79$ para análise de $\delta$ para $R B F P 1 \operatorname{com} t_{c}=1$ dia e $t=$ 11 dias; sendo (a) $\delta=0,5$; (b) $\delta=1,0$ e (c) $\delta=2,0$. . . .

Figura $4.21-P D F s$ de $G_{I}$ para $C_{I I} \operatorname{com} N=79$ sendo (a) $t_{c}=1$ dia e $t=11$ dias; (b) $t_{c}=7$ dias e $t=17$ dias e (c) $t_{c}=14$ dias e $t=24$ dias.

Figura 4.22 - PDFs de $G_{I}$ para $C_{I I} \operatorname{com} N=200$ sendo (a) $t_{c}=1$ dia e $t=11$ dias; (b) $t_{c}=7$ dias e $t=17$ dias e (c) $t_{c}=14$ dias e $t=24$ dias. 61

Figura $4.23-P D F s$ de $G_{I}$ sendo com $t_{c}=7$ dias e $t=17$ dias. 61 


\section{LISTA DE SÍMBOLOS, NOMENCLATURA E ABREVIAÇÕES}

$a$

$b_{o}, b_{1}, \ldots, b_{n}$

$c_{o}, c_{1}, \ldots, c_{n}$

$b_{k}, c_{k}$

$c$

$\mathbf{A}_{\mathbf{o}}$

$A, B, \ldots, H \quad$ Histórico de tensão em uma placa metálica com entalhe

$d, g, p \quad$ Parâmetro de análise de trinca elíptica

$e$

$e_{r}$

$\mathrm{E}_{\mathrm{m}}$

$f_{t}, f_{b,} f_{\phi a}, f_{w}$

$f(x)$

$f_{s}$

F

$F(x)$

$G_{I}$

$G_{1}, G_{2}, Q$

$\mathrm{I}_{\mathrm{S}}$

$\mathrm{H}^{\prime}$

$H_{1}, H_{2}, H_{p}$

$J\left(t, t_{c}\right)$

$J_{o}$

$k$

Profundidade da trinca elíptica

Coeficientes de regressão polinomial de Lagrange

Coeficientes de interpolação de funções de base radial

Coeficientes de regressão log-log à fadiga

Metade do comprimento da trinca elíptica

Matriz modelo de regressão polinomial

Espessura da placa

Erro Residual de regressão polinomial

Módulo de elasticidade do material

Funções de análise de trinca elíptica 3D

Função Densidade de Probabilidade

Função real de integração do domínio $\mathrm{I}_{\mathrm{S}}$

Função de correção de trinca 3D

Função Cumulativa de Probabilidade

Taxa de liberação de energia em Modo I

Funções adicionais de análise de trinca 3D

Domínio de $[0,1]$ de cada dimensão de Sobol

Coeficiente de encruamento cíclico à fadiga

Funções complementares de análise de trinca 3D

Função Fluência

Componente Instantânea da função fluência

Fator do experimento fatorial 


\begin{tabular}{|c|c|}
\hline$K_{I}$ & Fator de Intensidade de Tensão no Modo I \\
\hline$K_{f}$ & Fator de concentração de tensão à fadiga \\
\hline$K_{\sigma}$ & Fator de concentração de tensão \\
\hline$K_{\varepsilon}$ & Fator de concentração de deformação \\
\hline$m$ & Número total de variáveis de entrada \\
\hline$M_{1}, M_{2}, M_{3}$ & Parâmetros da trinca elíptica \\
\hline$M_{s}$ & Momento aplicado a placa de concreto \\
\hline$M_{b}$ & Momento nos eixos principais de uma placa de concreto \\
\hline$n$ & Número total da amostragem por variável \\
\hline$n$ & Expoente de encruamento à fadiga \\
\hline$N(\mu, \sigma)$ & Distribuição Normal com média $\mu$ e desvio padrão $\sigma$ \\
\hline $\mathrm{N}$ & Número de amostras por superfície \\
\hline$N_{f}$ & Número de ciclos de vida à fadiga \\
\hline $\mathrm{p}_{\mathrm{o}}, \mathrm{W}_{1}, \mathrm{~W}_{2}$ & Parâmetros regredidos da função fluência \\
\hline $\mathrm{r}$ & Norma da variável de entrada \\
\hline $\mathrm{r}_{\mathrm{o}}$ & Ponto Central de uma função de base radial \\
\hline $\mathrm{r}^{2}$ & Coeficiente de determinação \\
\hline $\mathrm{R}$ & Raio do furo central em placa metálica \\
\hline $\mathrm{S}_{\mathrm{t}}$ & Soma dos resíduos totais entre os pontos de entrada e a média dos mesmos \\
\hline$t$ & Tempo de análise do concreto \\
\hline$t_{c}$ & Tempo de carregamento do concreto \\
\hline$u_{i}$ & Variável aleatória na distribuição uniforme entre 0 e 1 \\
\hline$x, x_{i}$ & Variável aleatória e/ou variável de entrada \\
\hline$x_{i}^{*}$ & Ponto de projeto \\
\hline$X_{1}, X_{2}, \ldots, X_{m}$ & Variável aleatória no espaço natural ou real \\
\hline$y$ & Função de saída ou variável dependente \\
\hline
\end{tabular}


$\mathrm{z}(\mathrm{N}(0,1)) \quad$ Coordenada equivalente no espaço normal padrão com média zero e desvio padrão igual a 1

$w$

Função de base radial

W

Comprimento da placa

\section{Símbolos Gregos}

$\beta$

$\Delta e$

Ângulo que indica a inclinação da trinca

$\Delta \varepsilon$

Variação de deformação nominal

$\Delta \sigma$

Variação de deformação na raiz do entalhe

$\Delta S$

Variação de tensão

$\delta$

Variação de tensão nominal

Parâmetro de suporte de uma função de base radial

$\delta_{o}$

Parâmetro de escala de uma distribuição de valor extremo generalizado

$\xi_{1}, \xi_{2}, \ldots, \xi_{m} \quad$ Variável aleatória no espaço codificado

$\rho$

Parâmetro de forma de uma função de base radial

$\varepsilon_{f}$

Coeficiente de ductilidade a fadiga

$\varepsilon_{a}$

Amplitude de deformação total

$\theta$

Diferença entre $t-t_{c}$

$\mu$

Valor esperado ou média

$\mu_{e q}$

Média Equivalente

$\mu_{y}$

Média ou valor esperado da função de saída

$\lambda$

Parâmetro de localização de uma distribuição $G E V$

$\sigma$

Desvio padrão populacional

$\sigma_{b}$ 
Desvio padrão equivalente

Tensão induzida por $M_{s}$ no plano inicial da trinca

Variância da função de saída

Tensão nominal no plano inicial da trinca

Tensão média

Tensão nominal no plano principal da trinca

Coeficiente de resistência à fadiga

Tensão cisalhante do Modo II no plano principal

Função densidade de probabilidade normal padrão

Inclinação angular que descreve a posição na frente da superfície da trinca

Parâmetro regredido de função de fluência $J\left(t, t_{c}\right)$

Função cumulativa de probabilidade normal padrão

Parâmetro de forma de uma distribuição de valor extremo generalizado

Variável aleatória uniforme $[0,1]$

\section{Abreviaturas}

$C C D$

Center Composite Design

$C D F$

Cumulative Density Function

CSRBF

Compactly supported radial basis function

FF

Full Factorial

GEV

Generalized Extreme Value

LHS Latin Hypercube Sampling

$M C$

Monte Carlo

$M C^{R E F}$

Monte Carlo de Referência

MSR

Metodologia de superfície de resposta

NRMSE

Normalized Root Mean Square Error 
RMSE Root Mean Square Error

PDF Probability Density Function

RBF Radial Basis Function

SS Sobol Sequence

SSE Sum of Square Error 


\section{INTRODUÇÃO}

A grande variabilidade nos problemas de Engenharia tem levado muitos pesquisadores a analisar o comportamento de fenômenos em diversos tipos de elementos estruturais. Além disso, os modelos físicos vêm se tornando mais realistas e complexos, sendo, portanto, as incertezas importantes ao passar dos anos para análise dos fenômenos de estudo (Wan et al., 2014; Kroetz, 2015; García et al., 2016). Desta forma, torna-se de fundamental importância quantificar, analisar e prever as incertezas para obter-se resultados mais realistas.

O desenvolvimento de meta modelos vem se tornando cada vez mais populares (Wendland, 1995; Wu, 1995; Bayramov et al., 2004; Liu et al., 2005; Sudret, 2012; Dubourg e Sudret, 2014; Pina et al., 2014) para o problema físico, como por exemplo na predição de incerteza de fenômenos de falha em sistemas estruturais. Assim, o método consiste basicamente na substituição do modelo físico complexo por uma aproximação, denominada meta modelo onde modelos matemáticos mais simples podem ser determinados para a descrição do problema em estudo. Essa metodologia permite também o uso de variáveis aleatórias em simulações, como por exemplo, na simulação de Monte Carlo (Shields et al., 2015; Bierig e Chernov, 2016). Além disso, nesta metodologia, a resposta do sistema estrutural pode ser construída por meio de uma superfície de resposta do tipo $y=g(x)$, onde a partir do vetor de variáveis de entrada $x$, a resposta estrutural $y$ é ajustada por meio de uma regressão, linear ou não linear, validada com a resposta do sistema. Desta forma, o custo computacional é focado na determinação dos parâmetros do meta modelo a partir de observações conhecidas do sistema estrutural em estudo. Um dos métodos mais conhecidos consiste em oferecer um meta modelo polinomial, desde que se conheça os coeficientes do polinômio que são os parâmetros do meta modelo (Myers et al., 2009; Montgomery, 2013; García et al., 2016). Uma segunda alternativa consiste no uso de processos de interpolação, ao qual a partir de um campo amostral, funções de interpolação são utilizadas para cada conjunto de coordenadas do domínio (Wendland, 1995; Liu et al., 2005).

Técnicas de meta modelo geralmente revelam eficiência e acurácia principalmente se o sistema em consideração envolva um número moderado de variáveis (Myers et al., 2009; Sudret, 2012; Montgomery, 2013; García et al., 2016). No entanto, o custo computacional necessário pode aumentar para um número maior de variáveis de entrada. Desta forma, a 
metodologia dos meta modelos exige técnicas de redução de observações do sistema necessárias para o cálculo dos parâmetros do modelo de forma eficiente.

Uma segunda dificuldade relacionada aos meta modelos consiste que os mesmos são determinados através de estimativa de erro por aproximação. Tal avaliação é de importância crucial, já que a precisão dos resultados e a sua acurácia dependerão diretamente do ajuste da superfície de resposta (Myers et al., 2009; Montgomery, 2013). Além disso, uma estimativa de erro permite refinar o modelo até que alguma precisão requerida seja alcançada e, portanto, através desses modelos, reutiliza-los para qualquer valor das variáveis de entrada.

Diante das considerações feitas, pode ser concluída a necessidade de quantificar a incerteza, pois o desenvolvimento de algoritmos, softwares e teorias para quantificar as incertezas e de métodos de confiabilidade nas predições dos comportamentos se destacam como um dos desafios mais importantes da mecânica computacional nos próximos anos. Contudo, no caso desta dissertação, o uso de meta modelos consistirá em solucionar alguns fenômenos selecionados da engenharia, sendo eles relacionados a mecânica da fratura e fadiga, para meta modelagem com superfície de resposta e com interpolação de base radial. Além disso, as amostragens a serem consideradas são relacionadas ao método tradicional de Experimento Fatorial (Santilli et al., 2011; Montgomery, 2013; Tsao e Patel, 2015) e a sequências aleatórias de Hipercubo Latino (Blatman, 2009; Shields e Zhang, 2016) e Sobol (Blatman, 2009; Almeida e Evangelista Jr., 2016).

Para o processo de quantificação da incerteza, diversas publicações têm sido realizadas por Evangelista Jr. e colaboradores, sendo algumas recentes publicações dadas por Almeida e Evangelista Jr. (2016), Borges (2016), García et al. (2016) e Evangelista Jr. e Muños (2014). Dentre estas destacam-se Almeida e Evangelista Jr. (2016) e García et al. (2016) com o uso de meta modelos polinomiais para quantificação da incerteza na predição da vida à fadiga em placas metálicas.

\subsection{MOTIVAÇÃO}

A quantificação da incerteza é um elemento fundamental na Engenharia, sendo a qualidade das decisões tomadas dependentes de uma análise correta da incerteza na saída do modelo. Além disso, a variabilidade, a quantidade e a incerteza dos parâmetros de um modelo para os fenômenos de estudo faz com que os problemas sejam notoriamente complexos. A 
presença de incerteza em modelos matemáticos para análise de fenômenos da Engenharia tem levado muitos pesquisadores a adotar novas soluções.

Como solução alternativa, muitos pesquisadores consideram o uso de meta modelos (Sudret, 2012; Dubourg e Sudret, 2014; Pina et al., 2014). No processo de quantificação da incerteza, meta modelos consistem ainda em um campo recente quando aplicado nos fenômenos da mecânica da fratura e fadiga. Assim, este trabalho visa contribuir para análise destes fenômenos através da quantificação da incerteza dos mesmos.

\subsection{OBJETIVOS}

\subsubsection{Objetivos gerais}

A pesquisa desta dissertação consiste no desenvolvimento de meta modelos probabilísticos para construção da superfície de resposta por meio de funções polinomiais, e principalmente a combinação delas, de base radial, radial base functions $(R B F)$, sendo utilizadas poucas amostras. Estes modelos são aplicados para quantificação da incerteza para problemas de fratura em meios viscoelásticos com envelhecimento e para previsão de vida de fadiga de baixo ciclo.

\subsubsection{Objetivos Específicos}

Os objetivos específicos são:

- Construir meta modelo com um pequeno número de observações. Otimizar o número de amostras ao mínimo possível.

- Estudar o comportamento do ajuste dos meta modelos comparando-os com amostragens de Experimento Fatorial, Hipercubo Latino e Sequência de Sobol.

- Construir meta modelos com o erro de aproximação reduzido. Garantir um grau de precisão adequado ao meta modelo. 


\subsection{CONTRIBUIÇÕES DO TRABALHO}

Para quantificar a incerteza na mecânica da fratura e da fadiga, meta modelos do tipo superfície de resposta é empregado pela literatura principalmente no formato polinomial. Porém, para este trabalho, fora incorporado, além das funções polinomiais, uma nova metodologia para o meta modelo que consiste em um processo de adaptação de funções de base radial para atuar nas superfícies de respostas dos fenômenos. Além disso, é adotado um enriquecimento do meta modelo de $R B F$ com bases polinomiais de primeira ordem, sendo o mesmo, uma inovação no estudo de meta modelos.

\subsection{ESTRUTURA DO TRABALHO}

O trabalho foi estruturado em cinco capítulos. Este capítulo de introdução descreve a importância do tema e a motivação da pesquisa, apresenta também os objetivos, contribuições do trabalho e a organização da dissertação.

O segundo capítulo aborda a fundamentação teórico da dissertação, sendo apresentados fundamentos da quantificação da incerteza e noções sobre meta modelos e das respectivas amostragens adotadas no trabalho.

O terceiro capítulo contém o processo de quantificação da incerteza da vida de fadiga de baixo ciclo em membros estruturais. O capítulo é estruturado em formato de artigo científico, ou seja, com introdução, revisão bibliográfica e análise de resultados.

O quarto capítulo descreve a quantificação da incerteza da taxa de liberação de energia dependente do tempo de um membro estrutural com trinca. Além disso, o capítulo apresenta a mesma estruturação de artigo científico como o terceiro capítulo.

No quinto e último capítulo, são apresentadas as conclusões obtidas no trabalho e as sugestões de trabalhos futuros. 


\section{FUNDAMENTAÇÃO TEÓRICA}

Este capítulo descreve inicialmente conceitos sobre quantificação da incerteza, com destaque às funções densidade de probabilidade e a simulação de Monte Carlo. Além disso, serão abordadas noções sobre meta modelos com o uso de diferentes amostragens como: Experimento Fatorial, Sequência de Sobol e Hipercubo Latino em processos de aproximação. Neste trabalho os processos de aproximação considerados são regressão polinomial e interpolação por funções de base radial.

\subsection{QUANTIFICAÇÃO DA INCERTEZA}

A quantificação da incerteza, como o próprio nome sugere, consiste em uma área dedicada ao estudo de incertezas que podem surgir em fenômenos. O processo de análise de um fenômeno consiste no uso de probabilidade em simulações numéricas (Lopez et al., 2013; Bigoni, 2014; Wan et al., 2014; Hariri-Ardebili e Saouma, 2016; García et al., 2016). Para a predição de incertezas é necessário a presença de um modelo que pode ser definido como uma entidade matemática a qual descreve uma conexão causal entre variáveis de entrada (inputs) e de saída (outputs). Desta forma, os modelos utilizados são de fenômenos da mecânica da fratura e da fadiga.

Na predição, modelos devem ser verificados associando-se condições particulares para os inputs na obtenção dos outputs. Quando se tem modelos complexos, sua validação pode representar um alto custo computacional, com consumo considerável de tempo. Além do mais, é importante determinar corretamente as variáveis e as suas distribuições de probabilidade, pois, as mesmas exercem influência no fenômeno. As distribuições de probabilidade adotadas para os fenômenos analisados são apresentados na próxima seção. Além disso, será adotado o espaço normal padrão ao qual permite que variáveis de diferentes distribuições permaneçam com o mesmo comportamento de distribuição.

\subsubsection{Distribuição de probabilidade}

O comportamento de uma variável aleatória pode ser descrito através da distribuição probabilística. Apresentam-se nesta seção, as distribuições contínuas do tipo normal e de valor extremo, que serão utilizadas neste trabalho. 


\section{Distribuiç̃ão normal}

Caracteriza-se por ser o tipo de distribuição contínua mais importante em todo o campo da estatística. Apresenta-se na Equação (2.1) como uma curva normal e descreve de maneira aproximada muitos fenômenos que ocorrem na natureza. Desta forma, uma variável aleatória $x$ segue a função densidade de probabilidade, ou probability density function $(P D F)$ dada na pela seguinte equação:

$$
f(x ; \mu, \sigma)=\frac{1}{\sqrt{2 \pi \sigma}} e^{\frac{-1}{2 \sigma^{2}(x-\mu)^{2}}},-\infty<x<\infty
$$

em que $\mu$ é o valor esperado, ou a média, e $\sigma$ é o desvio padrão populacional. $\mathrm{O} \mu$ corresponde a um parâmetro de localização do centro da distribuição, e $\sigma$ correspondente a um parâmetro de forma da distribuição. Pode-se ainda relatar que o desvio padrão corresponde a um ponto com mudança de curvatura da curva da função de probabilidade.

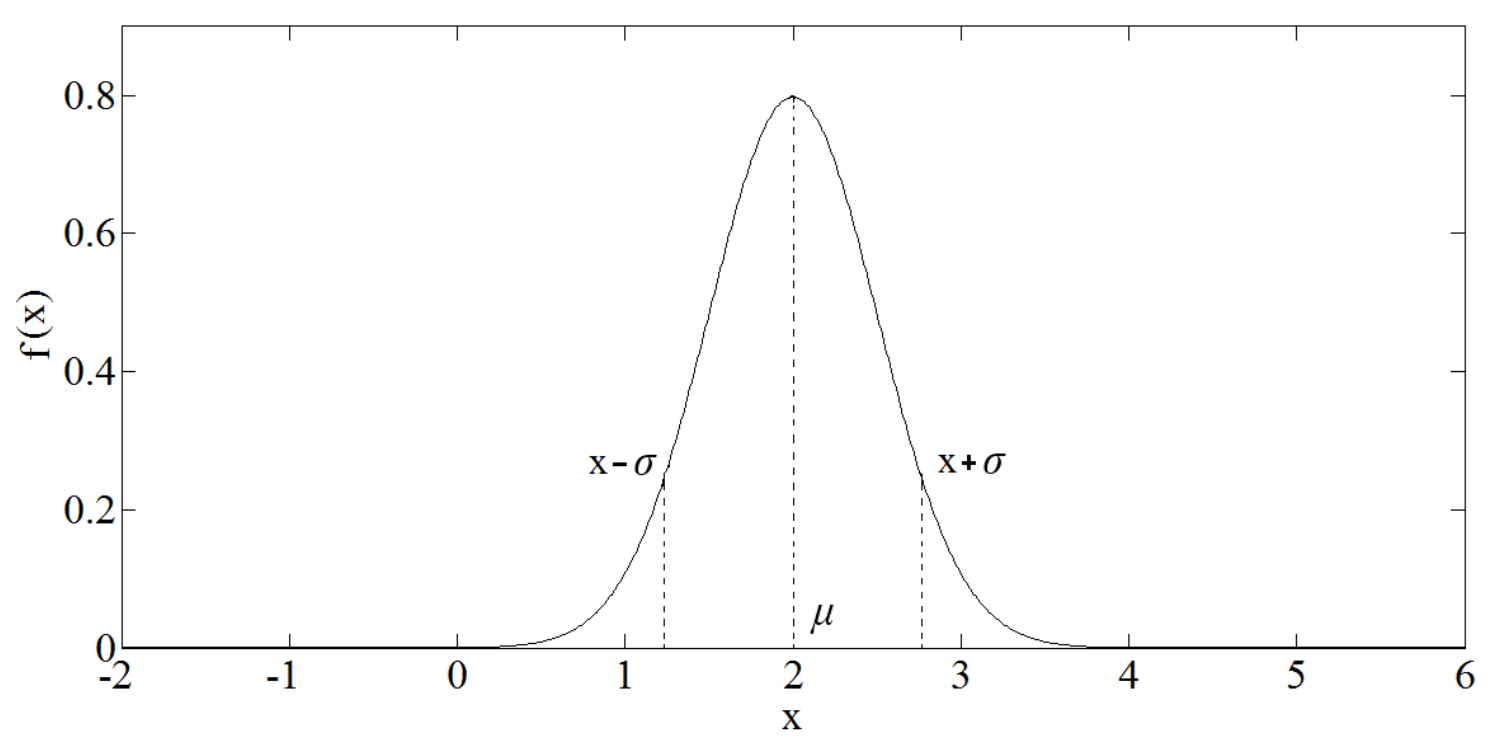

Figura 2.1 - Função densidade de probabilidade (PDF) normal

\section{$\underline{\text { Distribuicãa normal padrão }}$}

A distribuição normal padrão consiste em uma transformação da curva normal para uma distribuição de média nula e desvio padrão unitário. Neste tipo de distribuição é necessário o cálculo de uma coordenada equivalente ( $z$ ). Desta forma, a probabilidade de uma variável $x(\mathrm{~N}(\mu, \sigma))$ é igual a de $z(\mathrm{~N}(0,1))$. 


$$
z=\frac{x-\mu}{\sigma}
$$

\section{Distribuicão de valor extremo generalizado}

A distribuição de valor extremo generalizado, ou Generalized Extreme Value (GEV) consiste em um conjunto de distribuições que dependem principalmente do parâmetro de forma $(\psi)$. Segundo Li et al. (2016) a variação de $\psi$ subdivide a distribuição em classes chamadas de Gumbel, Fréchet e Weibull. Para um valor nulo do fator de forma a distribuição recebe o nome de Gumbel ou GEV Tipo I. Quando o valor de $\psi$ é positivo, denomina-se distribuição Fréchet ou GEV Tipo II. Caso contrário, ou seja, valores de $\psi$ negativos, denomina-se distribuição tipo Weibull ou GEV Tipo III. A Equação (2.3) apresenta a $P D F$ da distribuição.

$$
f\left(x ; \psi, \delta_{o}, q\right)=\frac{1}{\delta} q(x)^{\psi+1} e^{-q(x)}
$$

Em que $\delta_{o}$ é um parâmetro de escala e $q(x)$ um parâmetro da variável $x$ descrito nas Equações (2.4) e (2.5) para diferentes valores de $\psi$. Assim define-se na Figura 2.2 e nas seguintes equações:

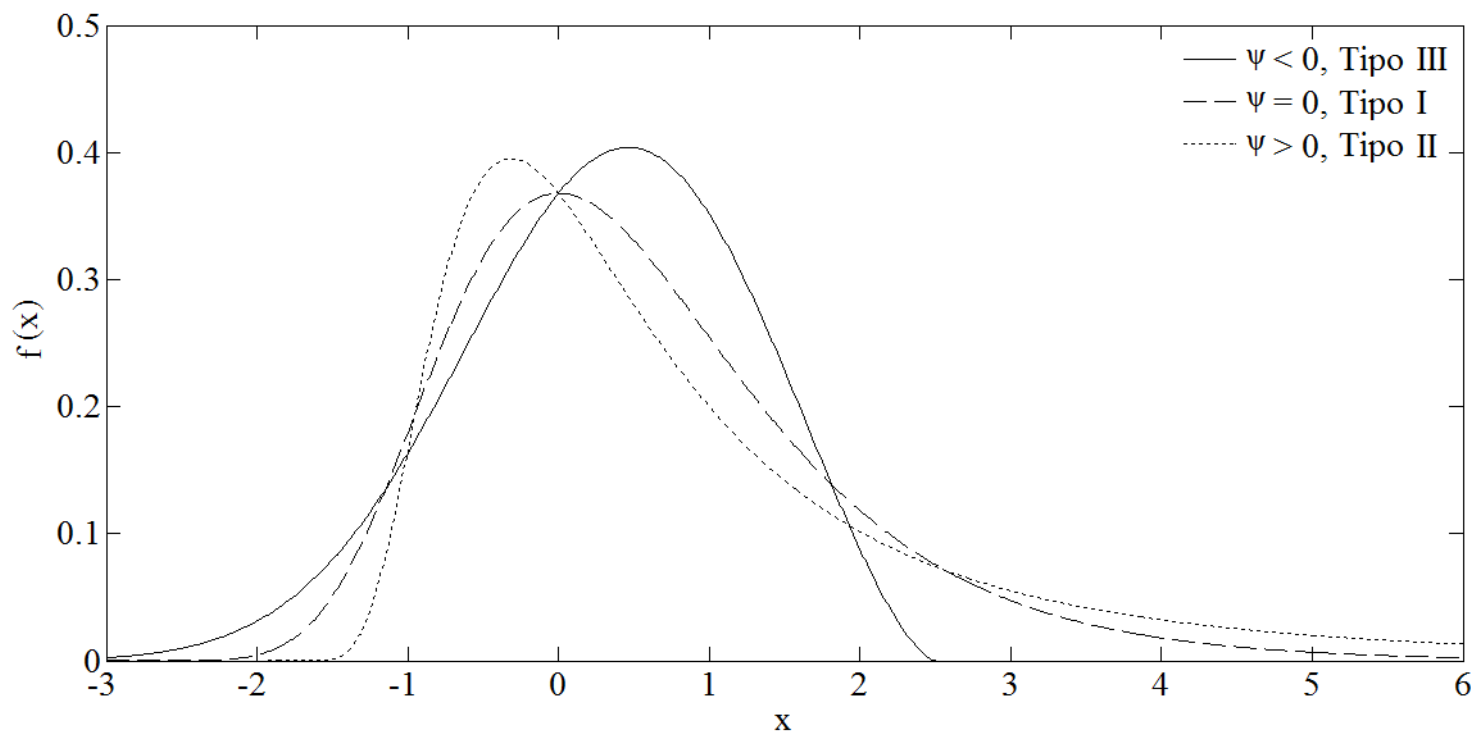

Figura 2.2 - Função densidade de probabilidade $(P D F)$ de $G E V$

$\operatorname{para} \psi=0$ :

$$
q(x)=e^{-\left[-\left(\frac{x-\lambda}{\delta_{o}}\right)\right]}
$$

e para $\psi \neq 0$ : 


$$
q(x)=\left[1+\psi\left(\frac{x-\lambda}{\delta_{o}}\right)\right]^{-1 / \psi}
$$

em que $\lambda$ corresponde ao parâmetro de localização. Na Figura 2.2, observa-se as PDFs em um domínio [-6,3], sendo $\psi<0$ a representação da distribuição Weibull, $\psi=0$ a da Gumbel e $\psi>0$ a de Fréchet.

\subsubsection{Simulação de Monte Carlo}

Uma abordagem direta para quantificar a incerteza pode ser feita pela simulação de Monte Carlo (MC), que é utilizada em quase todos os campos da engenharia e ciência computacional. O método necessita de uma grande quantidade de simulações para que os parâmetros $\mu, \sigma$ e a respectiva $P D F$ sejam estimados através de:

$$
\mu_{y}=\frac{1}{n} \sum_{i=1}^{n} y\left(x_{i}\right)
$$

$\mathrm{e}$

$$
\sigma_{y}^{2}=\frac{1}{n-1} \sum_{i=1}^{n}\left(y\left(x_{i}\right)-\mu_{y}\right)^{2}
$$

em que $\mu_{y}$ corresponde à média da função de saída, $\sigma_{y}{ }^{2}$ a variância do método, $x_{i}$ as variáveis de entrada e $y\left(x_{i}\right)$ a função de saída que modela um fenômeno.

Do ponto de vista estatístico, a simulação por Monte Carlo é determinística desde que o domínio das variáveis de entrada também seja. Além disso, a taxa de convergência dos estimadores baseados no método é lenta e desta forma necessita de muitas simulações para que assim seja alcançada uma boa precisão. Para este trabalho, são utilizadas como técnica de amostragem de entrada o método por Hipercubo Latino, Sequência de Sobol e Experimento Fatorial.

\subsubsection{Geração de números aleatórios}

Uma variável aleatória corresponde à um parâmetro cujo resultado depende de fatores aleatórios. Para geração das variáveis aleatórias, o método da transformação inversa (Figura 2.3) é o mais utilizado, onde se considera $F_{x}\left(x_{i}\right)$ ou $F\left(x_{i}\right)$ como a Função Cumulativa de Probabilidade, ou Cumulative Distribution Function (CDF). Desta forma, por definição, assume-se que $v_{\mathrm{i}}$ é o valor aleatório distribuído uniformemente em [0,1], sendo os valores da variável aleatória o inverso da $C D F$ da Equação (2.8). 


$$
x_{i}=F_{X}^{-1}\left(v_{i}\right)
$$

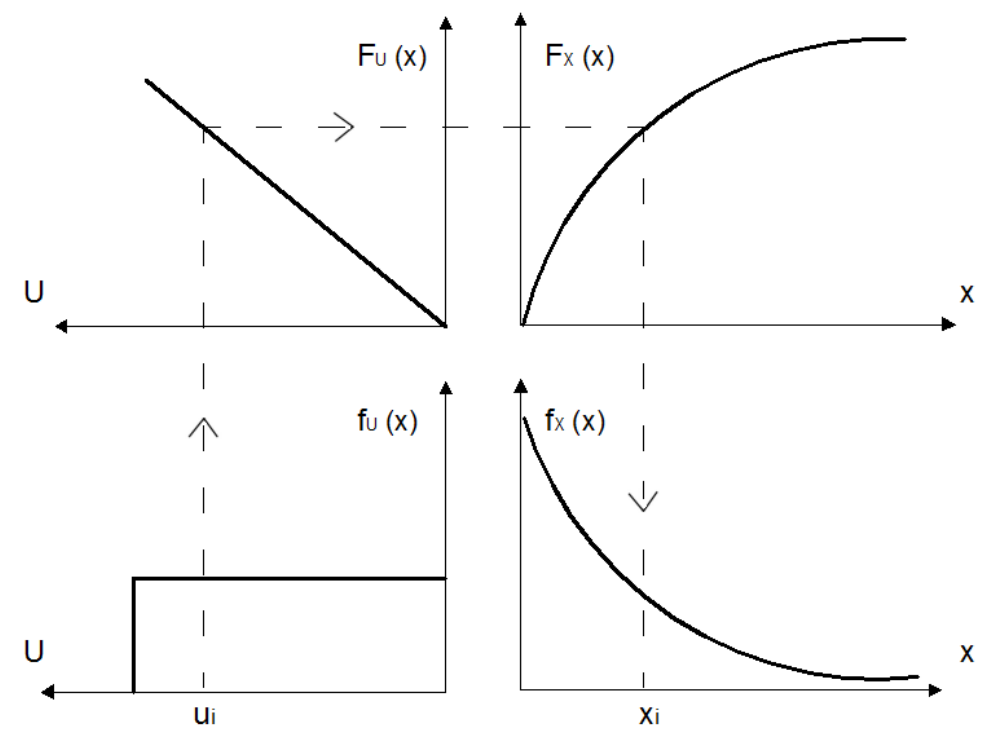

Figura 2.3 - Método Inverso da CDF (Adaptado de: Choi et al., 2007)

Para a Figura 2.3, $u_{i}$ é gerado aleatoriamente na distribuição uniforme entre 0 e 1 de forma a obter a $P D F$ e $C D F$. Além disso, através do inverso de $F_{U}(x)$, se calcula o ponto $x_{i}$ correspondente a essa probabilidade.

\subsubsection{Transformação para espaço normal padrão}

No estudo de qualquer fenômeno é comum o uso de variáveis não normais, como por exemplo, as variáveis do tipo $G E V$ que foram mencionadas anteriormente. No entanto, para obter-se o output ou variável de saída do modelo as mesmas sofrem um processo de padronização que consiste em uma transformação ao espaço normal padrão. O processo segue o modelo de Rosenblatt (1952 apud Borges, 2016) dado a seguir.

$$
\Phi\left(\frac{x_{i}^{*}-\mu_{e q}}{\sigma_{e q}}\right)=F_{X}\left(x_{i}^{*}\right)
$$

Com:

$$
\mu_{e q}=x_{i}^{*}-\Phi\left(F_{X i}\left(x_{i}^{*}\right)\right) \sigma_{e q}
$$

$\mathrm{e}$

$$
\sigma_{e q}=\frac{\phi\left(\Phi^{-1}\left[F_{X i}\left(x_{i}^{*}\right)\right]\right)}{f_{x}\left(x_{i}^{*}\right)}
$$


em que $\Phi$ é a $C D F$ normal padrão, $F_{X}\left(x_{i}{ }^{*}\right)$ a $C D F$ para o ponto de projeto $x_{i}{ }^{*}, \phi$ a $P D F$ normal padrão e $f_{X}\left(x_{i}{ }^{*}\right)$ a $P D F$ para o ponto de projeto $x_{i}{ }^{*}$. Além disso, o processo só pode ser realizado desde que se tenha $F_{X}\left(x_{i}{ }^{*}\right)$. Na Equação (2.9) considera-se que um ponto no espaço de qualquer distribuição $\left(x_{i}\right)$ com a mesma probabilidade normal padrão $\left(z_{i}\right)$ apresenta uma média $\left(\mu_{e q}\right)$ e um desvio $\left(\sigma_{e q}\right)$ equivalente.

\subsection{META MODELOS OU META MODELAGEM}

Meta modelo ou meta modelagem corresponde à análise, construção e desenvolvimento de técnicas, regras, restrições, modelos e teorias aplicáveis ao estudo de um fenômeno. No entanto, nesta dissertação será considerada a construção de um modelo matemático por meio de superfície de resposta que será apresentado posteriormente e será utilizado para regressão polinomial e para interpolação por função de base radial.

\subsubsection{Técnicas de Amostragem}

Nesta seção, são apresentados os parâmetros que serão utilizados no decorrer desta dissertação. É importante salientar que as mesmas consistem em amostragens que podem ser aleatórias ou determinísticas. A amostragem por Experimento Fatorial consiste em uma amostragem determinística tradicional (Santilli et al., 2011; Montgomery, 2013; Tsao e Patel, 2015). Já no caso da amostragem aleatória será considerado Hipercubo Latino e Sequência de Sobol.

\section{Experimento Fatorial}

O método fatorial apresenta duas características importantes que são: os fatores e os níveis. Os fatores adotados na metodologia correspondem às variáveis de entrada (independentes) de um experimento. Já os níveis são valores específicos que são atribuídos ao fator. Neste trabalho um dos fatoriais a ser adotado é o fatorial composto central ou Center Composite Design $(C C D)$ o qual corresponde a uma adaptação do fatorial $2^{k}$, onde há a adição de um ponto central e está representado pelos pontos em negrito na Figura 2.4. 


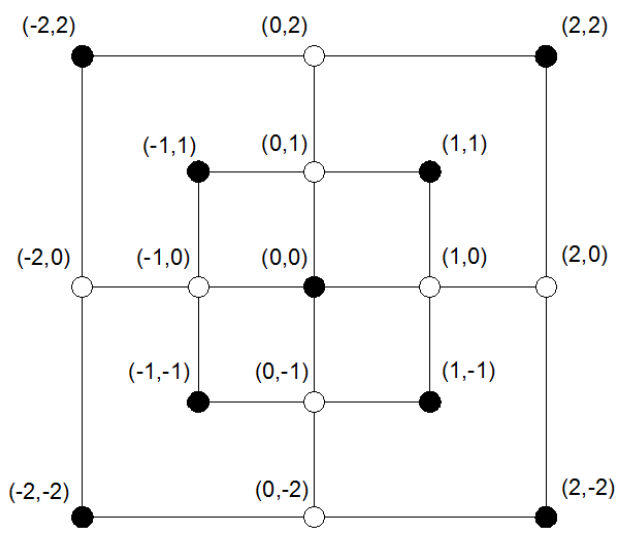

Figura 2.4 - Projeto de Experimento Fatorial

Este tipo de experimento fatorial de nível 2 assume linearidade nos efeitos do fator. No entanto, essa linearidade, segundo Myers et al. (2009), acontece por aproximação e adição de interações entre os fatores. Outro modelo de fatorial que é abordado neste trabalho consiste no fatorial de 3 níveis, ou fatorial completo, ou Full Factorial (FF), quando estes são quantitativos expressam valores mínimo, intermediário e máximo de níveis e são representados por todos os pontos na Figura 2.4. Além disso, pode-se observar na Figura 2.4 que a codificação dos dados para esta pesquisa é $[-2,2]$.

\section{$\underline{\text { Amostragem Hipercubo Latino (LHS) }}$}

Para as variáveis de entrada para uma superfície de resposta, valores aleatórios também podem ser considerados. A amostragem por Hipercubo Latino ou Latin Hypercube Sampling (LHS) é um método para geração de valores aleatórios que utiliza Monte Carlo. No caso de uma função com $n$ variáveis, o intervalo de cada variável é dividido igualmente de acordo com a distribuição que cada variável segue (Figura 2.5).

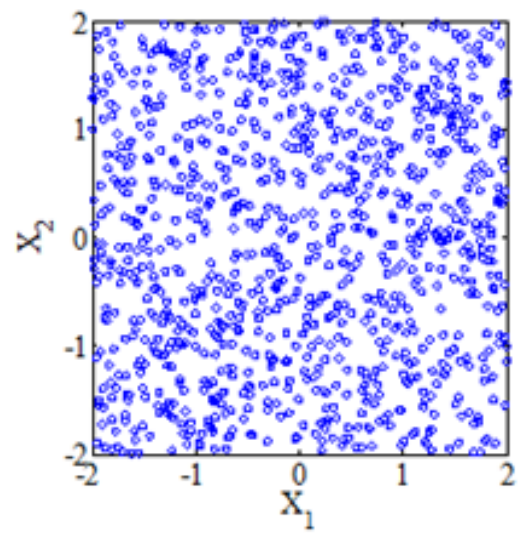

Figura 2.5 - Amostragem Hipercubo Latino 
Uma das principais vantagens do $L H S$ é que o mesmo necessita de menos amostras, diferenciando-se, portanto do Monte Carlo tradicional, que tem uma convergência mais lenta. Desta forma, o número de pontos por variável necessária diminui, de modo que o tempo de processamento se reduz consideravelmente. Pode-se ainda afirmar que esta abordagem assegura que cada uma das variáveis de entrada tem todas as porções da sua gama de valores representados de acordo com a Figura 2.5.

\section{Amostragem Sequência de Sobol (SS)}

Semelhante ao LHS, a Sequência de Sobol ou Sobol Sequence $(S S)$ pode ser responsável por gerar valores aleatórios como variáveis de entrada (Figura 2.6). Pode-se ainda relatar que o mesmo consiste em um exemplo de baixa discrepância quase aleatória e que fora introduzida pelo matemático russo IIya M. Sobol (1967). Esta sequência utiliza uma base igual a dois para formar divisórias sucessivamente mais finas e uniformes do intervalo.

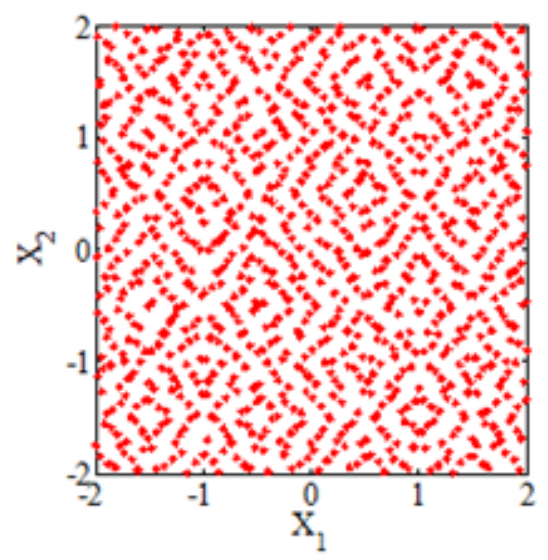

Figura 2.6 - Amostragem Sequência de Sobol

Para o método, a convergência deve ser a mais rápida possível (Almeida e Evangelista Jr., 2016). No entanto, para que isso aconteça, é necessário que os pontos gerados preencham de forma homogênea o domínio de acordo com a Figura 2.6.

\subsubsection{Superfície de Resposta}

A metodologia de superfície de resposta (MSR) é o tipo mais comum de meta modelo e corresponde a um conjunto de técnicas estatísticas e matemáticas usadas no desenvolvimento de modelos matemáticos que descrevem um fenômeno. No problema a ser analisado as variáveis de entrada são chamadas de variáveis independentes e são 
determinadas em ensaios experimentais. Já o desempenho e a qualidade do produto são chamados de resposta ou de variáveis dependentes. As funções de aproximação adotadas pela metodologia consideram o erro estatístico, geralmente assumindo uma distribuição normal de média dos erros residuais igual à zero de acordo com a Equação (2.12).

$$
y=g\left(X_{1}, X_{2}, \ldots, X_{m}\right)+e_{r}
$$

Onde $y$ corresponde a variável dependente; $e_{r}$ ao erro residual e $X_{1}, X_{2}, \ldots X_{m}$ as variáveis independentes ou variáveis naturais. Em muitos casos, é conveniente trabalhar as equações de MSR em variáveis codificadas $\left(\xi_{1}, \xi_{2}, \ldots \xi_{m}\right)$. As variáveis codificadas correspondem a uma transformação das variáveis naturais, representado frequentemente, em valores entre 1 e 1. Além disso, o método de MSR será adotado para regressão polinomial e para funções de base radial.

\section{$\underline{\text { Regressão polinomial com mínimos quadrados }}$}

O método dos mínimos quadrados consiste em estimar coeficientes de uma regressão polinomial. Geralmente polinômios de menor ordem são utilizados como, por exemplo, de primeira e segunda ordem. No uso de polinômios de primeira ordem apresentado na Equação (2.13), estabelece-se que o tamanho da amostra $n$ é maior que o número total das variáveis de entrada $m$, sendo $\xi_{i j}$ correspondente a cada amostra ou nível da variável codificada $\xi_{j}$. Já a Equação (2.14) apresenta-se um polinômio de segunda ordem.

$$
\begin{aligned}
& y_{i}=b_{0}+\sum_{j=1}^{m} b_{j} \xi_{i j}+e_{r} \\
& y_{i}=b_{0}+\sum_{j=1}^{m} b_{j} \xi_{j}+\sum_{j=1}^{m} b_{j j} \xi_{j}^{2}+\sum \sum_{i<j=2}^{m} b_{i j} \xi_{i} \xi_{j}+e_{r}
\end{aligned}
$$

No processo de regressão do polinômio, um dos parâmetros utilizados na superfície de resposta, consiste na minimização do somatório de erros residuais (SSE) dado pela Equação (2.15). Nas Equações (2.16) e (2.17) se apresenta a forma matricial do polinômio de primeira ordem, sendo dado em função de uma matriz modelo $\boldsymbol{A}_{\boldsymbol{o}}$.

$$
S S E=\sum_{i=1}^{n} e_{r}^{2}=\sum_{i=1}^{n}\left(y_{i}-b_{0}-\sum_{j=1}^{m} b_{j} \xi_{i j}\right)^{2}=0
$$




$$
\boldsymbol{y}=\mathbf{A}_{o} \boldsymbol{b}+\boldsymbol{e}_{r}
$$

onde:

$$
\boldsymbol{y}=\left[\begin{array}{c}
y_{1} \\
y_{2} \\
\vdots \\
y_{n}
\end{array}\right], \mathbf{A}_{\boldsymbol{o}}=\left[\begin{array}{ccccc}
1 & \xi_{11} & \xi_{12} & \cdots & \xi_{1 m} \\
1 & \xi_{21} & \xi_{22} & \cdots & \xi_{2 m} \\
\vdots & \vdots & \vdots & & \vdots \\
1 & \xi_{n 1} & \xi_{n 2} & \cdots & \xi_{n m}
\end{array}\right], \boldsymbol{b}=\left[\begin{array}{c}
b_{0} \\
b_{1} \\
\vdots \\
b_{m}
\end{array}\right], \boldsymbol{e}_{r}=\left[\begin{array}{c}
e_{1} \\
e_{2} \\
\vdots \\
e_{n}
\end{array}\right]
$$

De acordo com a representação matricial da equação de regressão, $\boldsymbol{y}$ representa um vetor de $n \times 1$ e $\boldsymbol{A}_{\boldsymbol{o}}$ representa uma matriz $n x s$, onde $s=m+1$. O vetor $\boldsymbol{b}$ representa os coeficientes da regressão sendo da ordem $s \times 1$. O $\boldsymbol{e}_{\boldsymbol{r}}$ corresponde ao vetor de erros aleatórios de $n \times 1$. Além disso, a superfície de resposta de uma função polinomial é representada na Figura 2.7.

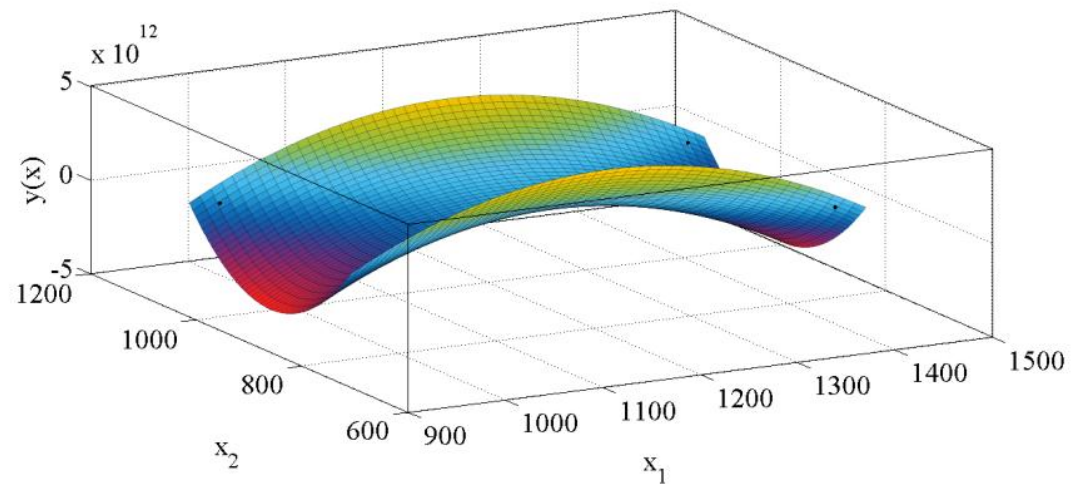

Figura 2.7 - Exemplo de superfície de resposta polinomial

Na Figura 2.7 a superfície de resposta é dada para uma regressão polinomial de segunda ordem, sendo consideradas apenas duas variáveis de entrada $x_{1}$ e $x_{2}$ com uma função de aproximação $y(x)$. Nota-se também que neste caso, a superfície apresenta-se com uma curvatura acentuada a qual é característica de uma função quadrática. Além disso, o processo de regressão é realizado em formato codificado, sendo posteriormente transformado ao espaço normal das variáveis.

A regressão de segunda ordem e de ordens superiores apresenta parâmetros semelhantes à regressão linear. Assim, constata-se que o modelo em formato matricial também é semelhante ao apresentado, havendo apenas, a necessidade de expansão das matrizes. 
Outro parâmetro de análise dos polinômios consiste no erro médio quadrático ou root mean square error (RMSE) dado a seguir.

$$
R M S E=\sqrt{\frac{S S E}{n}}
$$

Na Equação (2.18), nota-se o RMSE, sendo o mesmo responsável por retratar as diferenças entre os valores estimados por uma regressão e os valores da observação. O RMSE serve para agregar as magnitudes dos erros residuais. Por fim, como parâmetro de análise tem-se o coeficiente de determinação $\left(r^{2}\right)$.

$$
\begin{aligned}
& r^{2}=\frac{S_{t}-S S E}{S_{t}} \\
& S_{t}=\sum_{i=1}^{n}\left(y_{i}-\mu_{y}\right)^{2}
\end{aligned}
$$

Para um ajuste perfeito, $S S E=0$ e $r^{2}=1$, ou seja, significa que a reta explica $100 \%$ da variabilidade dos pontos. Entretanto, se $r^{2}=0$ e $S S E=S_{t}$, o ajuste não apresentará nenhuma melhoria. $\mathrm{O} S_{t}$ corresponde à soma dos resíduos totais entre os pontos de entrada e a média dos mesmos. No caso do coeficiente de determinação com um valor próximo de $1 \mathrm{o}$ mesmo não indica que a curva esta realmente ajustada. Alguns pontos que seguem a distribuição não - linear possuem $r^{2}=1$ ou próximo de 1. Uma boa solução para pontos em distribuição linear é plotar e analisar o comportamento de cada um em relação à reta de regressão.

\section{Interpolacão por funcões de base radial (RBF)}

Outro processo de superfície de resposta consiste em adaptar a interpolação por base radial, ou radial basis function $(R B F)$, acrescentando como parâmetros de análise as equações de mínimos quadrados, ou seja, os parâmetros de $S S E, r^{2}$ e RMSE. O processo de interpolação consiste em matrizes que seguem a distância Euclidiana para a função de aproximação. Desta forma, é possível verificar a precisão utilizando dos argumentos de MSR que também são considerados em regressão polinomial por mínimos quadrados. Além disso, apresenta-se a seguir as equações inicias do processo de interpolação. 


$$
\begin{aligned}
& w(\xi)=w(\|\xi\|) \\
& w\left(\xi, r_{o}\right)=w\left(\left\|\xi-r_{o}\right\|\right)
\end{aligned}
$$

Em que || || corresponde a norma Euclidiana. Nas Equações (2.21) e (2.22), define-se $R B F$ como uma função real $w(\xi)$, cujo valor depende apenas da distância a partir da origem ou de uma distancia de um ponto qualquer $r_{o}$, também chamado de ponto central. A soma das $R B F$ é geralmente utilizada para criação de funções de aproximação ou interpolação, sendo neste caso, para modelos de superfície de resposta.

$$
\begin{aligned}
& y=\sum_{j=1}^{n} c_{j} w\left(\left\|\xi-\xi_{j}\right\|\right)+\sum_{j=1}^{m} b_{j} \xi_{j} \\
& {\left[\begin{array}{c}
y_{1} \\
y_{2} \\
\vdots \\
y_{n}
\end{array}\right]=\left[\begin{array}{ccc}
w\left(\left\|\xi_{1}-\xi_{1}\right\|\right) & \cdots & w\left(\left\|\xi_{1}-\xi_{n}\right\|\right) \\
w\left(\left\|\xi_{2}-\xi_{1}\right\|\right) & \cdots & w\left(\left\|\xi_{2}-\xi_{n}\right\|\right) \\
\vdots & & \vdots \\
w\left(\left\|\xi_{n}-\xi_{1}\right\|\right) & \cdots & w\left(\left\|\xi_{n}-\xi_{n}\right\|\right)
\end{array}\right] \cdot\left[\begin{array}{c}
c_{1} \\
c_{2} \\
\vdots \\
c_{n}
\end{array}\right]+\left[\begin{array}{cccc}
1 & \xi_{11} & \cdots & \xi_{1 m} \\
1 & \xi_{21} & \cdots & \xi_{2 m} \\
\vdots & \vdots & & \vdots \\
1 & \xi_{n 1} & \cdots & \xi_{n m}
\end{array}\right] \cdot\left[\begin{array}{c}
b_{1} \\
b_{2} \\
\vdots \\
b_{m}
\end{array}\right]}
\end{aligned}
$$

De acordo com Regis (2016), a Equação (2.23) corresponde à função de aproximação genérica para $R B F$ com presença de coeficientes de um polinômio de primeira ordem, sendo a mesma desenvolvida na Equação (2.24). Entretanto, é importante relatar que existem diversas funções de base radial, sendo para este trabalho, adotado a de $W u-C 2$ na Equação (2.25):

$$
w(r, \rho)=\left(1-\frac{r}{\rho}\right)^{5}\left(8+40 \frac{r}{\rho}+48 \frac{r^{2}}{\rho^{2}}+25 \frac{r^{3}}{\rho^{3}}+5 \frac{r^{4}}{\rho^{4}}\right)
$$

e a de Wendland - C2 apresentada na Equação (2.26).

$$
w(r, \rho)=\left(1-\frac{r}{\rho}\right)^{4}\left(1+4 \frac{r}{\rho}\right)
$$

As Equações (2.25) e (2.26) foram desenvolvidas por Wu (1995) e por Wendland (1995), sendo também chamadas de funções de base radial compactas ou compactly supported radial basis functions (CSRBFs). Além disso, segundo o processo de interpolação, é 
necessário o uso de variáveis codificadas $(\xi)$, sendo $r=\left\|\xi-\xi_{j}\right\|$ e $\rho$ um parâmetro de forma que corresponde ao tamanho local do domínio de atuação de cada ponto central. $\mathrm{O}$ parâmetro $\rho$ depende de um parâmetro de suporte $(\delta)$ e do número de amostras $(n)$ de acordo com a Equação (2.27). Já no caso da parcela polinomial, a mesma pode ser desprezada, caso não se haja necessidade do uso da mesma.

$$
\rho=\left(\frac{\sum_{j=1}^{n} w\left(\left\|\xi-\xi_{j}\right\|\right)}{n-1}\right) \delta
$$

A Figura 2.8 apresenta uma superfície de resposta para $R B F$ do tipo $W u-C 2$ da Equação (2.25) sem o uso da parcela polinomial e para duas variáveis de entrada $x_{1}$ e $x_{2}$.

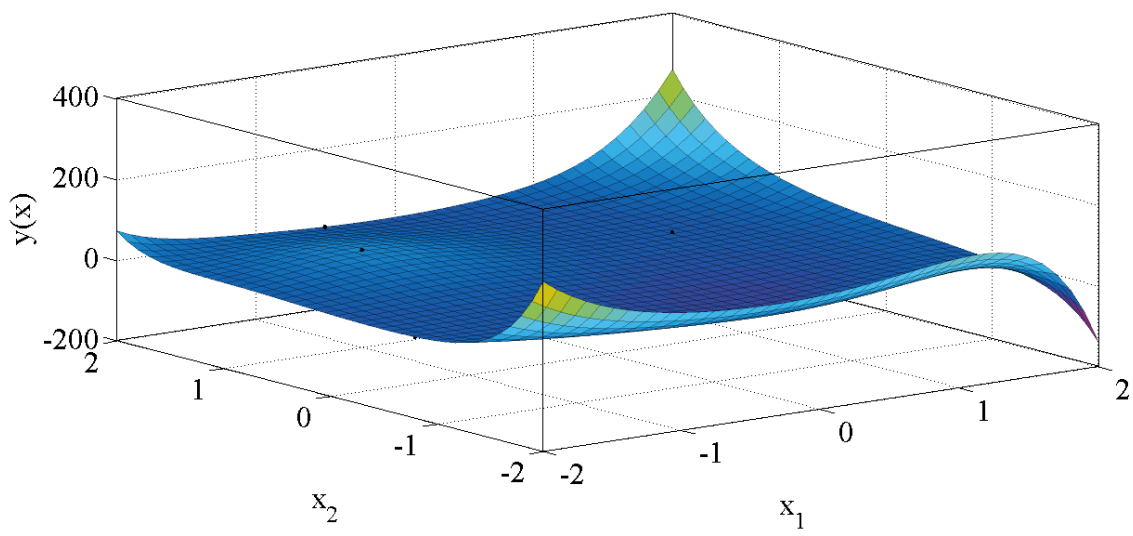

Figura 2.8 - Exemplo Superfície de Resposta para $W u-C 2$

No caso da Figura 2.8, cada ponto amostral apresenta uma $R B F$ conectada a um ponto central que neste caso são os próprios inputs, diferenciando-se, portanto, do método de regressão polinomial. Além disso, o processo deve ser realizado no espaço codificado e posteriormente o mesmo deve retornar ao espaço natural. Por fim, os coeficientes $\left(c_{1}, c_{2}\right.$, $\ldots, c_{n}$ ) originados pela interpolação geralmente se assemelham ao processo dos mínimos quadrados e estão sempre relacionados aos pontos centrais. Desta forma, é necessário um número de pontos centrais mínimo possível de forma a não prejudicar o processo de interpolação. Já para Wendland $-C 2$ apresenta-se a Figura 2.9 com duas variáveis $x_{1}$ e $x_{2}$. 


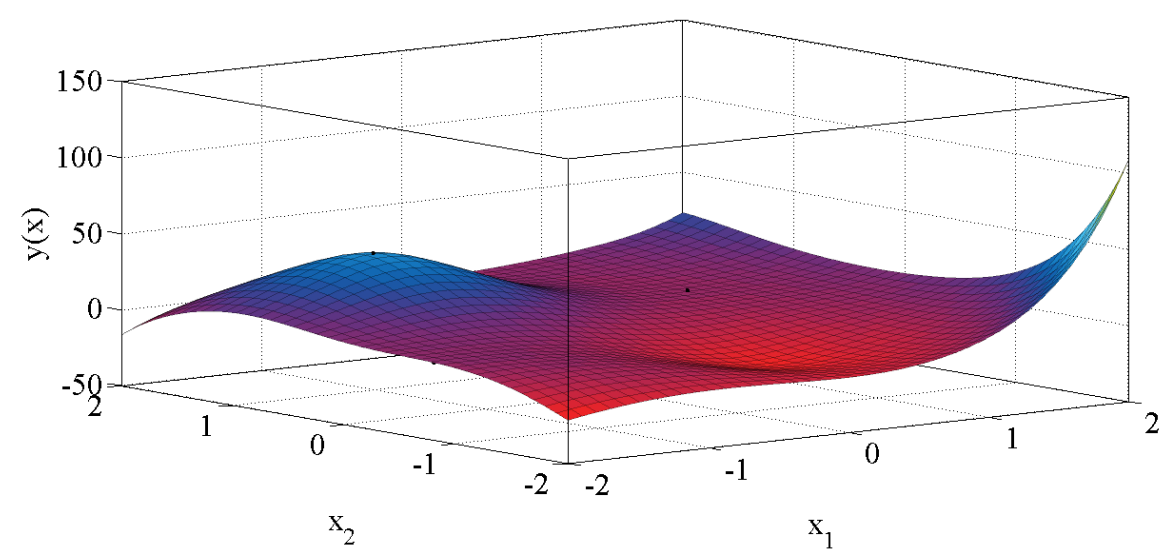

Figura 2.9 - Exemplo de resposta para Wendland - C2

A Figura 2.9 também foi construída para uma amostra bidimensional, onde cada ponta da amostra tende a apresentar o mesmo comportamento de $W u-C 2$. 


\section{QUANTIFICAÇÃO DA INCERTEZA DA VIDA DE FADIGA DE BAIXO CICLO EM MEMBROS ESTRUTURAIS}

\subsection{INTRODUÇÃO}

Nesta seção apresentam-se trabalhos publicados com meta modelos para análise de fenômenos da mecânica de fadiga na literatura atual, sendo os Fang et al. (2014), Xia et al. (2015), Aalae et al. (2016), García et al. (2016), Zhao et al. (2016) e Li et al. (2017) alguns trabalhos publicados com meta modelos. Além disso, é importante salientar que os trabalhos publicados apresentam como meta modelos superfícies de resposta polinomiais e interpolações por funções de base radial.

$\mathrm{Na}$ análise de meta modelos com superfície de resposta polinomial destacam-se os de Xia et al. (2015), Fang et al. (2014), García et al. (2016). No caso de Xia et al. (2015) são considerados que as condições de contorno da vida de fadiga para uma conexão metálica parafusada podem ser determinadas com o uso de meta modelo polinomiais. Outra pesquisa de grande interesse é a de Fang et al. (2014) que considera meta modelos tanto para superfícies polinomiais quanto para interpolação de funções de base radial. De acordo com Fang et al. (2014), o objetivo é otimizar a análise de fadiga em cabines de caminhões através do uso de superfícies de resposta e assim maximizar a vida útil das mesmas. Por fim, García et al. (2016) analisa meta modelos com funções polinomiais de expansão de Chaos em placas metálicas solicitadas a fadiga. Além disso, outro tipo de metodologia bastante usado consiste nas funções de base radial, sendo Wahid e Ahmad (2014), Cao et al. (2015), Wang et al. (2016) algumas publicações recentes.

O uso de funções de base radial é bem comum em meta modelos para análise em fadiga destacando-se publicações de Aalae et al. (2016), Zhao et al. (2016), Li et al. (2017). No caso de Aalae et al. (2016), realizou-se uma análise de um eixo de suspensão de um automóvel através de meta modelos com funções de base radial. Para Aalae et al. (2016), o uso deste tipo de meta modelos apresenta bons resultados permitindo o surgimento de uma nova concepção para análise da resistência dos eixos de suspensão. Por fim, sobressaem-se as publicações de Zhao et al. (2016) e Li et al. (2017). Para Zhao et al. (2016), o uso de meta modelos acontece através de funções de base em redes neurais artificiais para análise de parâmetros de carregamento cíclico em liga de titânio Ti-6AL-4V, apresentando resultados coerentes aos dados experimentais. Já para Li et al. (2017), os meta modelos são 
utilizados para análise de propriedades de fadiga em materiais. Desta forma, percebe-se que o uso de meta modelos é constantemente empregado em diversas áreas da engenharia.

Para este trabalho o uso de meta modelos é realizado através de amostragens tradicionais em conjunto a amostragens aleatórias. Além disso, percebe-se que ainda há uma necessidade do estudo do método em fadiga, portanto, o presente trabalho torna-se de grande importância.

\subsection{ESTUDO DE CASO EM PLACA METÁLICA}

\subsubsection{Parâmetros do Modelo}

O problema a ser analisado consiste em um membro estrutural representado por uma placa metálica com um furo central, sendo $W$ sua largura, $R$ o raio do furo. Na Figura 3.1, apresenta-se a placa que consiste em uma liga de alumínio 2024-T351.

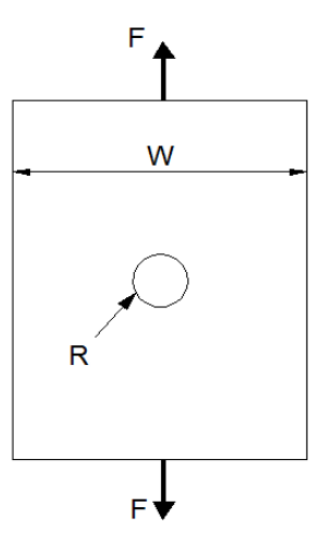

(a) Placa com furo

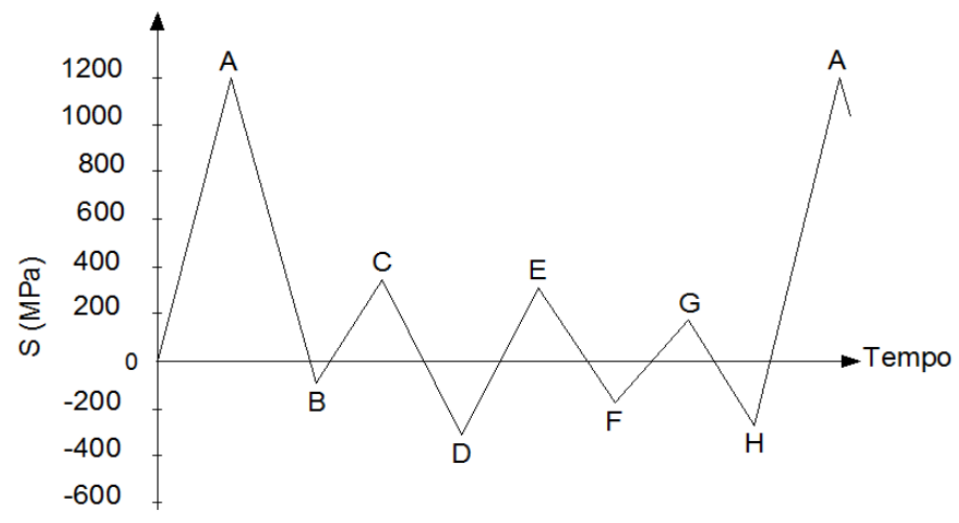

(b) História de tensão nominal aplicada

Figura 3.1 - Placa com furo sob força axial (a) e história de tensão aplicada (b)

A Figura 3.1a ilustra a geometria da placa e o carregamento aplicado $(F)$, enquanto a Figura 3.1b apresenta a história de tensão nominal induzida em $M P a$.

\section{Fadiga em placa com entalhe}

Frequentemente componentes estruturais são submetidos a carregamentos repetitivos, resultando estes, em tensões cíclicas as quais podem gerar danos físicos microscópicos aos materiais envolvidos e eventualmente danos macroscópicos. Assim, de acordo com Dowling (2013) para qualquer tensão menor que a tensão última do material, esse dano pode se acumular e com aplicações cíclicas dessa tensão, pode-se surgir macro trincas que 
levarão a falha do material. Este processo recebe o nome de fadiga e consiste em uma grande área de estudo da engenharia.

A estimativa da vida de fadiga de baixo ciclo de componentes entalhados pela metodologia deformação-vida necessita das tensões e deformações atuantes na raiz do entalhe. Definese fadiga de baixo ciclo quando a falha do material acontece com um número baixo de ciclos. Segundo Agrawal et al. (2014), a mesma ocorre na presença de tensão e deformação predominantemente plástica, com fratura a menos de $10^{5}$ ciclos geralmente. Estas tensões e deformações podem ser determinadas via elementos finitos e dependendo do problema, demandam um tempo de processamento elevado. Como solução do problema, métodos de aproximação podem ser utilizados, como por exemplo, o método de Neuber (1961, apud Negrão, 2014) adaptado para atender a carregamentos cíclicos por meio das equações a seguir:

$$
\Delta \varepsilon=\frac{\Delta \sigma}{E_{m}}+2\left(\frac{\Delta \sigma}{2 H^{\prime}}\right)^{\frac{1}{n^{\prime}}}
$$

com

$$
\Delta \sigma \Delta \varepsilon=\frac{\left(K_{f} \Delta S\right)^{2}}{E_{m}}
$$

e

$$
K_{f}=\sqrt{K_{\sigma} K_{\varepsilon}}=\sqrt{\left(\frac{\Delta \sigma}{\Delta S}\right)\left(\frac{\Delta \varepsilon}{\Delta e}\right)}
$$

em que $K_{f}$ (adimensional) é o fator de concentração de tensão à fadiga, $K_{\varepsilon}$ (adimensional) é o fator de concentração de deformação, $K_{\sigma}$ (adimensional) é o fator de concentração de tensão, $\Delta \sigma$ a variação de tensão, $\Delta \varepsilon$ consiste na variação de deformação na raiz do entalhe, $\Delta S$ a variação da tensão nominal, $\Delta e$ a variação da deformação nominal, $H$ ' o coeficiente de encruamento cíclico, $n$ 'é o expoente de encruamento cíclico, e $E_{m}$ corresponde ao módulo de elasticidade do material. Já para verificação do número de ciclos de vida à fadiga $\left(N_{f}\right)$, o modelo de Morrow (1968 apud Negrão, 2014) é um dos mais adotados na literatura por meio de processos interativos o efeito da tensão média é determinado. 


$$
2 N_{f}=\left[\frac{\varepsilon_{a}-\left(\frac{\sigma_{f}^{\prime}-\sigma_{m}}{E_{m}}\right)\left(2 N_{f}\right)^{b_{k}}}{\varepsilon_{f}^{\prime}}\right]^{\frac{1}{c_{k}}}
$$

A Equação (3.4) descreve o modelo de forma simplificada, sendo $\sigma_{m}$ a tensão média que é calculada pelas tensões $\Delta \sigma, \sigma_{f}$ ' o coeficiente de resistência a fadiga cíclica, $\varepsilon_{f}^{\prime}$ o coeficiente de ductilidade a fadiga, $\varepsilon_{a}$ a amplitude de deformação total e $b_{k}$ e $c_{k}$ são coeficientes de uma regressão na escala logarítmica de $x$ e y.

\subsubsection{Construção dos meta modelos}

Esta subseção dedica-se à metodologia dos meta modelos para estimativa da vida de fadiga de baixo ciclo de um membro estrutural. No caso da metodologia, a Figura 3.2 apresenta um fluxograma da construção e determinação do meta modelo.

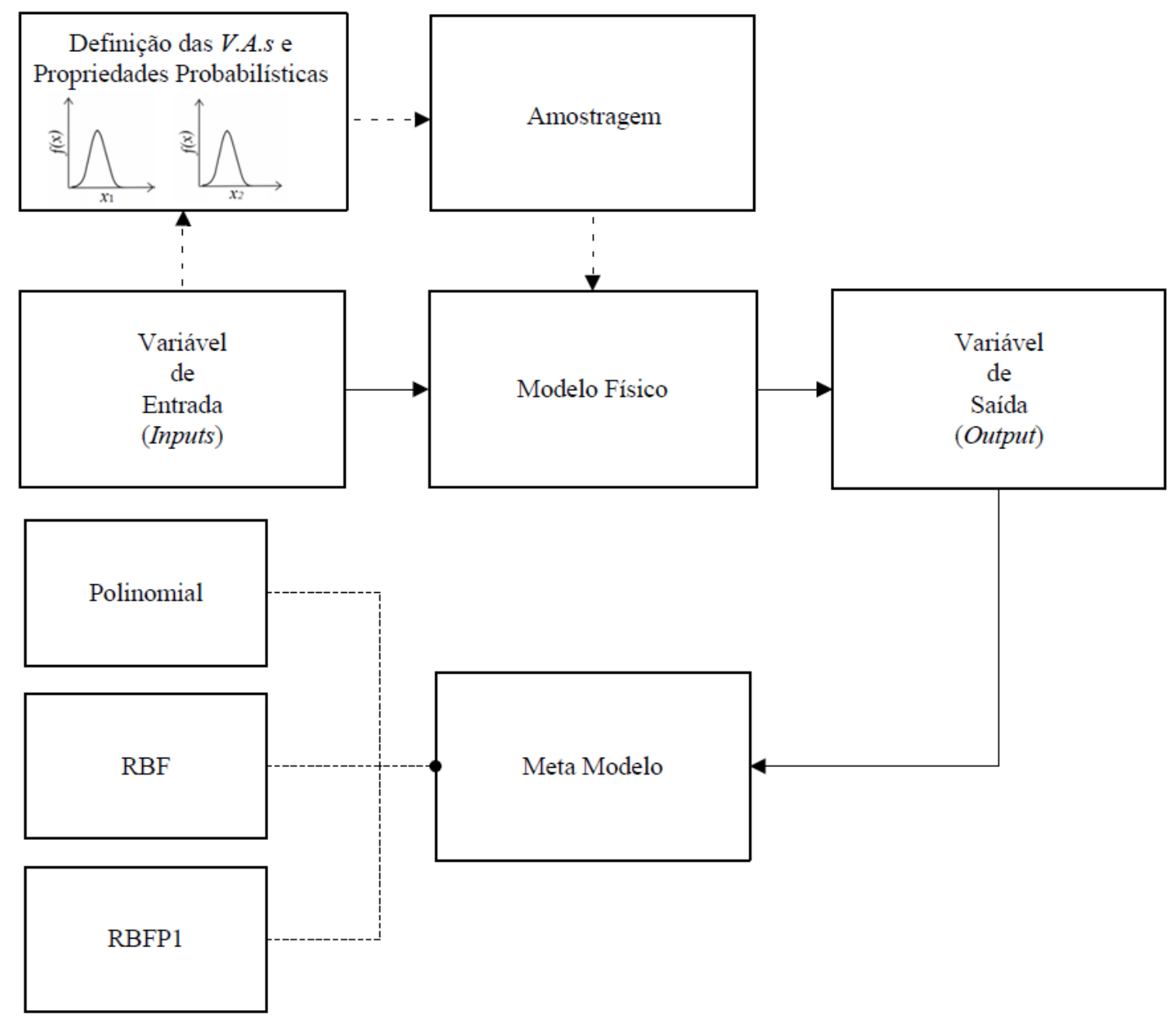

Figura 3.2 - Fluxograma para construção do meta modelo 
Nota-se na Figura 3.2 que, para a construção e determinação dos coeficientes dos meta modelos, tem-se a necessidade da entrada de parâmetros iniciais e de amostragens para o modelo físico de qualquer fenômeno. Para um meta modelo determinístico, utiliza-se o processo apresentado na Figura 3.2 com linhas contínuas. Porém, se o meta modelo apresentar parâmetros aleatórias é necessário à determinação das variáveis aleatórias (V.A.s) e suas respectivas propriedades probabilísticas. Assim, pode-se então determinar as amostragens aleatórias para alimentar o meta modelo. Além disso, as superfícies de respostas podem ser construídas através de uma regressão polinomial $P 2$, ou por processos de interpolação por $R B F$ ou $R B F P 1$. Por fim, determina-se os coeficientes do meta modelo para a superfície de resposta que está sendo incorporada ao modelo. Na Figura 3.3 apresenta-se o processo para quantificação da incerteza.

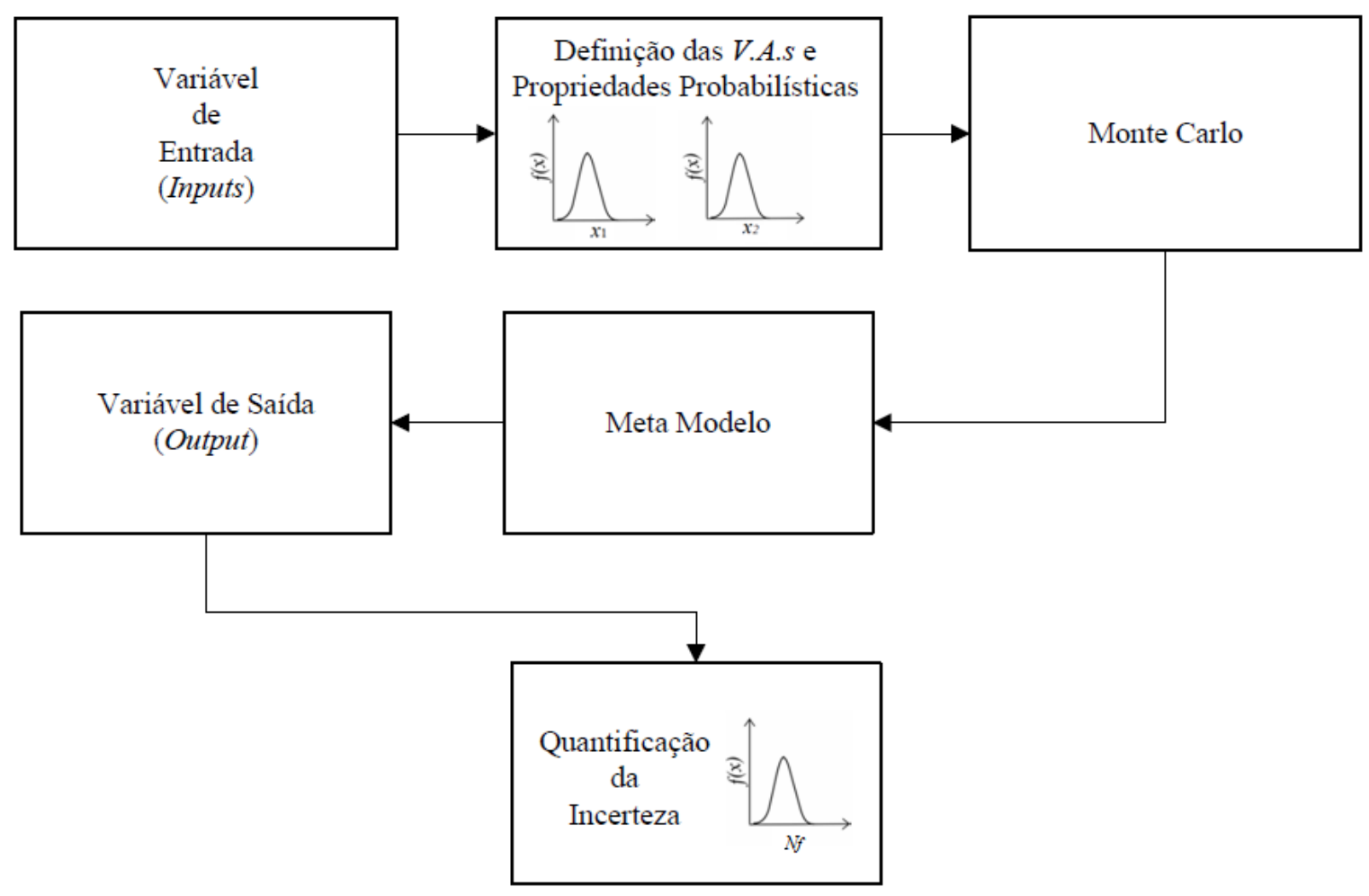

Figura 3.3 - Fluxograma para quantificação da incerteza em fadiga

Na Figura 3.3, novos dados de entrada são considerados e incorporados ao meta modelo. Para o Monte Carlo, 5 E+5 simulações são introduzidas ao meta modelo. Por fim, com a saída do meta modelo, o processo de quantificação é realizado. Para os parâmetros do sistema ou Inputs consideram-se as variáveis determinísticas da Tabela 3.1. 
Tabela 3.1 - Valor das variáveis determinísticas à fadiga

\begin{tabular}{c|c|c}
\hline Var & Unid & Valor \\
\hline$E$ & $M P a$ & 310 \\
\hline$F$ & $M P a$ & -172 \\
\hline$G$ & $M P a$ & 172 \\
\hline$H$ & $M P a$ & -241 \\
\hline$E_{m}$ & $M P a$ & 73100 \\
\hline$b_{k}$ & - & $-0,113$ \\
\hline$c_{k}$ & - & $-0,713$ \\
\hline$K_{f}$ & - & 2,4 \\
\hline-$)$ & \multicolumn{2}{|c}{ adimensional }
\end{tabular}

Na Tabela 3.1, as variáveis $E, F, G$ e $H$ correspondem à parcela do histórico de tensões e as variáveis $b_{k}$ e $c_{k}$, sendo estas determinadas por regressão log-log. Além disso, na Tabela 3.2 observam-se as variáveis aleatórias, sendo as mesmas adotadas como variáveis de entrada.

Tabela 3.2 - Valor das variáveis aleatórias à fadiga

\begin{tabular}{c|c|c|c|c|c|c}
\hline Parâmetro & Variável & Unid & $\mu$ & $\sigma$ & $C V$ & $f(x)$ \\
\hline \multirow{4}{*}{ Carregamento } & $A$ & $M P a$ & 1200 & 240 & $20 \%$ & Normal \\
\cline { 2 - 7 } & $B$ & $M P a$ & -69 & 13,8 & $20 \%$ & Normal \\
\cline { 2 - 7 } & $C$ & $M P a$ & 345 & 69 & $20 \%$ & Normal \\
\cline { 2 - 7 } Material & $D$ & $M P a$ & -310 & 62 & $20 \%$ & Normal \\
\hline & $H^{\prime}$ & - & 662 & 99,3 & $15 \%$ & Normal \\
\cline { 2 - 7 } & $n^{\prime}$ & - & 0,07 & 0,0105 & $15 \%$ & Normal \\
\cline { 2 - 7 } & $\sigma_{f^{\prime}}^{\prime}$ & $M P a$ & 927 & 139,05 & $15 \%$ & Normal \\
\hline$(-)$ & $\varepsilon_{f}^{\prime}$ & - & 0,409 & 0,0613 & $15 \%$ & Normal \\
\hline
\end{tabular}

Na Tabela 3.2 nota-se que as tensões $A, B, C, D$ são dadas em $M P a$ e seguem uma distribuição normal. Os parâmetros do material $H^{\prime}, n^{\prime}, \sigma_{f}^{\prime} e$ ef também atendem a uma distribuição normal. Além disso, se apresenta $\mu, \sigma$ e CV para as variáveis, sendo CV o coeficiente de variação das variáveis.

Para a regressão polinomial $P 2$ considerou-se $F F, C C D, L H S$ e $S S$. No caso do $F F$, foram adotados amostras a um e dois desvios padrões $\left(F F^{I / I}\right)$ e outro modelo a dois desvios padrões $\left(F F^{\mathrm{II}}\right)$ tendo, portanto 13121 e 6561 pontos por variável de entrada respectivamente. No entanto para o $C C D$, o número é inferior, tendo apenas 513 pontos $\left(C C D^{I / I I}\right)$ e 257 pontos $\left(C C D^{I I}\right)$ por variável de entrada sendo semelhante ao $F F$. Já na Figura 3.4, apresentam-se as amostragens utilizadas neste trabalho. 


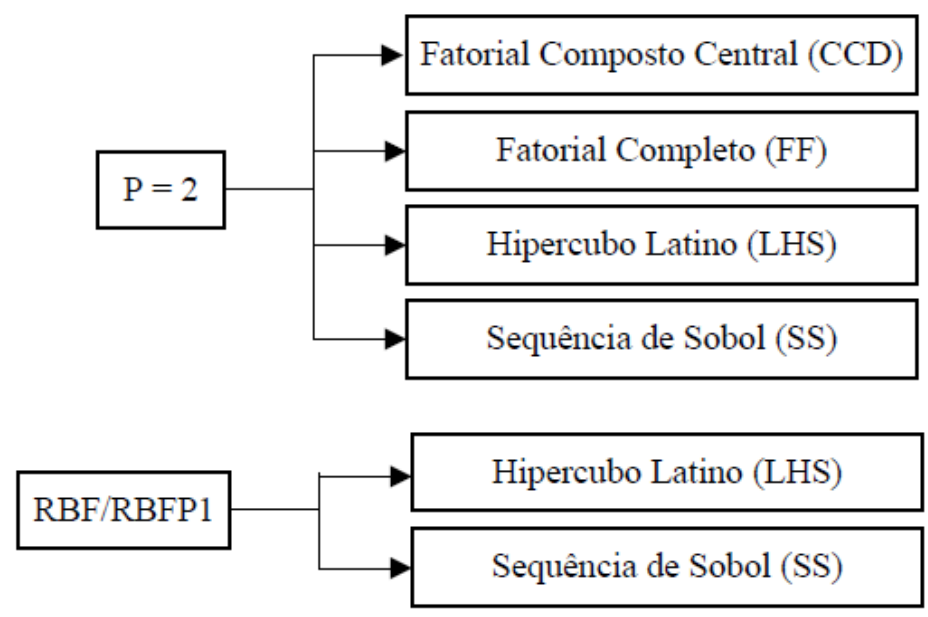

Figura 3.4 - Modelos de amostragens

Para $P 2$, consideraram-se inicialmente valores aleatórios de $S S^{I I}$ e de $L H S^{I I}$, sendo os mesmos representados por um domínio de [-2,2] desvios padrões com 125 amostras. Além disso, fora realizada uma análise comparativa dos valores de $L H S$ e $S S$ para 2, 3 e 4 desvios padrões com 125 amostras por superfície de resposta. Em contra partida, para $R B F$ e $R B F P 1$, a amostragem considerada fora apenas para dois e três desvios padrões de $L H S$ e SS. Por fim, para o número de ciclos de vida, adotou-se a metodologia de Morrow (1968) através de uma rotina de Negrão (2014).

\subsection{ANÁLISE DE RESULTADOS}

Nesta etapa, apresentam-se os resultados da solicitação a fadiga do membro estrutural para $P 2, R B F$ e $R B F P 1$ para amostragens de $F F, C C D, L H S$ e $S S$. Além disso, para as RBFs e $R B F P 1$ considerou-se o tipo Wendland-C2 e para o suporte $(\delta)$, adotou-se $\delta=1$. Na Tabela 3.3, são apresentados os parâmetros de regressão SSE, RMSE e $r^{2}$ para $P 2$ para dois e um e dois desvios padrões. Além disso, $N$ corresponde ao número de amostras para determinação dos coeficientes do meta modelo.

Tabela 3.3 - Parâmetros da regressão de P2 em $N_{f}$

\begin{tabular}{c|c|c|c|c}
\hline $\begin{array}{c}\text { Amostragem } \\
N_{f}\end{array}$ & $S S E$ & $\mathrm{r}^{2}$ & $R M S E$ & $\mathrm{~N}$ \\
\hline $\mathrm{FF}^{\mathrm{II}}$ & $2,75 \mathrm{E}+4$ & 0,96 & 2,05 & 6561 \\
\hline $\mathrm{FF}^{\mathrm{I} / I}$ & $3,50 \mathrm{E}+4$ & 0,95 & 1,63 & 13121 \\
\hline $\mathrm{CCD}^{\mathrm{II}}$ & $1,34 \mathrm{E}+3$ & 0,97 & 2,28 & 257 \\
\hline $\mathrm{CCD}^{\mathrm{I} / \mathrm{II}}$ & $2,08 \mathrm{E}+3$ & 0,96 & 2,01 & 513 \\
\hline $\mathrm{LHS}^{\mathrm{II}}$ & $1,61 \mathrm{E}+2$ & 0,96 & 1,14 & 125 \\
\hline $\mathrm{SS}^{\mathrm{II}}$ & $5,37 \mathrm{E}+1$ & 0,99 & 0,65 & 125 \\
\hline
\end{tabular}


Na Tabela 3.3 o somatório de erros (SSE) tende a diminuir consideravelmente quando os dados de entrada não seguem o padrão do fatorial. No caso do RMSE, percebe-se que o mesmo se assemelha ao SSE, ou seja, para valores de regressão $L H S$ e SS os mesmos se apresentam inferiores aos dos experimentos fatoriais e, portanto a regressão se torna mais eficiente. Por fim, para o coeficiente de determinação, nota-se que mais de $95 \%$ da variabilidade dos pontos pode ser explicada pela regressão de todos os modelos. Outra análise consiste nas superfícies de resposta apresentadas na Figura 3.5 para $P 2$.

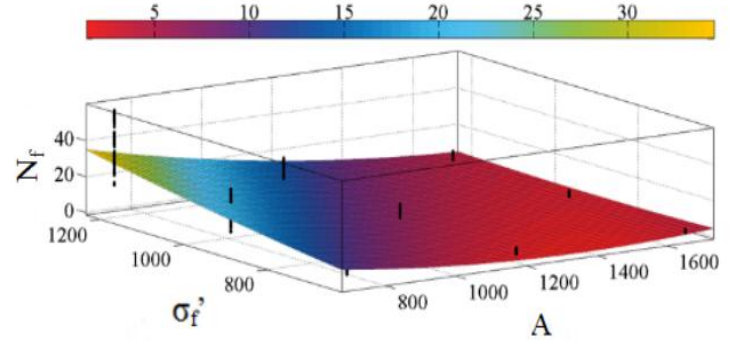

(a) $\mathrm{FF}^{\mathrm{II}}$

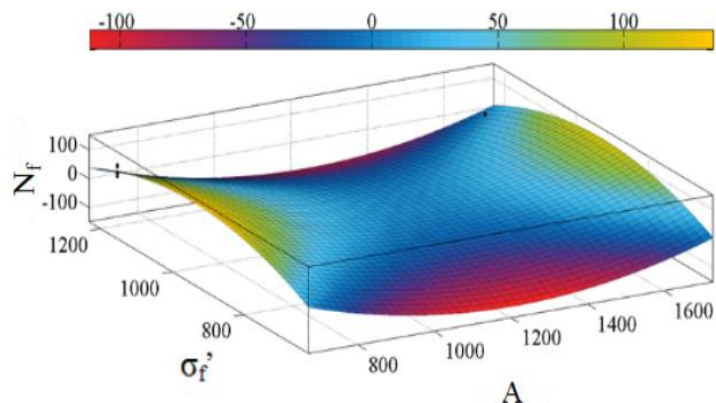

(c) $\mathrm{CCD}^{\mathrm{II}}$

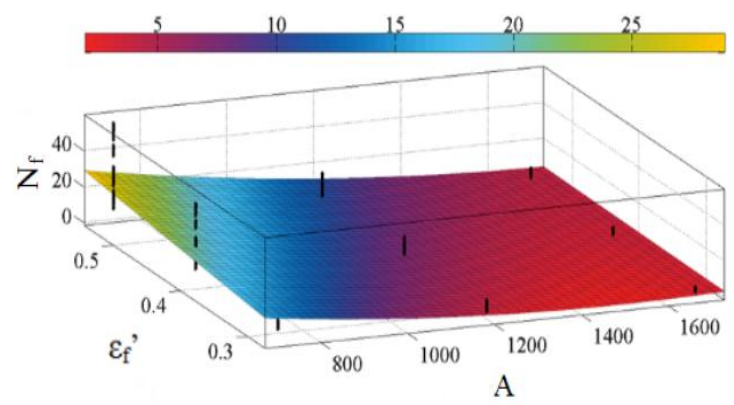

(b) $\mathrm{FF}^{\text {II }}$

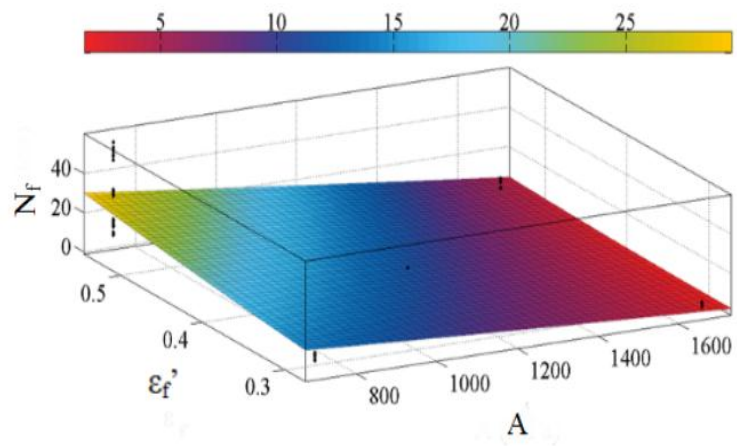

(d) $\mathrm{CCD}^{\mathrm{II}}$

Figura 3.5 - Superfície de Resposta de P2 para (a) e (b) com FF ${ }^{I I}$ e para (c) e (d) com $\mathrm{CCD}^{\mathrm{II}}$

Os resultados da regressão da Tabela 3.3 se apresentam nas Figuras 3.5 a 3.7 e expressam o número de ciclos de vida à fadiga do membro estrutural em função das três principais variáveis aleatórias que são o primeiro vetor de tensão solicitante cíclica $(A)$, o coeficiente de resistência à fadiga cíclica $\left(\sigma_{f}^{\prime}\right)$ e o coeficiente de ductilidade a fadiga $\left(\varepsilon_{f}{ }^{\prime}\right)$. A Figura 3.5a-b corresponde ao $\mathrm{FF}^{\mathrm{II}}$ e a Figura $3.5 \mathrm{c}-\mathrm{d}$ ao $\mathrm{CCD}^{\mathrm{II}}$ e pode-se ainda aferir das mesmas que o fatorial composto central apresenta uma curvatura mais acentuada principalmente para as variáveis aleatórias $A$ e $\sigma_{f}$ '. Além disso, também na mesma figura pode-se observar a existência de uma escala indicando o número de ciclos de vida para cada superfície de resposta que apresenta uma predominância na mesma entre 5 e 15 ciclos de vida à fadiga. Outra análise consiste no FF e CCD a 1 e 2 desvios padrões dado pela Figura 3.6. 


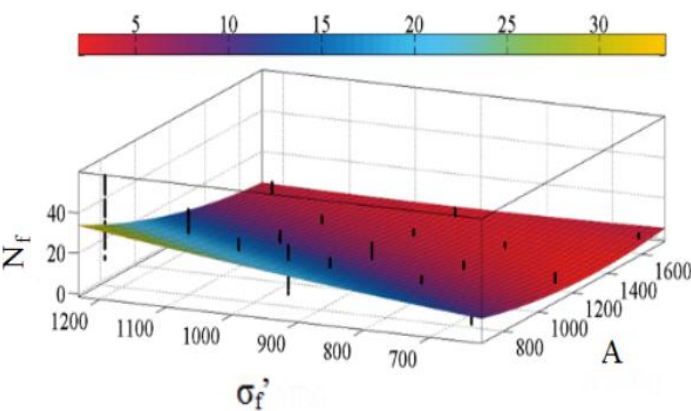

(a) $\mathrm{FF}^{\mathrm{I} / \mathrm{II}}$

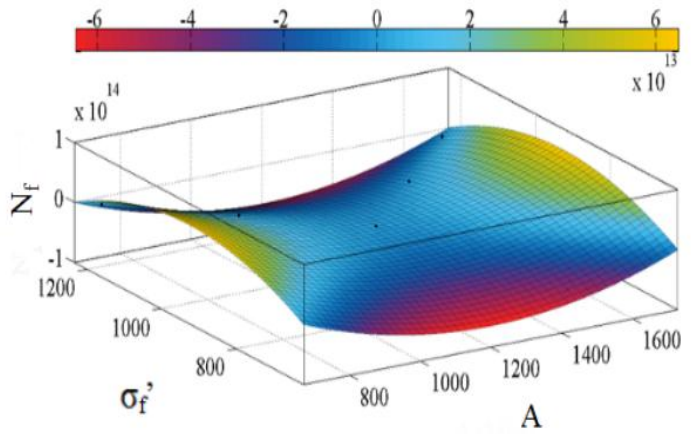

(c) $\mathrm{CCD}^{\mathrm{I} / \mathrm{II}}$

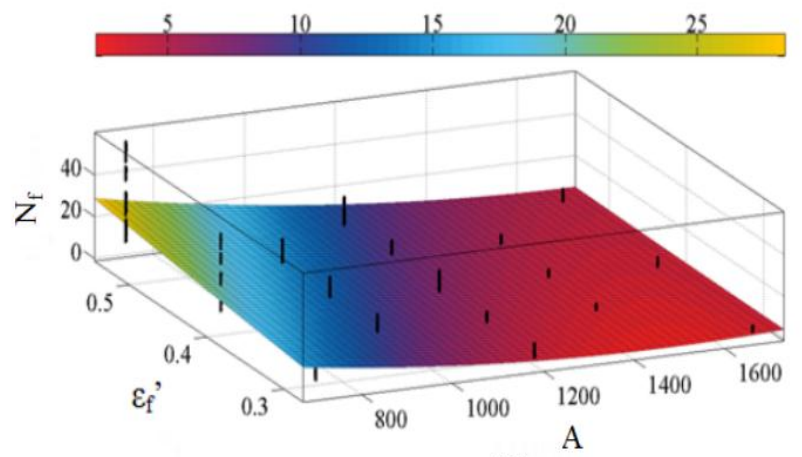

(b) $\mathrm{FF}^{\mathrm{I} / \mathrm{II}}$

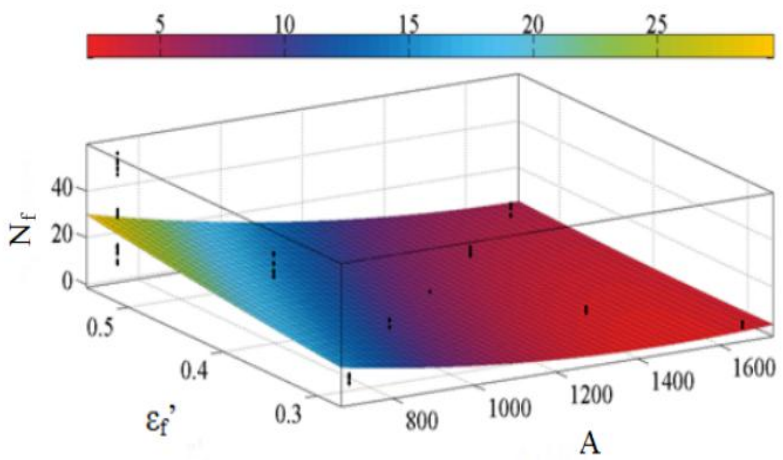

(d) $\mathrm{CCD}^{\mathrm{I} / \mathrm{II}}$

Figura 3.6 - Superfície de Resposta de $P 2$ para (a) e (b) com $\mathrm{FF}^{\mathrm{I} / \mathrm{II}}$ e para (c) e (d) com $\mathrm{CCD}^{\mathrm{I} / \mathrm{II}}$

A Figura 3.6 corresponde a um fatorial $\mathrm{FF}^{\mathrm{I} / \mathrm{II}}$ e $\mathrm{CCD}^{\mathrm{I} / \mathrm{II}}$ a um e dois desvios padrões com comportamento semelhante ao da Figura 3.5. No entanto, devido a um maior número de amostras utilizadas na regressão se torna possível analisar a importância da variável $\varepsilon_{f}$, uma vez que, para esta figura, a mesma tende a apresentar uma curvatura que antes era praticamente inexistente. Além disso, a presença de curvatura é característica de regressão de segunda ordem mostrando a influência da variável no modelo. Já as superfícies de resposta para $L H S$ e $S S$ em $P 2$ são dadas pela Figura 3.7. 


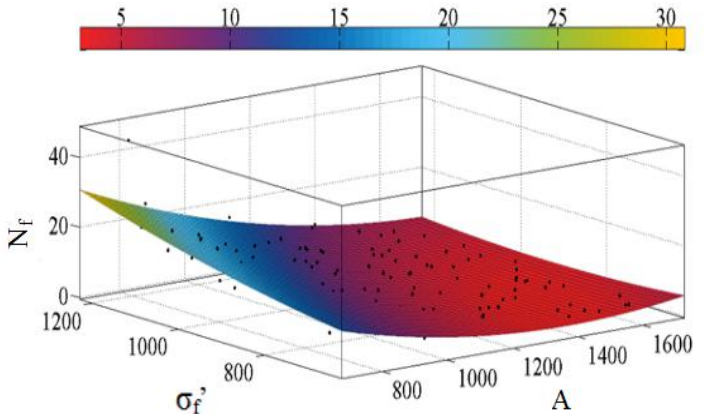

(a) LHS $^{\text {II }}$

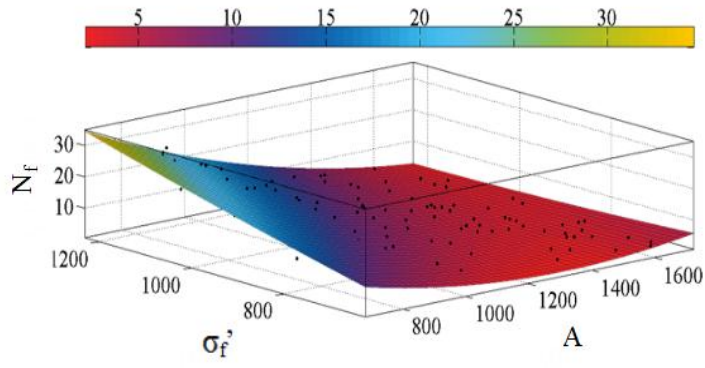

(c) $\mathrm{SS}^{\mathrm{II}}$

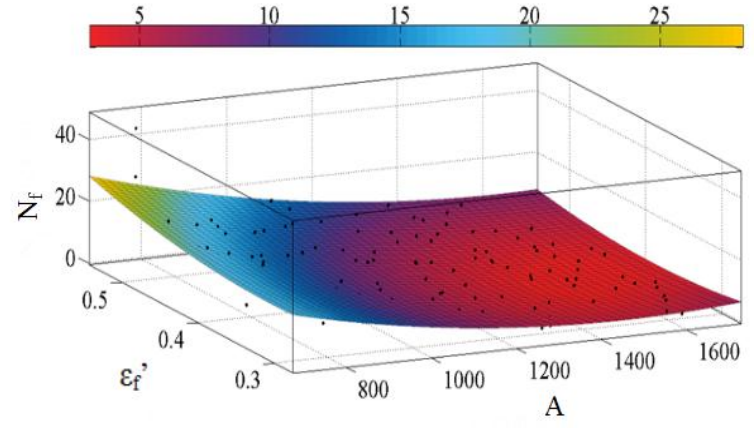

(b) $\mathrm{LHS}^{\mathrm{II}}$

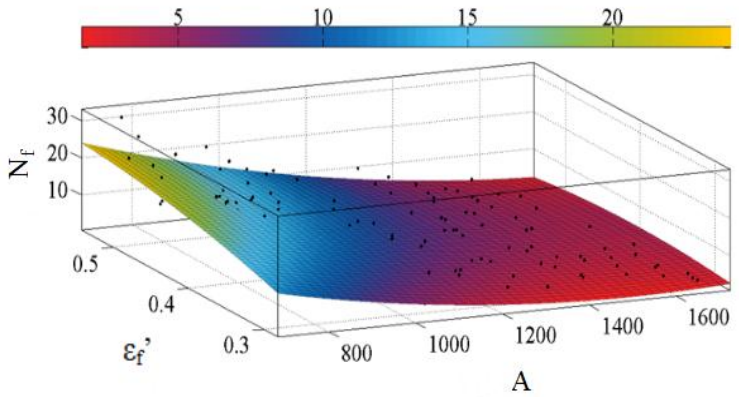

(d) $\mathrm{SS}^{\mathrm{II}}$

Figura 3.7 - Superfície de Resposta de $P 2$ para (a) e (b) LHS ${ }^{\mathrm{II}}$ o e para (c) e (d) com SS ${ }^{\mathrm{II}}$

Na Figura 3.7, as variáveis de entrada utilizadas na determinação das superfícies de resposta são aleatórias a dois desvios padrões para $\operatorname{LHS}^{\mathrm{II}}$ e $\mathrm{SS}^{\mathrm{II}}$. Ainda de acordo com a Figura 3.7, os pontos estão uniformemente distribuídos pela superfície diferenciando-se, portanto, do formato padronizado utilizado pelos experimentos fatoriais. Além disso, a superfície de resposta para ambos os modelos da figura apresentam predominância entre 5 a 10 ciclos de vida à fadiga.

Para a análise do ajuste das $P D F$ s em relação a um Monte Carlo de referência $\left(M C^{R E F}\right)$ considerou-se a equação a seguir, sendo a mesma correspondente ao erro quadrático médio normalizado ou Normalized root mean square error (NRMSE):

$$
N R M S E=1-\frac{\left\|M C^{R E F}-X\right\|}{\left\|M C^{R E F}-\mu\left(M C^{R E F}\right)\right\|}
$$

Na Equação 3.5, observa-se a dependência de $M C^{R E F}$ na validação da distribuição do meta modelo. Além disso, para NRMSE próximos de 1 o ajuste é considerado perfeito e para magnitudes próximas de $-\infty$ passa a ser considerado um ajuste ruim. Na Tabela 3.4 apresentam-se os valores de NRMSE para a Tabela 3.3. 
Tabela 3.4 - Ajuste dos meta modelos de $N_{f}$ para $P 2$

\begin{tabular}{c|c|c|c|c|c|c|c|c|c}
\hline \multirow{2}{*}{ Amostras } & $M C^{R E F}$ & $F F^{I I}$ & $F F^{I / I I}$ & \multirow{2}{*}{$C C D^{I I}$} & $C C D^{I / I I}$ & $L H S^{I I}$ & $S S^{I I}$ & $L H S^{I I I}$ & $S S^{I I I}$ \\
\hline$N$ & - & 6561 & 13121 & 257 & 513 & 125 & 125 & 125 & 125 \\
\hline$N R M S E$ & 1,00 & $-0,49$ & $-0,45$ & $-0,54$ & $-0,49$ & $-0,41$ & $-0,38$ & $-0,46$ & $-0,59$ \\
\hline
\end{tabular}

Nota-se na Tabela 3.4 que os valores de NRMSE encontram-se no mesmo padrão, ou seja, apresentam-se com magnitudes mais próximas de 1 do que à $-\infty$. Outra análise encontra-se na Figura 3.8 para as $P D F s$ de $N_{f}$.

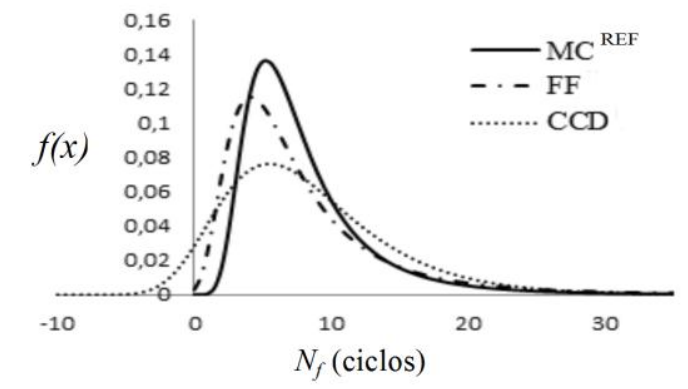

(a) $\mathrm{FF}^{\mathrm{II}}$ e $\mathrm{CCD}^{\mathrm{II}}$

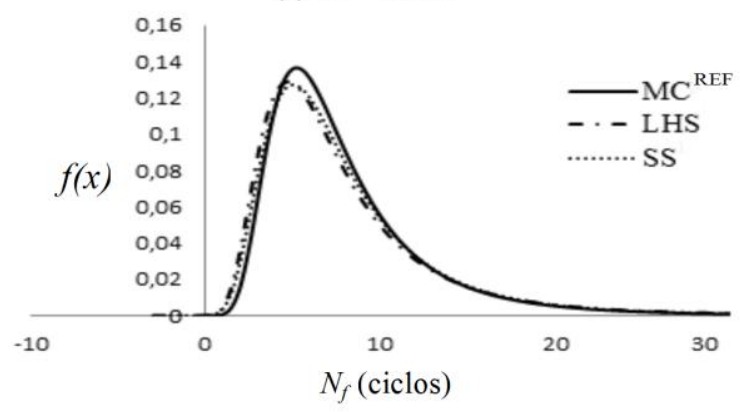

(c) LHS $^{\text {II }}$ e SS ${ }^{\text {II }}$

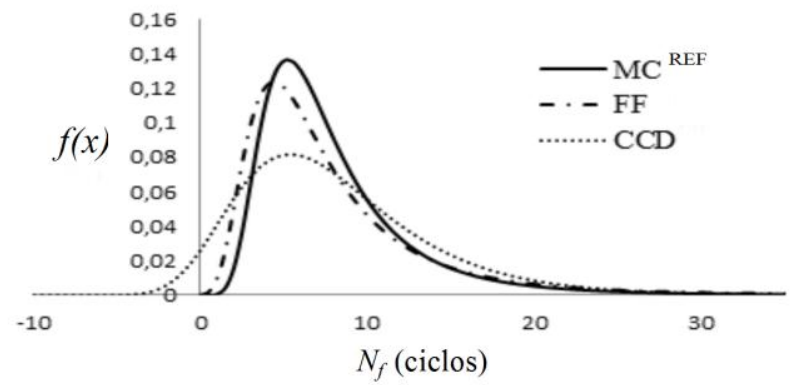

(b) $\mathrm{FF}^{\mathrm{III}}$ e $\mathrm{CCD}^{\mathrm{III}}$

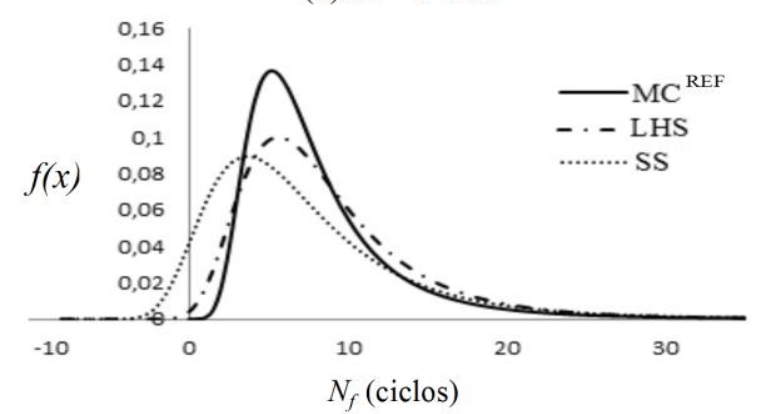

(d) $\mathrm{LHS}^{\mathrm{III}}$ e SS ${ }^{\mathrm{III}}$

Figura 3.8 - PDFs de $N_{f}$ para $P 2$ com (a) $\mathrm{FF}^{\mathrm{II}}$ e $\mathrm{CCD}^{\mathrm{II}}$, (b) $\mathrm{FF}^{\mathrm{I} / \mathrm{II}}$ e $\mathrm{CCD}^{\mathrm{I} / \mathrm{II}}$, (c) $\mathrm{LHS}^{\mathrm{II}}$ e $\mathrm{SS}^{\mathrm{II}}$, (d) LHS $^{\text {III }}$ e SS ${ }^{\text {III }}$.

A regressão das amostras da Tabela 3.3 proporcionou a determinação dos coeficientes dos polinômios para os meta modelos e através destes polinômios pode-se quantificar a incerteza através das funções densidades de probabilidades $(P D F)$. Na Figura 3.8, encontra-se as PDFs dos modelos da Tabela 3.3 e de $L H S$ e $S S$ a três desvios padrões, onde para cada modelo considerou-se 5E+5 simulações de Monte Carlo. A validação das PDFs é realizada através da comparação dos modelos com uma rotina adaptada de Negrão (2014) que também considerou para $5 \mathrm{E}+5$ simulações.

Na verificação de $N_{f}$, os meta modelos se adequaram a uma $G E V$ com um melhor ajuste ao $M C^{R E F}$ de Negrão (2014) nas amostragens de $S S$ e $L H S$ a dois desvios padrões. Além 
disso, percebe-se que $N_{f}<0$ corresponde apenas ao modelo matemático e não pode ser considerado para o modelo físico. Isto acontece, pois o fenômeno de ciclos de vida à fadiga não é quantificado para magnitudes negativas e, portanto, o mesmo, serve apenas para representação do comportamento da distribuição da variável $N_{f}$.

Percebe-se ainda que o uso de valores aleatórios é muito eficiente e que os mesmos apresentam um comportamento muito superior ao experimento fatorial. Além disso, o uso do $L H S$ e $S S$ necessitou um número de amostras inferior ao $F F$ e ao $C C D$ e desta forma, apresentou-se mais eficiente com menor tempo de processamento e com resultados mais precisos.

Uma última análise para $P 2$ consiste em se verificar o desempenho do $L H S$ e do $S S$ para Meta modelos com 2, 3 e 4 desvios padrões na Tabela 3.5. Além disso, o objetivo desta análise é observar o comportamento da distribuição à medida que o domínio das amostras aleatórias aumenta.

Tabela 3.5 - Parâmetros com 2, 3, 4 desvios padrões da regressão de P2

\begin{tabular}{c|c|c|c|c}
\hline $\begin{array}{c}\text { Regressão } \\
N_{f}\end{array}$ & SSE & $\mathrm{r}^{2}$ & RMSE & $\mathrm{N}$ \\
\hline LHS $^{\mathrm{II}}$ & $1,61 \mathrm{E}+2$ & 0,96 & 1,14 & 125 \\
\hline $\mathrm{SS}^{\mathrm{II}}$ & $5,37 \mathrm{E}+1$ & 0,99 & 0,65 & 125 \\
\hline LHS $^{\mathrm{III}}$ & $2,62 \mathrm{E}+2$ & 0,97 & 1,45 & 125 \\
\hline $\mathrm{SS}^{\mathrm{III}}$ & $1,61 \mathrm{E}+3$ & 0,92 & 3,59 & 125 \\
\hline $\mathrm{LHS}^{\mathrm{IV}}$ & $1,31 \mathrm{E}+4$ & 0,87 & 10,25 & 125 \\
\hline $\mathrm{SS}^{\mathrm{IV}}$ & $1,00 \mathrm{E}+5$ & 0,67 & 28,39 & 125 \\
\hline
\end{tabular}

Na Tabela 3.5, encontra-se os parâmetros de regressão polinomial de segunda ordem. Assim, pode-se afirmar que à medida que o domínio diminui, o modelo passa a apresentar um melhor desempenho. Percebe-se também que o domínio entre 4 desvios padrões apresenta-se com uma somatória de erros alta e com coeficiente de determinação inferior a $90 \%$, ou seja, menos de $90 \%$ da variabilidade dos pontos pode ser explicada pela regressão. Além disso, apresenta-se na Tabela 3.6 os parâmetros para $R B F \operatorname{com} 2$ e 3 desvios padrões, sendo $N$ o número de amostras utilizadas para a determinação dos coeficientes e $N C$ o número de coeficientes do meta modelo. 
Tabela 3.6 - Parâmetros com 2 e 3 desvios padrões com $R B F$

\begin{tabular}{c|c|c|c|c|c}
\hline $\begin{array}{c}\text { Regressão } \\
N_{f}\end{array}$ & SSE & $r^{2}$ & $R M S E$ & $N C$ & $N$ \\
\hline LHS $^{\mathrm{II}}$ & $1,72 \mathrm{E}-26$ & 1,0 & $1,17 \mathrm{E}-14$ & 125 & 125 \\
\hline $\mathrm{SS}^{\mathrm{II}}$ & $1,66 \mathrm{E}-25$ & 1,0 & $3,65 \mathrm{E}-14$ & 125 & 125 \\
\hline LHS $^{\mathrm{II}}$ & $1,19 \mathrm{E}-25$ & 1,0 & $3,08 \mathrm{E}-14$ & 125 & 125 \\
\hline $\mathrm{SS}^{\mathrm{II}}$ & $1,69 \mathrm{E}-25$ & 1,0 & $3,68 \mathrm{E}-14$ & 125 & 125 \\
\hline
\end{tabular}

Na Tabela 3.6 observam-se ainda que os parâmetros SSE e RMSE apresentam-se ambos nulos, sendo, portanto, parâmetros que validam o meta modelo. Em contra partida, ao analisar-se o coeficiente de determinação o mesmo não acontece, pois o parâmetro não permite distinção entre os meta modelos. Desta forma, percebe-se que mesmo um bom $r^{2}$, nem sempre permite um meta modelo aceitável. Já a Tabela 3.7 apresenta o processo de interpolação para $R P F P 1$.

Tabela 3.7 - Parâmetros com 2 e 3 desvios padrões com RBFP1

\begin{tabular}{c|c|c|c|c|c}
\hline $\begin{array}{c}\text { Regressão } \\
N_{f}\end{array}$ & $S S E$ & $r^{2}$ & $R M S E$ & $N C$ & $N$ \\
\hline LHS $^{\text {II }}$ & $4,85 \mathrm{E}-25$ & 1,0 & $6,23 \mathrm{E}-14$ & 125 & 125 \\
\hline SS $^{\text {II }}$ & $4,39 \mathrm{E}-25$ & 1,0 & $5,93 \mathrm{E}-14$ & 125 & 125 \\
\hline LHS $^{\text {III }}$ & $1,11 \mathrm{E}-22$ & 1,0 & $9,45 \mathrm{E}-13$ & 125 & 125 \\
\hline SS $^{\text {III }}$ & $1,76 \mathrm{E}-24$ & 1,0 & $1,19 \mathrm{E}-13$ & 125 & 125 \\
\hline
\end{tabular}

Nota-se na Tabela 3.7 que os parâmetros de SSE e RMSE apresentam-se praticamente nulos, ou seja, os meta modelos não apresentam erros residuais. Percebe ainda que para as 125 amostras, o coeficiente de determinação é considerado aceitável, ou seja, 100\% da variabilidade dos pontos podem ser explicados pela interpolação. Porém, em contra partida, observa-se ainda que mesmo $r^{2}=1$ é necessário um parâmetro adicional na validação do modelo. Para as RBFs da Tabela 3.6 apresenta-se a Tabela 3.8.

Tabela 3.8 - Ajuste dos meta modelos de $N_{f}$ para $R B F$

\begin{tabular}{c|c|c|c|c|c}
\hline \multirow{2}{*}{ Amostras } & \multirow{2}{*}{ RC $^{R E F}$} & $\mathcal{L} H S^{I I}$ & \multirow{2}{*}{$S S^{I I}$} & $\mathcal{L} H S^{I I I}$ & $S S^{I I I}$ \\
\hline$N$ & - & 125 & 125 & 125 & 125 \\
\hline NRMSE & 1,00 & $-2,93$ & $-8,23$ & $-1,12$ & $-0,67$ \\
\hline
\end{tabular}

Na Tabela 3.8 percebe-se que o ajuste não é adequado para as $R B F s$, principalmente, para $\mathrm{SS}^{\mathrm{II}}$, pois o mesmo quando comparado a Tabela 3.4 encontra-se muito disperso. Na Tabela 3.9 encontram-se os NRMSE para RBFP1. 
Tabela 3.9 - Ajuste dos meta modelos de $N_{f}$ para $R B F P 1$

\begin{tabular}{|c|c|c|c|c|c|}
\hline Amostras & $M C^{R E F}$ & $L H S^{I I}$ & $S S^{I I}$ & $L H S^{I I I}$ & $S S^{I I I}$ \\
\hline$N$ & - & 125 & 125 & 125 & 125 \\
\hline NRMSE & 1,00 & $-0,39$ & $-0,35$ & $-1,21$ & $-0,80$ \\
\hline
\end{tabular}

Por fim, na Tabela 3.9 nota-se um ajuste melhor devido a adição do polinômio de primeira ordem aos meta modelos com RBFs. Além disso, na Figura 3.9 apresentam-se as PDFs para $R B F P 1$.

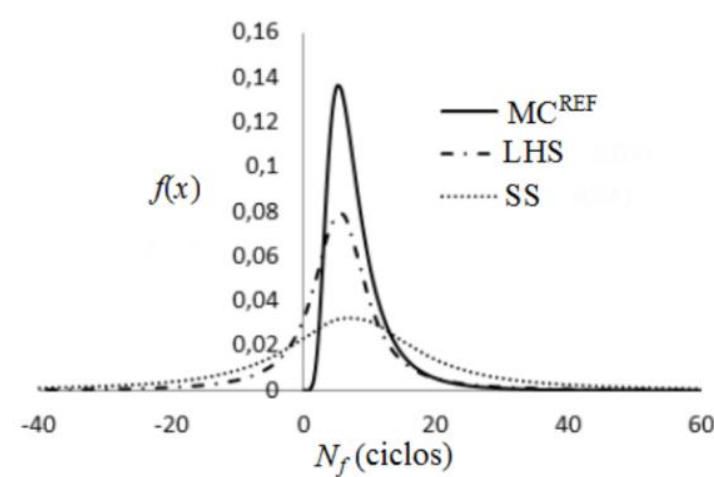

(a) LHS $^{\text {Il }}$ e SS ${ }^{\text {Il }}$

(RBF)

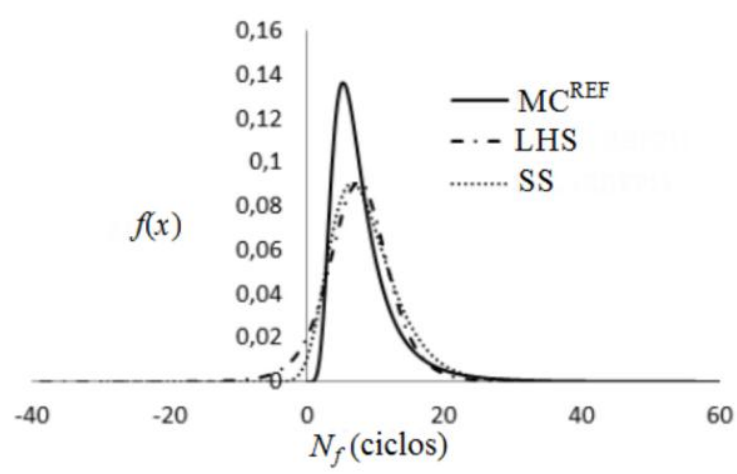

(c) $\mathrm{LHS}^{\text {II }}$ e SS

(RBFP1)

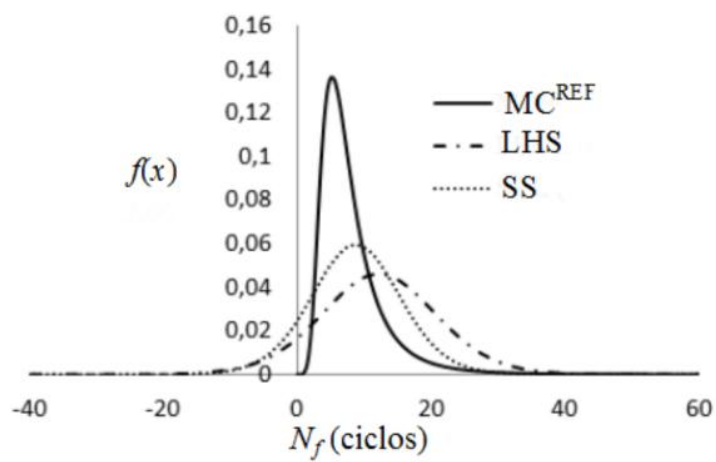

(b) $\mathrm{LHS}^{\mathrm{III}}$ e $\mathrm{SS}^{\text {III }}$

(RBF)

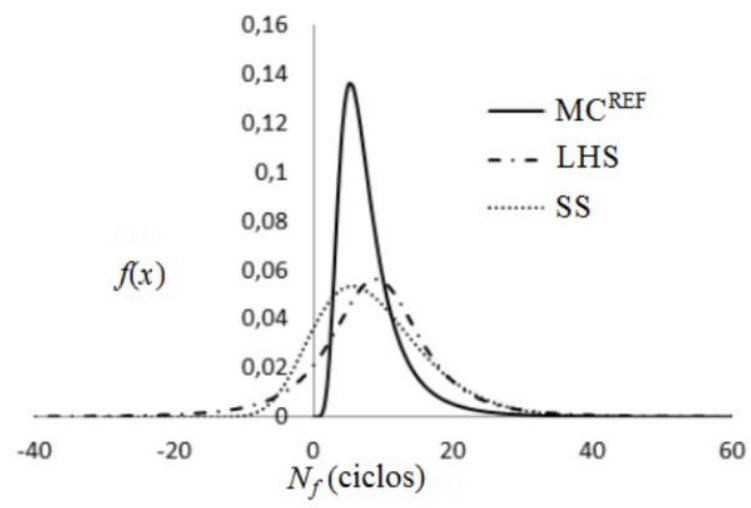

(d) LHS ${ }^{\text {III }}$ e SS ${ }^{\text {II }}$

(RBFP1)

Figura 3.9 - PDFs para (a) e (b) como $R B F$ e para (c) e (d) como $R B F P 1$

Na Figura 3.9 percebe-se que o ajuste das mesmas é melhor quando comparada ao uso de funções polinomiais. Desta forma, ambas as amostragens são adequadas para o processo de interpolação. A validação das $P D F$ s é realizada através da comparação dos modelos com uma rotina adaptada de Negrão (2014) que também considerou para 5E+5 simulações. Observam-se na Figura 3.10 as superfícies de resposta construídas para $R B F$, sendo adotados para as principais variáveis aleatórias da regressão polinomial. 


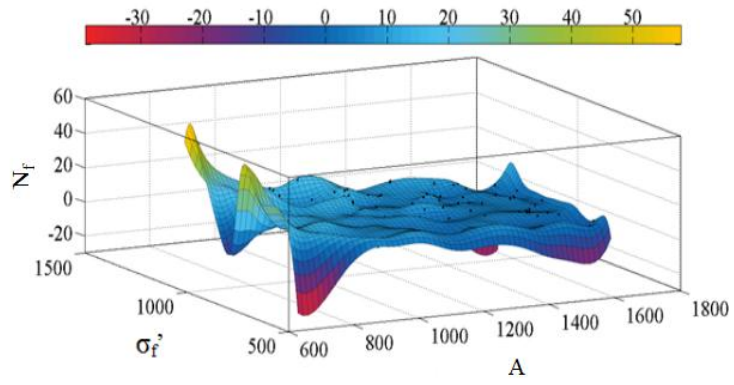

(a) LHS II

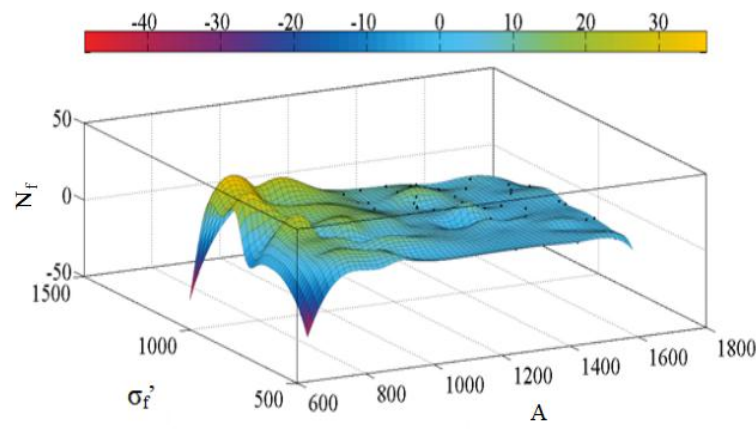

(c) $\mathrm{SS}^{\mathrm{II}}$

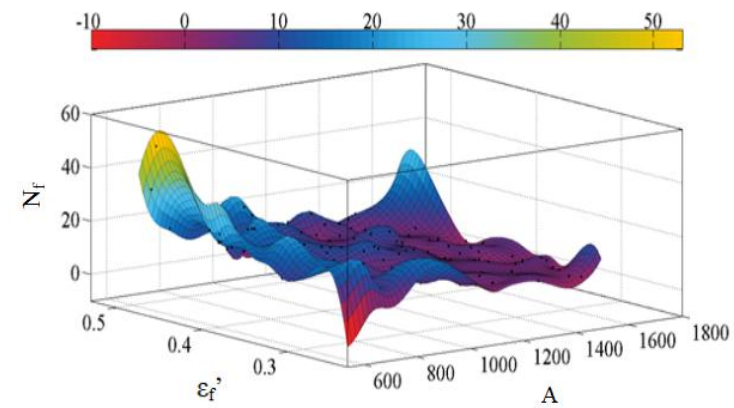

(b) LHS ${ }^{\text {II }}$

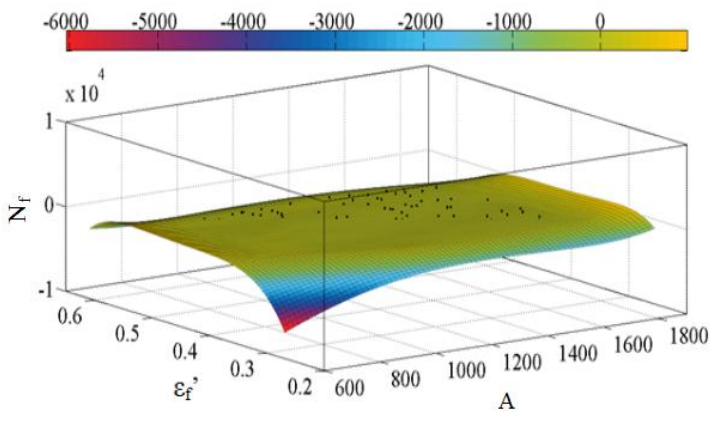

(d) $\mathrm{SS}^{\mathrm{II}}$

Figura 3.10 - Superfície de Resposta para $R B F$ com (a) e (b) $\operatorname{LHS}^{\mathrm{II}}$ e com (c) e (d) $S S^{\mathrm{II}}$.

Nota-se ainda que as superfícies da Figura 3.10 apresentam ondulações, sendo estas ocasionadas devido à interação de cada amostra com o restante do domínio. Por fim, observa-se que para a maioria das superfícies, o número de ciclos varia entre 0 e 10 ciclos de vida. Exibe-se ainda a Figura 3.11 para RBFP1.

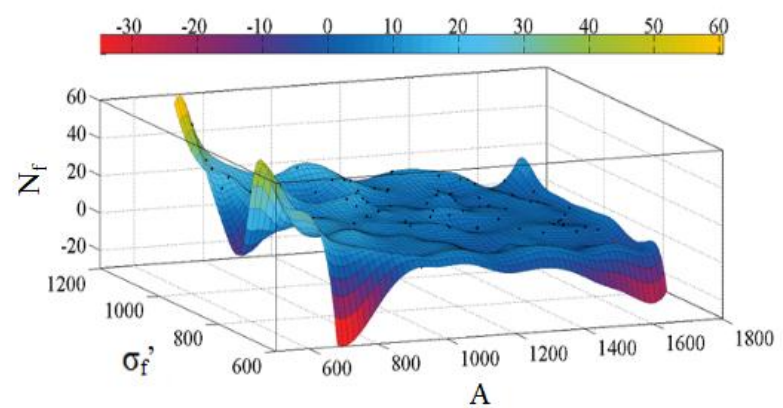

(a) LHS $^{\text {II }}$

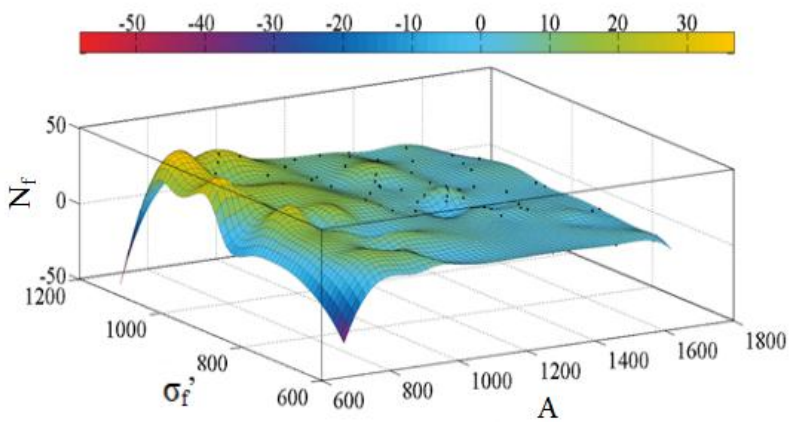

(c) $\mathrm{SS}^{\mathrm{II}}$

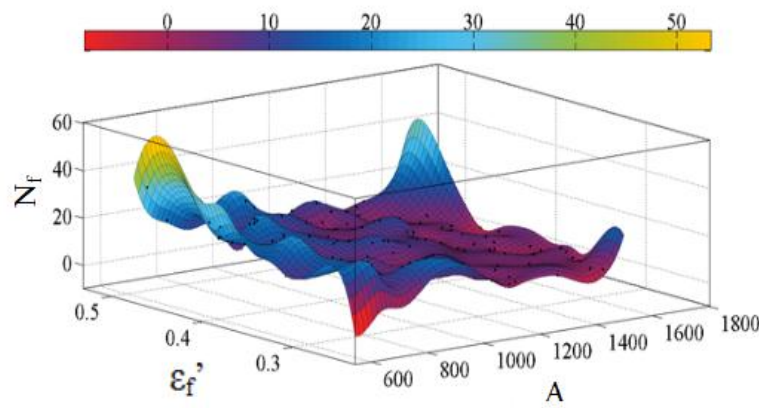

(b) LHS ${ }^{\text {II }}$

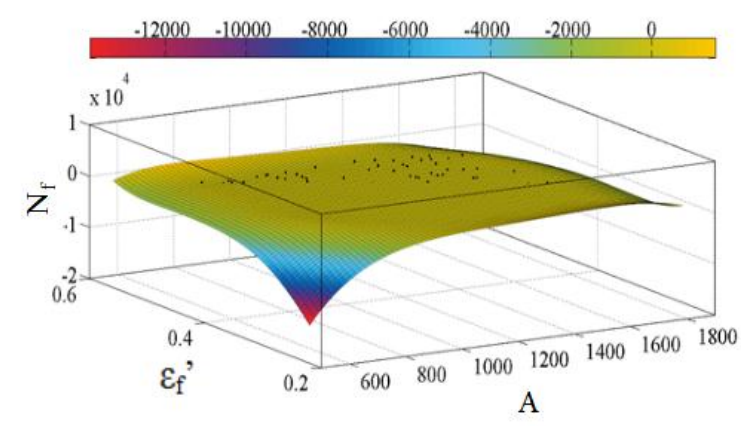

(d) $\mathrm{SS}^{\text {II }}$

Figura 3.11 - Superfície de Resposta para RBFP1 para (a) e (b) LHS e para (c) e (d) SS 
Na Figura 3.11 encontram-se as superfícies de resposta construídas para $R B F P 1$, sendo adotados para as principais variáveis aleatórias da regressão polinomial. Observam-se que para a maioria das superfícies, o número de ciclos varia entre 0 e 10 ciclos de vida, sendo semelhante ao de $R B F$. 


\section{QUANTIFICAÇÃO DA INCERTEZA DA TAXA DE LIBERAÇÃO DE ENERGIA DEPENDENTE DO TEMPO DE UM MEMBRO ESTRUTURAL COM TRINCA}

\subsection{INTRODUÇÃO}

Nesta seção apresentam-se trabalhos publicados com meta modelos para análise de fenômenos da mecânica da fratura na literatura atual. Recentemente, diversos trabalhos foram publicados; destacando-se Bayramov et al. (2004), Gu et al. (2011), Leonel et al. (2011), Wang et al. (2010), Yuvaraj et al. (2013), Badalló et al. (2015), Dey et al. (2016) e Tan e Hsu (2016) . Além disso, é importante salientar que os trabalhos publicados apresentam como meta modelos superfícies de resposta polinomiais e interpolações por funções de base radial.

$\mathrm{Na}$ análise de meta modelos com superfície de resposta polinomial destacam-se os de Leonel et al. (2011) e Dey et al. (2016). No caso de Leonel et al. (2011) a metodologia consiste no uso de confiabilidade em estruturas, focando-se no uso de superfície de respostas para solução de problemas de propagação aleatória de trinca de forma a analisar a eficiência dos meta modelos. Outra pesquisa de grande interesse é a Dey et al. (2016), o qual descreve uma estratégia sistemática para identificação da posição de trincas e do centro de gravidade em vigas em perfil metálico em balanço através da modelagem em elementos finitos para construção da superfície de resposta. Além disso, outro tipo de metodologia bastante usado consiste nas funções de base radial, sendo a de Wang et al. (2010), Gu et al. (2011), Badalló et al. (2015) e Tan e Hsu (2016) algumas publicações recentes.

O uso de funções de base radial é bem comum em meta modelos para análise da mecânica da fratura destacando-se publicações de Gu et al. (2011) e Badalló et al. (2015). No caso de Gu et al. (2011) é desenvolvido RBFs trigonométricas para análise do campo de tensões na ponta da trinca. Já para Badalló et al. (2015), o uso de meta modelos é realizado para otimização de danos à tração com um estudo preliminar das variáveis de entrada; tendo como objetivo analisar a influência das mesmas em aberturas de trincas. Além disso, o corpo de prova em estudo é modelado em elementos finitos para que assim servisse como amostragem para interpolação em funções de base radial. 
Para este trabalho o uso de meta modelos é realizado através de amostragens aleatórias. Além disso, percebe-se que ainda há uma necessidade do estudo do método em fratura e, portanto, o presente trabalho torna-se de grande importância.

\subsection{ESTUDO DE CASO DE PLACA 3D}

\subsubsection{Parâmetros do modelo}

O modelo a ser considerado consiste em uma placa conforme a Figura 4.1, onde se apresenta a geometria da mesma; sendo $\beta$ a inclinação da trinca, $W$ o comprimento da placa, $a$ espessura da trinca, $2 c$ o comprimento da trinca, $e$ a espessura da placa, $\phi$ o ângulo que informa a posição na frente da superfície da trinca, $x$ e $y$ os eixos do plano inicial e $x$ ' e $y^{\prime}$ os eixos do plano principal da trinca.

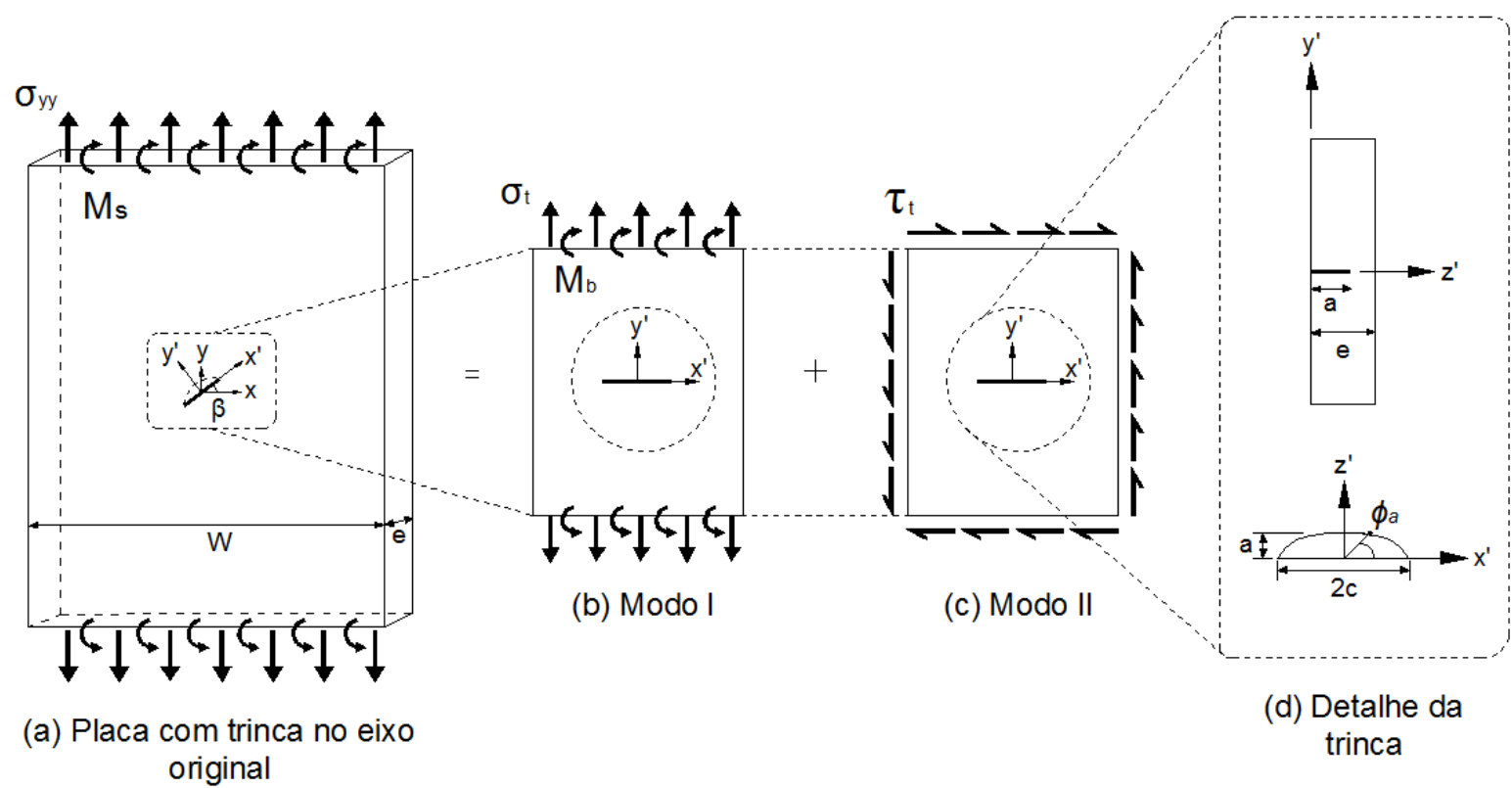

Figura 4.1 - Geometria para (a) placa com trinca no eixo original, (b) Modo I, (c) Modo II e (d) Detalhe da trinca

A Figura 4.1 ainda consiste em uma representação do Modo misto que surge, como mencionado anteriormente devido à posição das tensões atuantes. A placa é solicitada a uma tensão axial $\sigma_{y y}$ e a um momento $M_{s}$ dada na Figura 4.1a, sendo o mesmo responsável por induzir a uma tensão $\sigma_{s}$. Já na Figura 4.1 b percebe-se apenas o Modo I solicitado por uma tensão $\sigma_{t}$ e por um momento $M_{b}$. No caso do $M_{b}$, o mesmo resulta na tensão de flexão $\sigma_{b}$ de acordo com a Equação (4.1). Na Figura 4.1c encontra-se o Modo II ocasionado por uma tensão $\tau_{t}$ e na Figura 4.1d o detalhe da trinca. Porém, neste trabalho a análise 
estrutural será realizada apenas para Modo I, pois o mesmo influencia mais neste tipo de análise.

$$
\sigma_{b}=\frac{6 M_{b}}{W e^{2}}
$$

em que:

$$
\begin{aligned}
& M_{b}=M_{s} \cos ^{2} \beta \\
& \sigma_{t}=\sigma_{y y} \cos ^{2} \beta
\end{aligned}
$$

As Equações (4.1) a (4.2a-b) representam para o Modo I as tensões atuantes para uma inclinação de trinca $\beta$. Para quantificar o fator de intensidade de tensão $\left(K_{I}\right)$, Newman e Raju (1981) elaboraram uma formulação empírica resultada de modelagem computacional no método dos elementos finitos, sendo a mesma representada pela Equação (4.3).

$$
K_{I}=\left(\sigma_{t}+\sigma_{b} H_{p}\right) \sqrt{\frac{\pi a}{Q}} F\left(\frac{a}{e}, \frac{a}{c}, \frac{c}{W}, \phi_{a}\right)=\sigma_{w} D_{o}
$$

Com:

$$
\begin{array}{ll}
Q=1+1.464\left(\frac{a}{c}\right)^{1.65} & \text { para } \frac{a}{c} \leq 1 \\
Q=1+1.464\left(\frac{c}{a}\right)^{1.65} & \text { para } \frac{c}{a}>1
\end{array}
$$

$\mathrm{e}$

$$
\begin{aligned}
& \sigma_{w}=\left(\sigma_{t}+\sigma_{b} H_{p}\right) \\
& D_{o}=\sqrt{\frac{\pi a}{Q} F\left(\frac{a}{e}, \frac{a}{c}, \frac{c}{W}, \phi_{a}\right)}
\end{aligned}
$$

Segundo a Equação (4.3), $Q$ corresponde a um parâmetro que pode ser resolvido pela Equação (4.4a-b), e $F$ corresponde a uma a função de correção que depende das relações $a / e, a / c, c / W$ e da inclinação angular $\phi_{a}$ que descreve a posição de análise da trinca em função do comprimento e profundidade da mesma. Desta forma, a função $F$ pode ser facilmente analisada através da Equação (4.6). 


$$
F=\left[M_{1}+M_{2}\left(\frac{a}{e}\right)^{2}+M_{3}\left(\frac{a}{e}\right)^{4}\right] f_{\phi_{a}} g f_{w}
$$

Com:

$$
\begin{aligned}
& M_{1}=1,13-0,09\left(\frac{a}{c}\right) \\
& M_{2}=-0,54+\frac{0,89}{0,2+(a / c)} \\
& M_{3}=0,5-\frac{1,0}{0,65+(a / c)}+14\left(1,0-\frac{a}{c}\right)^{24} \\
& f_{\phi_{a}}=\left[\left(\frac{a}{c}\right)^{2} \cos ^{2} \phi_{a}+\sin ^{2} \phi_{a}\right]^{1 / 4} \\
& g=1+\left[0,1+0,35\left(\frac{a}{e}\right)^{2}\right]\left(1-\sin \phi_{a}\right)^{2} \\
& f_{w}=\left[\sec \left(\frac{\pi \cdot c}{W}\right) \sqrt{\frac{a}{e}}\right]
\end{aligned}
$$

As Equações (4.7a-f) descrevem de maneira explicita o comportamento da função $F$. Percebe-se ainda que a mesma depende de funções $M_{1}, M_{2}$ e $M_{3}$, sendo cada uma depende da relação $a / c$. Além disso, a função $F$ depende das funções $f_{\phi a}, f_{w}$ e $g$, que estão relacionadas a $\phi_{a}, a / c$ e $a / e$.

$$
H_{p}=H_{1}+\left(H_{2}-H_{1}\right) \sin ^{p} \phi_{a}
$$

Com:

$$
\begin{aligned}
& p=0,2+\left(\frac{a}{c}\right)+0,6\left(\frac{a}{e}\right) \\
& H_{1}=1-0,34\left(\frac{a}{e}\right)-0,11\left(\frac{a}{c}\right)\left(\frac{a}{e}\right)
\end{aligned}
$$




$$
\begin{aligned}
& H_{2}=1+G_{1}\left(\frac{a}{e}\right)+G_{2}\left(\frac{a}{e}\right)^{2} \\
& G_{1}=-1,22-0,12\left(\frac{a}{c}\right) \\
& G_{2}=0,55-1,05\left(\frac{a}{c}\right)^{3 / 4}+0,47\left(\frac{a}{c}\right)^{3 / 2}
\end{aligned}
$$

Um último conjunto de equações é dado pelas Equações (4.8) e (4.9a-e), sendo as mesmas utilizadas para o parâmetro de flexão $H_{p}$. Nas Equações (4.9a-e), é verificado o parâmetro $p$ sendo que o mesmo depende das relações $a / c$ e a/e e os parâmetros $H_{1}$ e $H_{2}$ que se assemelham a $p$. Além disso, o fenômeno a ser analisado consiste na taxa de liberação de energia $\left(G_{I}\right)$ que corresponde à energia necessária para a propagação de uma trinca. Para $G_{I}$, verifica-se a Equação (4.10).

$$
G_{I}=\frac{K_{I}^{2}}{\bar{E}_{m}}
$$

Em que $\bar{E}_{m}$ corresponde ao modulo de elasticidade e $K_{I}$ ao fator de intensidade de tensão no Modo I. O modulo de elasticidade é dado por:

$$
\overline{\mathrm{E}}_{\mathrm{m}}=\left\{\begin{array}{lr}
\mathrm{E}_{\mathrm{m}} & \text { (Estado Plano de Tensão) } \\
\frac{\mathrm{E}_{\mathrm{m}}}{1-v^{2}} & (\text { Estado Plano de Deformação) }
\end{array}\right.
$$

sendo $v$ correspondente ao coeficiente de Poisson. A Equação (4.11a-b), é adotada para o Estado Plano de Tensões e o Estado Plano de Deformações respectivamente.

\section{Funcão Fluência do concreto a compressão}

Fluência é a dependência do tempo das deformações do material, ocorrendo sobre uma aplicação prolongada do carregamento. Na fluência mesmo sobre carregamentos constantes no tempo, as deformações continuam aumentando. Um modelo simplificado de Bažant e Panula (1978 apud Atrushi, 2003) é utilizado para descrever a função fluência ou creep compliance function $J\left(t, t_{c}\right)$. 


$$
J\left(t, t_{c}\right)=J_{0}\left[1+\varphi_{o}\left(t_{c}\right)^{-d}(\theta)^{p_{o}}\right]
$$

Com:

$$
\theta=t-t_{c}
$$

Nas Equações (4.12) e (4.13), $J\left(t, t_{c}\right)$ depende do tempo de aplicação do carregamento $t_{c} \mathrm{e}$ da idade do concreto $t$. A componente instantânea, $J_{o}$, pode ser definida por:

$$
J_{0}\left(t_{c}\right)=W_{1} e^{-0.4166 t_{c}}+W_{2} e^{-0.01358_{c}}
$$

A componente instantânea $J_{0}\left(t_{c}\right)$ surge de um processo das deformações instantâneas dos dados experimentais de Atrushi (2003) para os tempos de carregamento de 1, 3 e 8 dias da mistura BASE-5 na Figura 4.2.

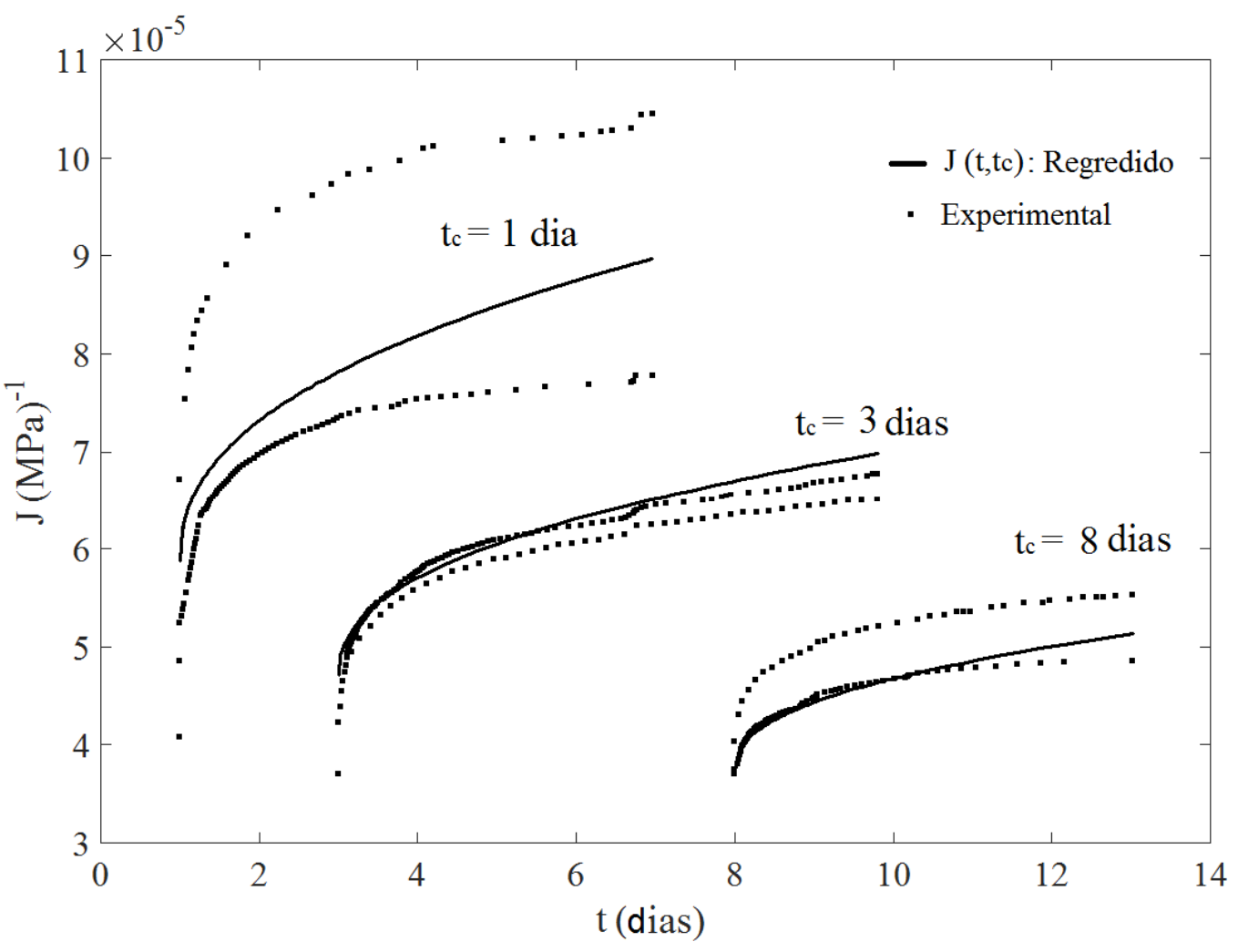

Figura 4.2 - Função fluência regredida, $J\left(t, t_{c}\right)$, e dados experimentais para diferentes $t_{c}$

$\mathrm{Na}$ Figura 4.2, observa-se que cada $t_{c}$ relaciona-se a duas amostras. Percebe-se ainda a grande variabilidade nos resultados experimentais para $t_{c}=1$ dia, como esperado para este tempo prematuro de carregamento. Além disso, a Equação (4.14) representa a regressão da Figura 4.3, sendo $W_{1}$ e $W_{2}$ parâmetros. 


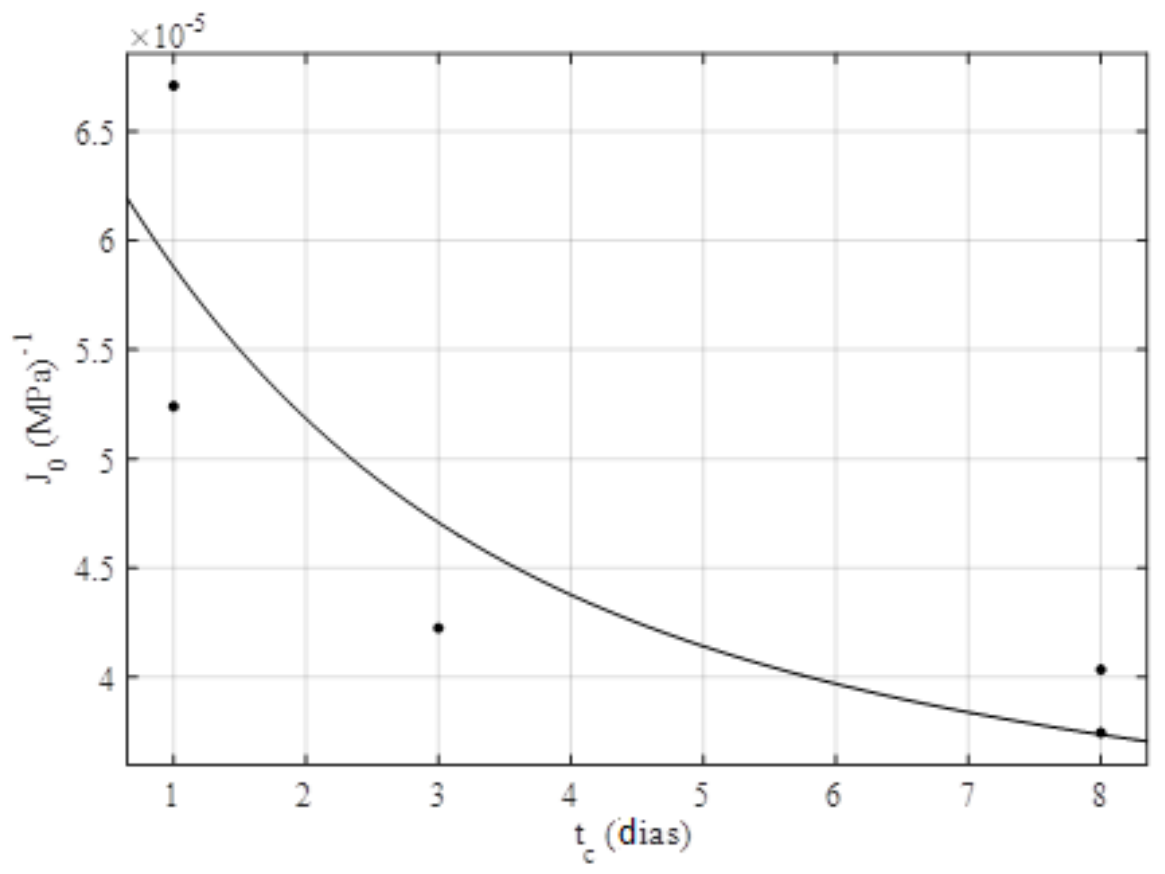

Figura 4.3 - Componente Instantânea, $J_{0}\left(t_{c}\right)$

Na Figura 4.3 nota-se que para $t_{c}=3$ dias, a componente instantânea é a mesma para ambas as amostras. Na Equação (4.12), os parâmetros $p_{o}, d$ e $\varphi_{o}$ surgem da regressão das amostras experimentais, sendo a superfície de resposta de $J\left(t, t_{c}\right)$ apresentada na Figura 4.4 para tempos $t_{c}$ diferentes do inicial.

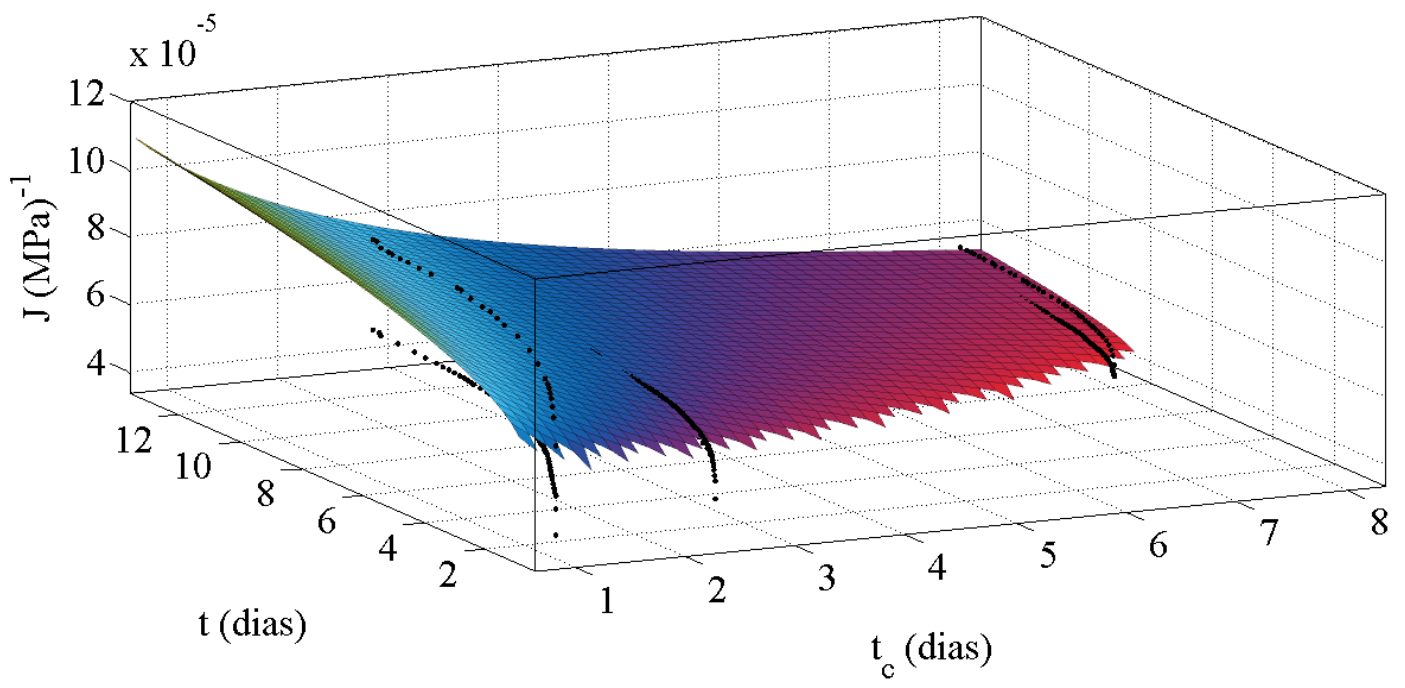

Figura 4.4 - Função fluência, $J\left(t, t_{c}\right)$, para os dias $t_{c}$ de carregamento

Para quantificar $G_{I}$ em meios viscoeláticos considera-se $K_{I}$ e $\bar{E}_{m}$ dependentes do tempo, sendo demostrado desenvolvendo-se as Equações (4.3) e (4.10). 


$$
G_{I}\left(t, t_{c}\right)=\frac{K_{I}^{2}(t)}{\bar{E}_{m}\left(t, t_{c}\right)}=D_{0}^{2} \int_{0}^{t} J(t-\tau) \frac{d}{d \tau}\left(\sigma_{w}^{2}\right) d \tau
$$

\subsubsection{Construção dos meta modelos}

Nesta subseção discute-se a metodologia para análise da construção dos meta modelos para $G_{I}\left(t, t_{c}\right)$. Na Figura 4.5 apresenta-se um fluxograma com a respectiva metodologia para quantificação da incerteza.

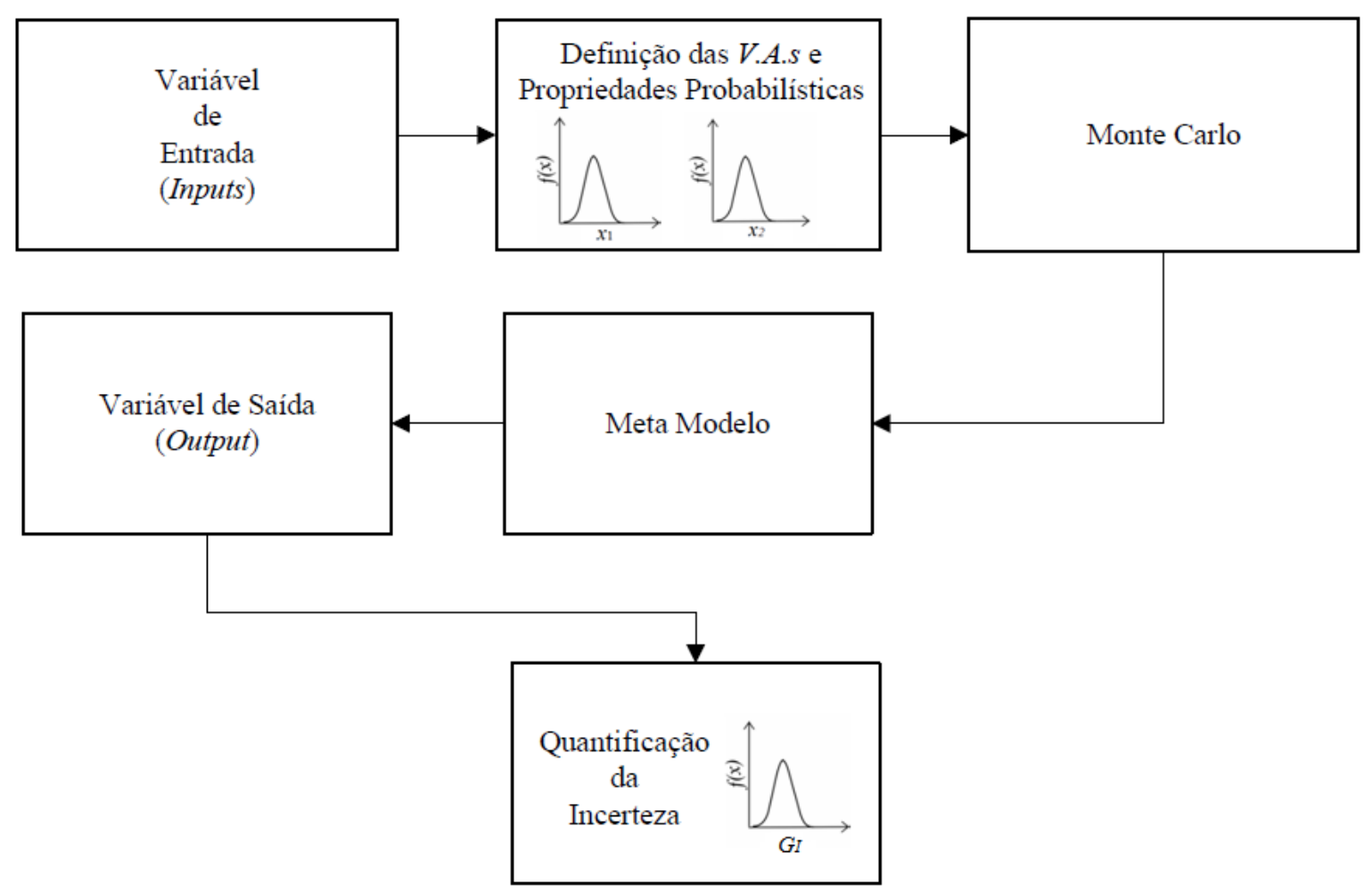

Figura 4.5 - Fluxograma para quantificação da incerteza de $G_{I}$

Para construção dos coeficientes dos meta modelos considerou-se o mesmo processo da Figura 3.2. Porém, na análise da incerteza dado na Figura 4.5, nota-se a necessidade de novos dados de entrada para o modelo físico de $G_{I}$. Assim, pode-se então realizar a simulação de Monte Carlo e posteriormente quantificar a incerteza. Os parâmetros do modelo são dados pelas variáveis determinísticas da Tabela 4.1:

Tabela 4.1 - Variáveis determinísticas

\begin{tabular}{c|c|c}
\hline Var & Unid & Valor \\
\hline$W$ & $m m$ & 500 \\
\hline$e$ & $m m$ & 100 \\
\hline$\phi_{a}$ & Graus & $90^{\circ}$ \\
\hline
\end{tabular}


As variáveis aleatórias correspondem a parcela das amostragens e são apresentadas na Tabela 4.2. Na Tabela 4.2, nota-se 11 variáveis aleatórias do tipo geométrico, de carregamento e de propriedade do material. Além disso, a tabela contém as respectivas média $(\mu)$, desvio padrão $(\sigma)$, coeficiente de variação $(\mathrm{CV})$ e o tipo de função densidade de probabilidade que será adotada.

Tabela 4.2 - Variáveis aleatórias

\begin{tabular}{c|c|c|c|c|c|c}
\hline Parâmetro & Variável & Unid & $\mu$ & $\sigma$ & $C V$ & $f(x)$ \\
\hline \multirow{4}{*}{ Geométrico } & $a / c$ & - & 0,4 & 0,08 & $20 \%$ & GEV I (máx) \\
\cline { 2 - 7 } & $a / e$ & - & 0,20 & 0,04 & $20 \%$ & GEV I (máx) \\
\cline { 2 - 7 } & $c / W$ & - & 0,10 & 0,02 & $20 \%$ & Normal \\
\cline { 2 - 7 } & $\beta$ & Graus & $45^{\circ}$ & $6,75^{\circ}$ & $15 \%$ & Normal \\
\hline \multirow{3}{*}{ Carregamento } & $\sigma_{y y}$ & $M P a$ & 4,0 & 0,8 & $20 \%$ & GEV I (máx) \\
\cline { 2 - 7 } & $\sigma_{s}$ & $M P a$ & 0,80 & 0,16 & $20 \%$ & Normal \\
\hline \multirow{4}{*}{ Material } & $W_{1}$ & - & $2,853 \mathrm{E}-5$ & $3,685 \mathrm{e}-05$ & $129,16 \%$ & Normal \\
\cline { 2 - 7 } & $W_{2}$ & - & $4,052 \mathrm{E}-5$ & $1,781 \mathrm{e}-05$ & $43,95 \%$ & Normal \\
& $\varphi_{o}$ & - & 0,2448 & 0,0197 & $8,05 \%$ & Normal \\
\cline { 2 - 7 } & $d$ & - & 0,1306 & 0,0625 & $47,86 \%$ & Normal \\
\cline { 2 - 7 } & $p_{o}$ & - & 0,4293 & 0,0522 & $12,18 \%$ & Normal \\
\hline
\end{tabular}

$\mathrm{Na}$ Tabela 4.2, nota-se que as variáveis aleatórias apresentam-se com distribuições do tipo Normal e GEV I. Além disso, os parâmetros do material surgem de uma regressão de $J\left(t, t_{c}\right)$. Os parâmetros de carregamento e de geometria foram determinados para este trabalho através de exemplos da literatura. Observa-se ainda na Tabela 4.2 uma grande variabilidade dos parâmetros do material. Isto ocorre devido ao concreto ser de pouca idade e suas componentes de ajuste de $J\left(t, t_{c}\right)$ apresentarem grande variabilidade no primeiro dia de carregamento. Os respectivos $\theta$ apresentam-se para 1, 7 e 14 dias na Figura 4.6, sendo estes para $(\mu)$ das variáveis aleatórias na determinação de $G_{I}\left(t, t_{c}\right)$.

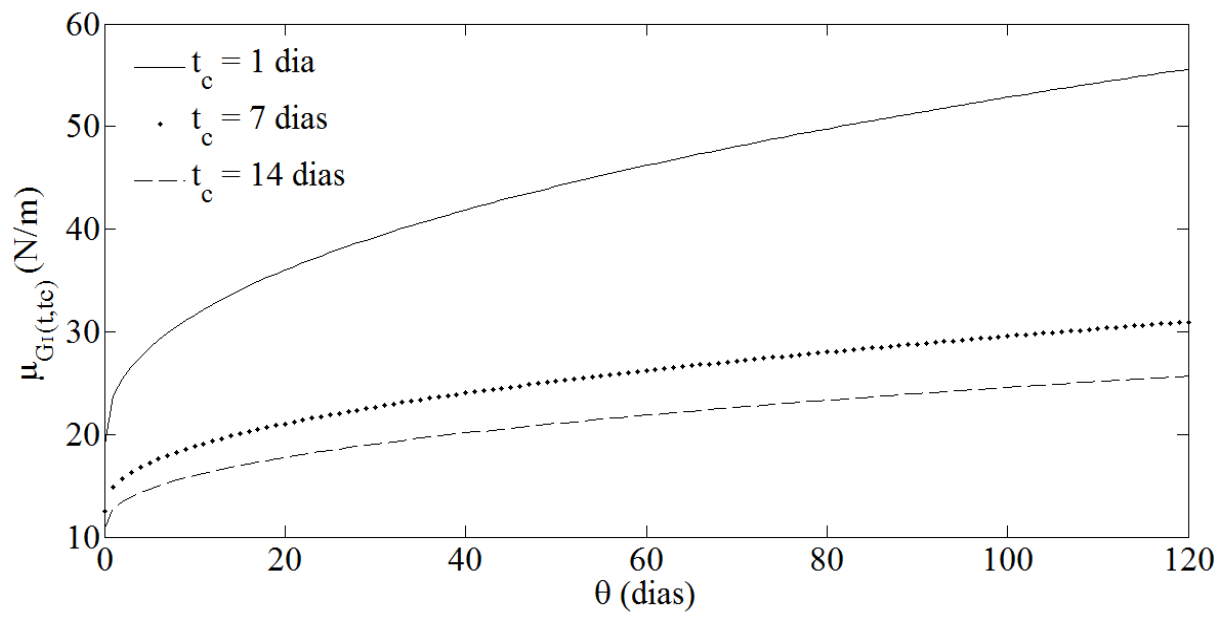

Figura 4.6 Média da taxa de liberação de energia, $\mu_{\mathrm{GI}(\mathrm{t}, \mathrm{tc})}$, utilizando $\mu$ das variáveis aleatórias 
Observa-se na Figura 4.6 que a magnitude de $\mu_{G l(t, t c)}$ tende a aumentar com o aumento do tempo reduzido $(\theta)$. Nota-se ainda que os valores menores de $t_{c}$ apresentam maiores magnitudes de $\mu_{G l}(t, t c)$ ocasionado pelas propriedades de $J\left(t, t_{c}\right)$, pois em tempos inicias, a amostra apresenta uma maior variabilidade das propriedades do material. No caso das estratégias de amostragens das variáveis aleatórias de $L H S$ e $S S$ considera-se a Tabela 4.3 em função de $\sigma$.

\begin{tabular}{|c|c|c|}
\hline Cenário & $\sigma$ & $\begin{array}{c}\% \\
\text { Amostras }\end{array}$ \\
\hline \multirow{2}{*}{$C_{I}$} & {$[-3,3]$} & 70 \\
\hline & {$[-5,5]$} & 30 \\
\hline \multirow{2}{*}{$C_{I I}$} & {$[-3,3]$} & 80 \\
\hline & {$[-5,5]$} & 20 \\
\hline \multirow{4}{*}{$C_{I I I}$} & {$[-1,1]$} & 70 \\
\hline & {$[-2,-1]$ e $[1,2]$} & 20 \\
\hline & {$[-3,-2]$ e $[2,3]$} & 5 \\
\hline & {$[-5,-3]$ e $[3,5]$} & 5 \\
\hline
\end{tabular}

Na Figura 4.7 e na Tabela 4.3, nota-se que o primeiro cenário $\left(\mathrm{C}_{\mathrm{I}}\right)$ corresponde a uma combinação no espaço codificado. Já $\mathrm{C}_{\mathrm{II}}$ é semelhante ao $\mathrm{C}_{\mathrm{I}}$, diferenciando apenas na porcentagem amostral. Por fim, nota-se o cenário $C_{I I I}$ que corresponde a uma estratificação de pontos de [-5,5], onde uma maior quantidade amostral encontra-se no domínio de [-1,1].

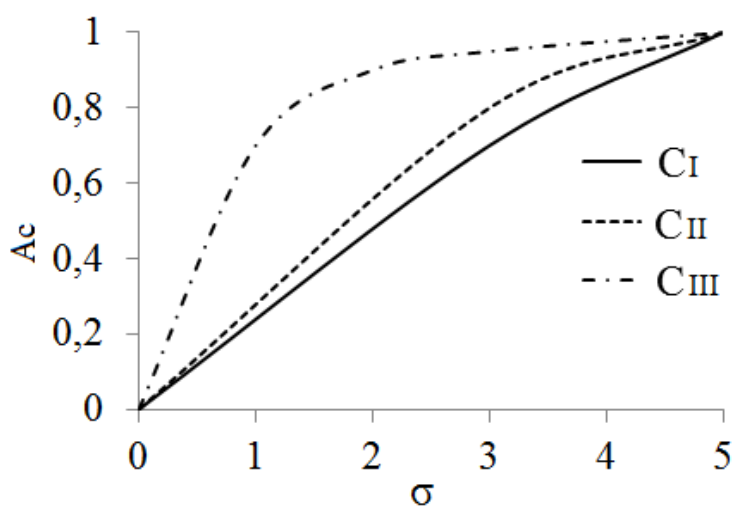

Figura 4.7 - Cenários de amostragem

Na Figura 4.7 notam-se os cenários de amostragens acumulados $\left(A_{c}\right)$ para cada cenário considerado neste trabalho. Cada $A_{c}$ apresenta magnitude máxima de 1 , ou seja, corresponde a $100 \%$ dos pontos da amostras. 


\subsection{RESULTADOS}

Nesta seção, apresentam-se os resultados da predição de $G_{I}$ para diferentes idades de carregamento, sendo adotados LHS e SS em $C_{I}, C_{I I}$ e $C_{I I I}$. Além disso, consideraram-se três casos diferentes para $\theta=10$ dias. Uma primeira análise consiste para $t_{c}=1$ dia $\mathrm{e} t=$ 11 dias. Em seguida, utiliza-se $t_{c}=7$ dias e $t=17$ dias. Por fim, o último caso consiste na verificação para $t_{c}=14$ dias e $t=24$ dias. Outro parâmetro importante consiste no suporte $(\delta)$, sendo adotado $\delta=1$ para as RBFs e RBFPl do tipo Wendland-C2. Na Figura 4.8 notam-se as PDFs para o Monte Carlo de referência $\left(\mathrm{MC}^{\mathrm{REF}}\right)$ que fora construído por meio da Equação (4.15).

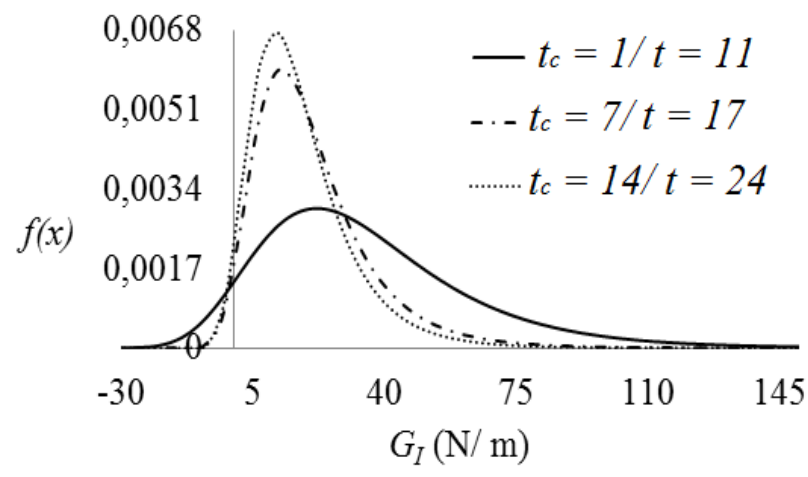

Figura 4.8 - PDFs de $M C^{R E F}$ para $G_{I}$

Na Figura 4.8 nota-se a diminuição dos parâmetros $\mu$ e $\sigma$ à medida que o concreto apresenta maiores idades de carregamento. Como esperado, o processo de solidificação do material influencia na sua rigidez e consequentemente em suas propriedades mecânicas. Além disso, percebe-se que $G_{I}<0$ corresponde apenas ao modelo matemático e não pode ser considerado para o modelo físico. Isto acontece, pois o fenômeno de taxa de liberação de energia não é quantificado para magnitudes negativas e, portanto, o mesmo, serve apenas para representação do comportamento da distribuição da variável $G_{I}$. Já na Tabela 4.4 apresentam-se os parâmetros de $\mu$ e $\sigma$ de $M C^{R E F}$ da Figura 4.8.

Tabela 4.4 - Parâmetros de $\mu$ e $\sigma$ de $M C^{R E F}$

\begin{tabular}{ccc}
\hline Casos & $\mu$ & $\sigma$ \\
\hline$t_{c}=1 / t=11$ & 34,97 & 32,48 \\
\hline$t_{c}=7 / t=17$ & 20,90 & 18,01 \\
\hline$t_{c}=14 / t=24$ & 17,77 & 15,56 \\
\hline
\end{tabular}

Nota-se na Tabela 4.4 o mesmo comportamento da Figura 4.8, ou seja, devido ao processo de solidificação do material, a energia necessária para crescimento de trinca tende a diminuir. Observa-se ainda que o desvio padrão apresenta-se com alta magnitude e, desta 
forma, uma alta variabilidade dos dados de saída de $G_{I}$, dificulta a verificação dos modelos.

\subsection{1. $G_{I}$ para $t c=1$ dia $\mathrm{e} t=11$ dias}

O primeiro caso estudado consiste em $t_{c}=1$ dia e $t=11$ dias, sendo considerado a construção de meta modelos para variáveis aleatórias com regressão $P 2, R B F$ e $R B F P 1$. Além disso, verifica-se a seguir a Tabela 4.5 para regressão polinomial e seus respectivos parâmetros $S S E, r^{2}$ e RMSE, sendo $N C$ o número de coeficientes e $\mathrm{N}$ o número de amostras utilizadas para determinação dos coeficientes.

Tabela 4.5 - Parâmetros de P2 para $t_{c}=1$ dia e $t=11$ dias

\begin{tabular}{ccccccc}
\hline \multirow{2}{*}{ Cenários } & $\begin{array}{c}\text { Regressão } \\
G_{I}\end{array}$ & SSE & RMSE & $\mathrm{r}^{2}$ & $\mathrm{~N}$ & \multirow{2}{*}{$\mathrm{NC}$} \\
& $L H S$ & $2,31 \mathrm{E}+3$ & 5,41 & 0,99 & 79 & 78 \\
\multirow{2}{*}{$C_{I}$} & $S S$ & $3,74 \mathrm{E}+3$ & 6,88 & 0,99 & 79 & 78 \\
& $L H S$ & $2,30 \mathrm{E}+2$ & 1,71 & 0,99 & 79 & 78 \\
\multirow{2}{*}{$C_{I I}$} & $S S$ & $1,91 \mathrm{E}+3$ & 4,91 & 0,99 & 79 & 78 \\
& $L H S$ & $1,40 \mathrm{E}+4$ & 13,31 & 0,99 & 79 & 78 \\
\multirow{2}{*}{$C_{I I I}$} & $S S$ & $5,43 \mathrm{E}+2$ & 2,62 & 0,99 & 79 & 78 \\
\hline \multirow{2}{*}{$C_{I}$} & $L H S$ & $5,94 \mathrm{E}+5$ & 54,52 & 0,90 & 200 & 78 \\
& $S S$ & $1,72 \mathrm{E}+6$ & 92,82 & 0,81 & 200 & 78 \\
\multirow{2}{*}{$C_{I I}$} & $L H S$ & $5,50 \mathrm{E}+5$ & 52,45 & 0,91 & 200 & 78 \\
& $S S$ & $6,86 \mathrm{E}+5$ & 58,56 & 0,91 & 200 & 78 \\
\hline
\end{tabular}

$\mathrm{Na}$ Tabela 4.5, nota-se que o menor somatório de erros $(S S E)$ encontra-se para $\mathrm{N}=79$. No caso do RMSE, percebe-se que o mesmo se assemelha ao SSE, ou seja, os mesmos se apresentam muito inferiores que para valores de $\mathrm{N}$ igual a 200 amostras. Por fim, para $r^{2}$, nota-se que para $N=79$, mais de $95 \%$ da variabilidade dos pontos pode ser explicada pela regressão, ou seja, o meta modelo regredido consegue reproduzir adequadamente os dados de entrada. Contudo, para $N=200$, o mesmo não acontece, pois os valores de SSE e RMSE são bastante elevados de forma a dificultar a validação do modelo. Percebe-se ainda na Tabela 4.5 que à medida que $N$ aumenta, a magnitude de $r^{2}$ diminui. Assim, para valores altos de N, o modelo polinomial tende a apresentar dificuldade em adequar-se. Na Figura 4.9 apresentam-se as respectivas $P D F s$ de $P 2$ para $L H S$ e $S S$ dos cenários $C_{I}$ e $C_{I I}$. 


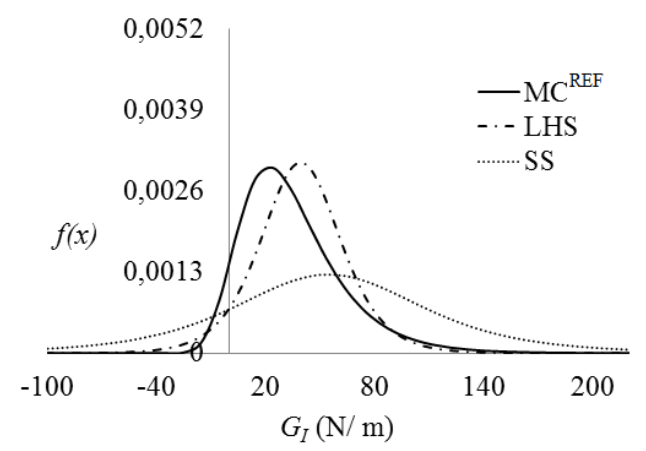

(a) $\mathrm{C}_{\mathrm{I}}, \mathrm{N}=79$

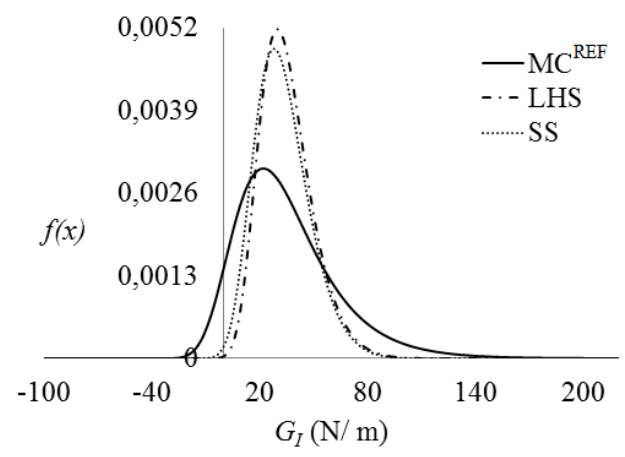

(c) $\mathrm{C}_{\mathrm{I}}, \mathrm{N}=200$

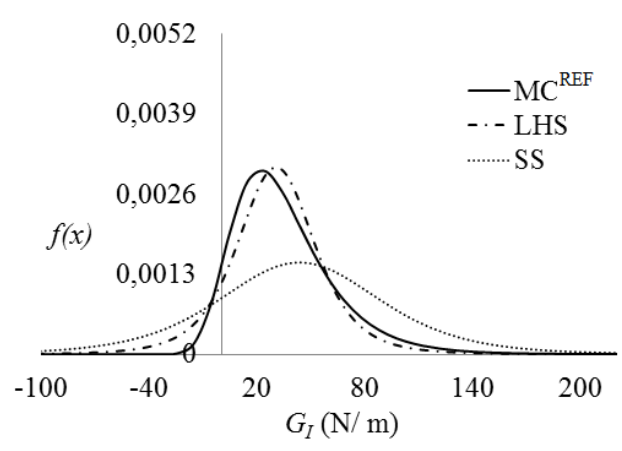

(b) $\mathrm{C}_{\mathrm{II}}, \mathrm{N}=79$

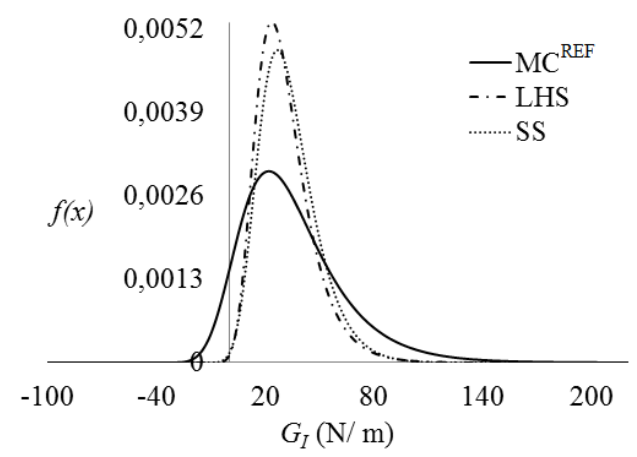

(d) $\mathrm{C}_{\mathrm{II}}, \mathrm{N}=200$

Figura 4.9 - PDFs de $G_{I}$ sendo (a) e (b) com N =79 e (c) e (d) com N = 200 para $P 2 \operatorname{com} t_{c}$ $=1$ dia e $t=11$ dias.

Na Figura 4.9, cada modelo considerou-se 5E+5 simulações para MC. A validação das PDFs é realizada através da comparação dos modelos com os resultados de MC para 5E+5 simulações de $M C^{R E F}$. Outra análise consiste no NRMSE dado pela Tabela 4.6.

Tabela 4.6 - Ajuste de $G_{I}$ para $P 2 \operatorname{com} t_{c}=1$ dia e $t=11$ dias

\begin{tabular}{c|c:c|c}
\hline \multirow{2}{*}{ Cenário } & Amostras & $\mathrm{N}$ & NRMSE \\
\hline$M C^{R E F}$ & - & - & 1,00 \\
\hline \multirow{2}{*}{$C_{I}$} & $L H S$ & 79 & $-0,08$ \\
& $S S$ & 79 & $-1,50$ \\
$C_{I I}$ & $L H S$ & 79 & $-0,26$ \\
& $S S$ & 79 & $-1,01$ \\
\hline \multirow{2}{*}{$C_{I I I}$} & $L H S$ & 79 & $-3,32$ \\
& $S S$ & 79 & $-5,44$ \\
\hline \multirow{2}{*}{$C_{I}$} & $L H S$ & 200 & $-0,10$ \\
& $S S$ & 200 & $-0,11$ \\
\hline \multirow{2}{*}{$C_{I I}$} & $L H S$ & 200 & $-0,12$ \\
& $S S$ & 200 & $-0,12$ \\
\hline
\end{tabular}

Na Tabela 4.6 nota-se que $C_{I}$ e $C_{I I}$ consistem nos melhores resultados para $N=200$. Já o cenário $C_{I I I}$ não se comporta bem, pois o mesmo apresenta-se muito disperso quando comparado a $M C^{R E F}$. Na Tabela 4.7 apresentam-se os parâmetros para $R B F$. 
Tabela 4.7 - Parâmetros de $R B F$ para $t_{c}=1$ dia e $t=11$ dias

\begin{tabular}{ccccccc}
\hline \multirow{2}{*}{ Cenários } & $\begin{array}{c}\text { Regressão } \\
G_{I}\end{array}$ & SSE & RMSE & $\mathrm{r}^{2}$ & \multirow{2}{*}{$\mathrm{N}$} & \multirow{2}{*}{$\mathrm{NC}$} \\
\hline \multirow{2}{*}{$C_{I}$} & $L H S$ & $1,30 \mathrm{E}-23$ & $4,10 \mathrm{E}-13$ & 1,00 & 79 & 79 \\
& $S S$ & $1,04 \mathrm{E}-23$ & $3,62 \mathrm{E}-13$ & 1,00 & 79 & 79 \\
\multirow{2}{*}{$C_{I I}$} & $L H S$ & $5,80 \mathrm{E}-24$ & $2,71 \mathrm{E}-13$ & 1,00 & 79 & 79 \\
& $S S$ & $1,38 \mathrm{E}-20$ & $1,32 \mathrm{E}-11$ & 1,00 & 79 & 79 \\
\multirow{2}{*}{$C_{I I I}$} & $L H S$ & $3,81 \mathrm{E}-23$ & $6,95 \mathrm{E}-13$ & 1,00 & 79 & 79 \\
& $S S$ & $6,16 \mathrm{E}-24$ & $2,79 \mathrm{E}-13$ & 1,00 & 79 & 79 \\
\hline \multirow{2}{*}{$C_{I}$} & $L H S$ & $1,15 \mathrm{E}-21$ & $2,40 \mathrm{E}-12$ & 1,00 & 200 & 200 \\
& $S S$ & $5,39 \mathrm{E}-22$ & $1,64 \mathrm{E}-12$ & 1,00 & 200 & 200 \\
\multirow{2}{*}{$C_{I I}$} & $L H S$ & $3,03 \mathrm{E}-22$ & $1,23 \mathrm{E}-12$ & 1,00 & 200 & 200 \\
& $S S$ & $3,34 \mathrm{E}-22$ & $1,29 \mathrm{E}-12$ & 1,00 & 200 & 200 \\
\hline
\end{tabular}

Na Tabela 4.7 observa-se o processo de interpolação por $R B F$ para os cenários $C_{I}$ a $C_{I I I}$ para $\theta$ da Tabela 4.5. De acordo com a Tabela 4.7, a somatória de erros apresenta-se com valores praticamente nulos, pois o processo de interpolação passa exatamente nos pontos da amostragem. Nota-se ainda que o coeficiente de determinação apresenta-se igual a 1 independente do cenário, da amostragem ou do valor de N. Na Tabela 4.8 encontra-se NRMSE para as RBFs do primeiro $\theta$.

Tabela 4.8 - Ajuste de $G_{I}$ para $R B F \operatorname{com} t_{c}=1$ dia e $t=11$ dias

\begin{tabular}{c|c|c|c}
\hline \multirow{2}{*}{ Cenário } & Amostras & $\mathrm{N}$ & NRMSE \\
\hline$M C^{R E F}$ & - & - & 1,00 \\
\hline \multirow{2}{*}{$C_{I}$} & $L H S$ & 79 & 0,01 \\
& $S S$ & 79 & $-0,54$ \\
\hline$C_{I I}$ & $L H S$ & 79 & $-0,18$ \\
& $S S$ & 79 & 0,01 \\
$C_{I I I}$ & $L H S$ & 79 & $-1,19$ \\
& $S S$ & 79 & $-0,28$ \\
\hline \multirow{2}{*}{$C_{I}$} & $L H S$ & 200 & $-0,39$ \\
$C_{I I}$ & $S S$ & 200 & $-0,55$ \\
\hline
\end{tabular}

Observa-se na Tabela 4.8 que os resultados do ajuste à $M C^{R E F}$ são bons para o cenário $C_{I I}$. Além disso, nota-se que $C_{I I I}$ apresenta-se com o pior comportamento mesmo tendo seu ajuste melhorado quando comparado a $P 2$. Por fim, conclui-se que a tabela apresenta meta modelos que podem validar o fenômeno $G_{I}$. Na Figura 4.10, observa-se as respectivas PDFs da Tabela 4.7 para cenários $C_{I}$ e $C_{I I}$. As Figura 4.10a e 4.10b apresentam valores de $N=79$ e as Figuras 4.10 c e $4.10 \mathrm{~d}$ com $N=200$, tendo todos os meta modelos, valores do suporte $\delta=1$. 


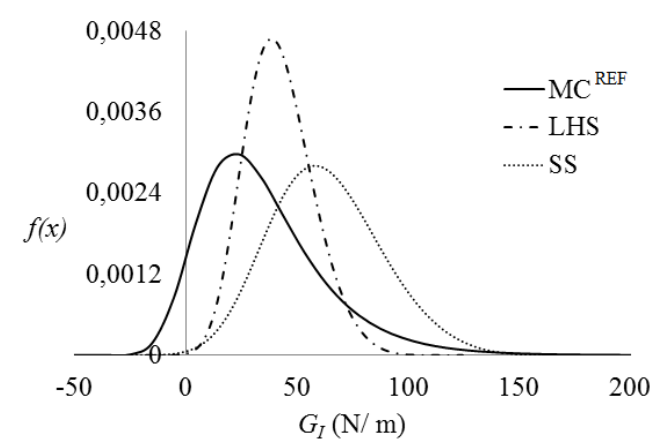

(a) $\mathrm{C}_{\mathrm{I}}, \mathrm{N}=79$

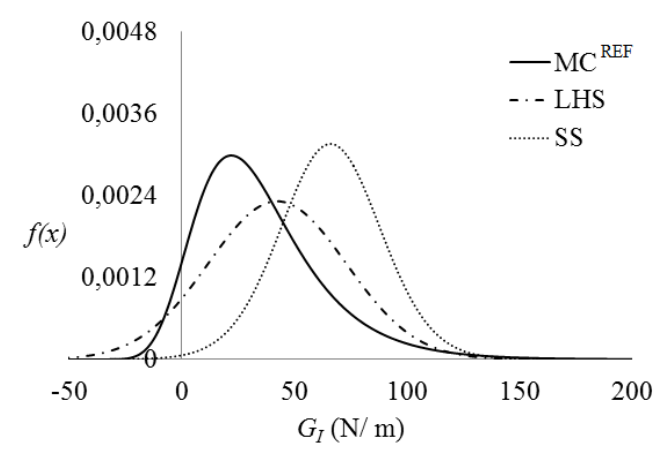

(c) $\mathrm{C}_{\mathrm{I}}, \mathrm{N}=200$

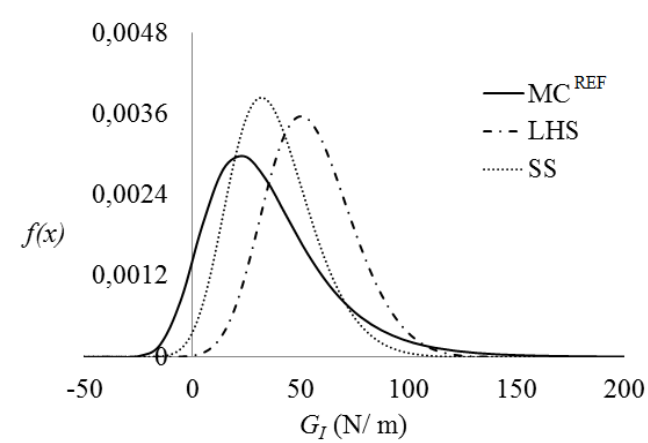

(b) $\mathrm{C}_{\mathrm{II}}, \mathrm{N}=79$

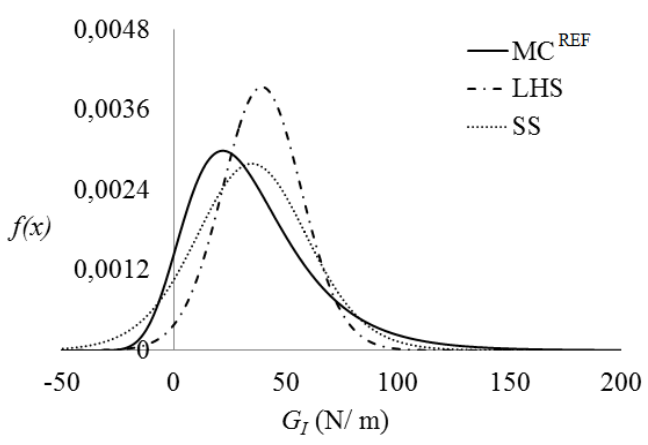

(d) $\mathrm{C}_{\mathrm{II}}, \mathrm{N}=200$

Figura 4.10 - PDFs de $G_{I}$ sendo (a) e (b) com N = 79 e (c) e (d) com N = 200 para RBF $\operatorname{com~}_{\mathrm{c}}=1$ dia e $\mathrm{t}=11$ dias.

Na Figura 4.10, nota-se que as PDFs apresentam-se com um melhor comportamento em relação à $M C^{R E F}$ que o meta modelo $P 2$. Contudo, o melhor resultado encontra-se para $C_{I I}$ com $N=200$, pois o mesmo tende a se aderir quase por completo ao $M C^{R E F}$. A interpolação RBFPl consiste no ultimo meta modelo para o primeiro caso de $\theta$ apresentando resultados na Tabela $4.9 \operatorname{com} \delta=1$.

Tabela 4.9 - Parâmetros de RBFP1 para $t_{c}=1$ dia e $t=11$ dias

\begin{tabular}{ccccccc}
\hline \multirow{2}{*}{ Cenários } & $\begin{array}{c}\text { Regressão } \\
G_{I}\end{array}$ & SSE & RMSE & $\mathrm{r}^{2}$ & $\mathrm{~N}$ & \multirow{2}{*}{$\mathrm{NC}$} \\
& $L H S$ & $1,97 \mathrm{E}-21$ & $4,99 \mathrm{E}-12$ & 1,00 & 79 & 79 \\
\multirow{2}{*}{$C_{I}$} & $S S$ & $3,65 \mathrm{E}-22$ & $2,15 \mathrm{E}-12$ & 1,00 & 79 & 79 \\
& $L H S$ & $8,19 \mathrm{E}-22$ & $3,22 \mathrm{E}-12$ & 1,00 & 79 & 79 \\
\multirow{2}{*}{$C_{I I}$} & $S S$ & $5,14 \mathrm{E}-22$ & $2,55 \mathrm{E}-12$ & 1,00 & 79 & 79 \\
& $L H S$ & $2,36 \mathrm{E}-21$ & $5,47 \mathrm{E}-12$ & 1,00 & 79 & 79 \\
\multirow{2}{*}{$C_{I I I}$} & $S S$ & $1,20 \mathrm{E}-22$ & $1,23 \mathrm{E}-12$ & 1,00 & 79 & 79 \\
\hline \multirow{2}{*}{$C_{I}$} & $L H S$ & $2,08 \mathrm{E}-19$ & $3,22 \mathrm{E}-11$ & 1,00 & 200 & 200 \\
& $S S$ & $7,49 \mathrm{E}-17$ & $6,12 \mathrm{E}-10$ & 1,00 & 200 & 200 \\
\multirow{2}{*}{$C_{I I}$} & $L H S$ & $3,92 \mathrm{E}-20$ & $1,40 \mathrm{E}-11$ & 1,00 & 200 & 200 \\
& $S S$ & $1,46 \mathrm{E}-18$ & $8,54 \mathrm{E}-11$ & 1,00 & 200 & 200 \\
\hline
\end{tabular}

Nota-se na Tabela 4.9 o comportamento semelhante ao empregar-se apenas $R B F$, onde todos os parâmetros de análise apresentam-se muito superiores ao P2. Já na Tabela 4.10 apresenta-se $N R M S E$ para $R B F P 1$ para o primeiro caso de $\theta$. 
Tabela 4.10 - Ajuste de $G_{I}$ para $R B F P 1 \operatorname{com} t_{c}=1$ dia e $t=11$ dias

\begin{tabular}{c|c|c|c}
\hline \multirow{2}{*}{ Cenário } & Amostras & $\mathrm{N}$ & NRMSE \\
\hline$M C^{\text {REF }}$ & - & - & 1,00 \\
\hline \multirow{2}{*}{$C_{I}$} & $L H S$ & 79 & $-0,67$ \\
& $S S$ & 79 & $-0,13$ \\
\hline \multirow{2}{*}{$C_{I I}$} & $L H S$ & 79 & 0,07 \\
& $S S$ & 79 & $-0,12$ \\
\hline \multirow{2}{*}{$C_{I I I}$} & $L H S$ & 79 & $-1,65$ \\
& $S S$ & 79 & $-0,19$ \\
\hline \multirow{2}{*}{$C_{I}$} & $L H S$ & 200 & $-0,13$ \\
& $S S$ & 200 & $-0,96$ \\
\hline \multirow{2}{*}{$C_{I I}$} & $L H S$ & 200 & $-0,21$ \\
& $S S$ & 200 & $-0,12$ \\
\hline
\end{tabular}

Na Tabela 4.10 observa-se novamente um melhor comportamento dos cenários $C_{I}$ e $C_{I I} \mathrm{e}$ um pior comportamento para $C_{I I I}$. Além disso, na Figura 4.11 as respectivas funções densidade de probabilidade são apresentadas para um numero de $5 \mathrm{E}+5$ simulações. Na Figura $4.11 \mathrm{a}$ e $4.11 \mathrm{~b}$ observa-se os cenários da Tabela 4.5 para $N=79$ sendo ambos retratados para amostragens aleatórias. Já na Figura 4.11c e 4.11d nota-se cenários com $N=200$ sendo também adotadas amostragens de $L H S$ e $S S$.

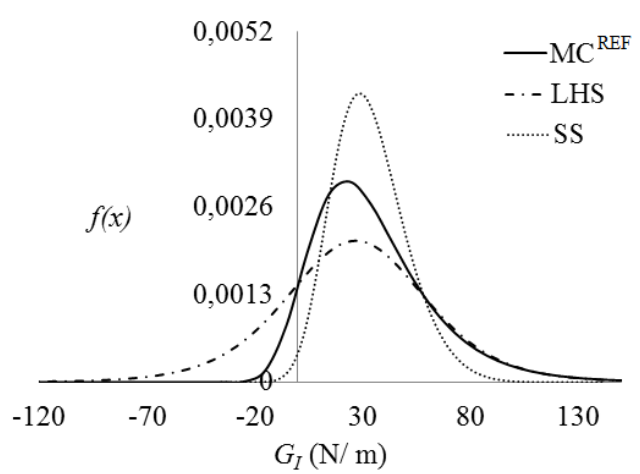

(a) $\mathrm{C}_{\mathrm{I}}, \mathrm{N}=79$

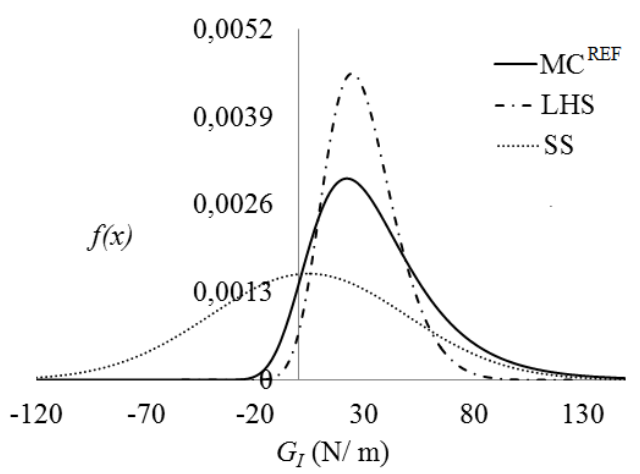

(c) $\mathrm{C}_{\mathrm{I}}, \mathrm{N}=200$

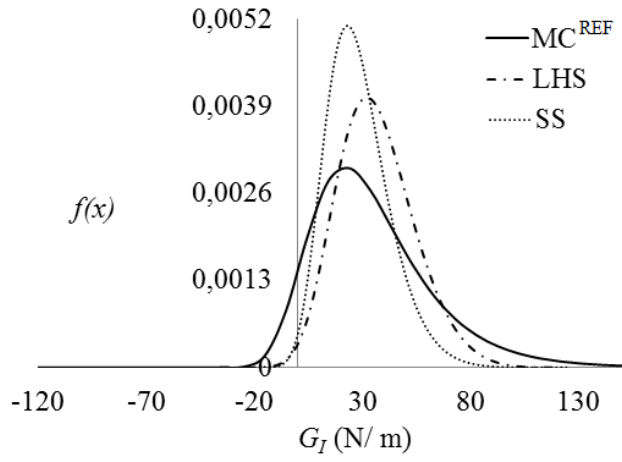

(b) $\mathrm{C}_{\mathrm{II}}, \mathrm{N}=79$

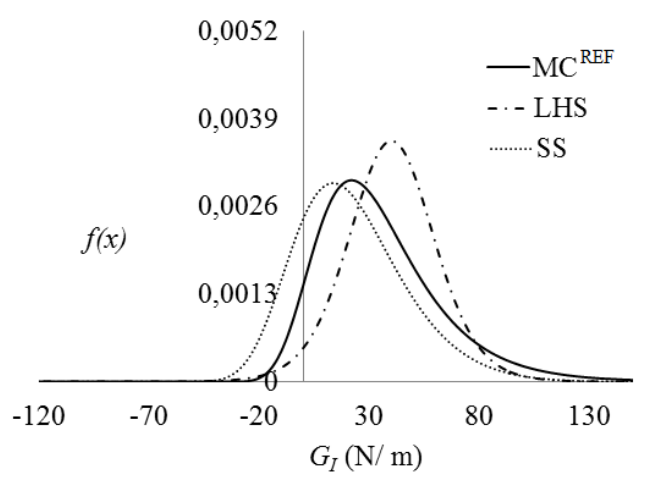

(d) $\mathrm{C}_{\mathrm{II}}, \mathrm{N}=200$

Figura 4.11 - $P D F s$ de $G_{I}$ sendo (a) e (b) com $\mathrm{N}=79$ e (c) e (d) com N = 200 para RBFP1 com $\mathrm{t}_{\mathrm{c}}=1$ dia e $\mathrm{t}=11$ dias. 
Na Figura 4.11a, nota-se que para o cenário $C_{I}$ a amostragem por $L H S$ tende a aderir-se melhor ao $M C^{R E F}$. Na Figura $4.11 \mathrm{~b}$, o cenário a ser considerado é o $C_{I I}$ sendo também analisado para amostragens semelhantes à Figura 4.11a. Contudo, os resultados são mais adequados para $C_{I}$. Na Figura $4.11 \mathrm{c}$ a amostragem por SS apresenta-se com alto nível de dispersão comparado ao apresentado pelas Figura 4.9c e 4.10c. Por fim, na Figura 4.11d observa-se uma melhor adesão dos resultados em relação ao $\mathrm{MC}^{\mathrm{REF}}$. Desta forma, concluise ainda que a adição do polinômio de primeira ordem P1 ao meta modelo de função de base radial tende a apresentar uma comportamento superior comparado aos outros meta modelos para um tempo de carregamento $t_{c}=1$ dias e um tempo de análise do concreto $t=$ 11 dias. Na Figura 4.12 apresenta-se a única análise de $C_{I I I}$ para o primeiro caso de $\theta$.

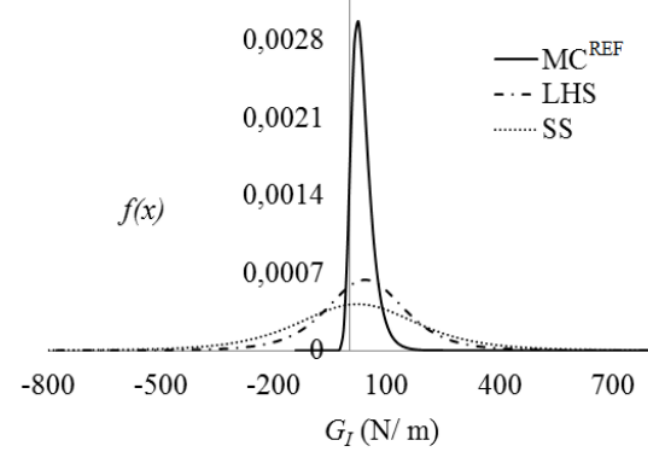

(a) $\mathrm{C}_{\mathrm{III}}, \mathrm{P} 2$

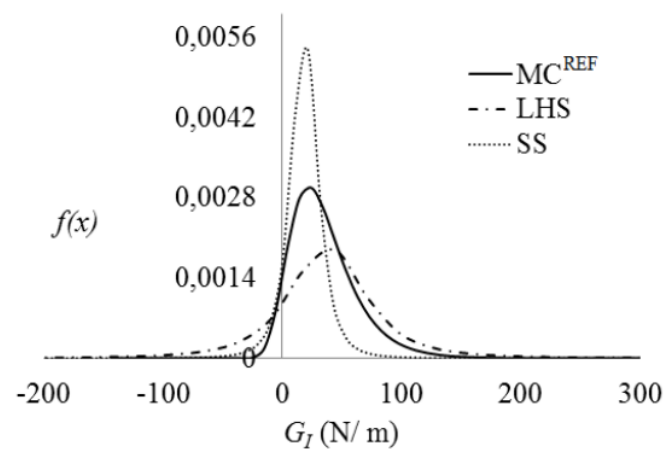

(b) $\mathrm{C}_{\mathrm{III}}, \mathrm{RBF}$

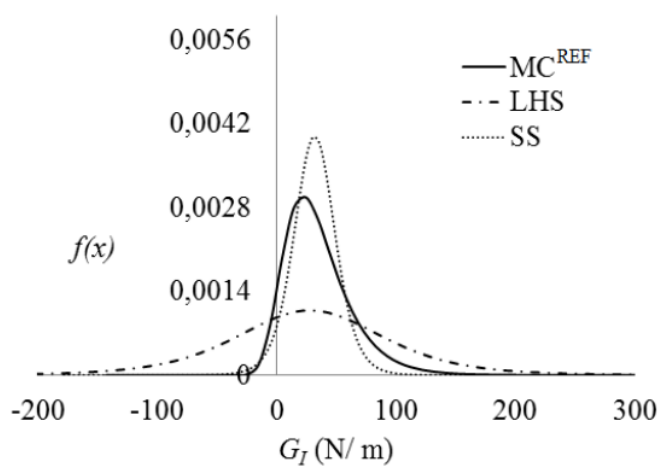

(c) $\mathrm{C}_{\mathrm{III}}, \mathrm{RBFP} 1$

Figura $4.12-P D F s$ de $G_{I}$ para $C_{I I I} \operatorname{com} N=79$ e para $\mathrm{t}_{\mathrm{c}}=1$ dia e $\mathrm{t}=11$ dias.

Observa-se na Figura 4.12 que o comportamento de $C_{I I I}$ apresenta-se com alta dispersão de dados, sendo portanto, descartado sua análise nas próximas seções.

\subsubsection{G $_{\text {I }}$ para $\mathrm{tc}=7$ dias $\mathrm{e} t=17$ dias}

Um segundo caso estudado consiste em $t_{c}=7$ dia e $t=17$ dias, sendo considerado a construção de meta modelos para variáveis aleatórias. Os modelos apresentados consistem apenas as $P D F s$, pois o comportamento dos parâmetros de regressão é semelhante ao do 
primeiro caso. Para verificação de $S S E, R M S E$ e $r^{2}$ é necessário consultar o apêndice A. Na Tabela 4.11 apresenta-se NRMSE das distribuições para $P 2$.

Tabela 4.11 - Ajuste de $G_{I}$ para $P 2 \operatorname{com} t_{c}=7$ dia e $t=17$ dias

\begin{tabular}{c|c|c|c}
\hline \multirow{2}{*}{ Cenário } & \multirow{2}{*}{ Amostras } & $\mathrm{N}$ & NRMSE \\
\hline$M C^{R E F}$ & - & - & 1,00 \\
\hline \multirow{2}{*}{$C_{I}$} & $L H S$ & 79 & $-1,24$ \\
& $S S$ & 79 & $-0,29$ \\
\hline \multirow{2}{*}{$C_{I I}$} & $L H S$ & 79 & $-0,39$ \\
& $S S$ & 79 & $-0,53$ \\
\hline \multirow{2}{*}{$C_{I}$} & $L H S$ & 200 & $-0,09$ \\
& $S S$ & 200 & $-0,09$ \\
\multirow{2}{*}{$C_{I I}$} & $L H S$ & 200 & $-0,11$ \\
& $S S$ & 200 & $-0,09$ \\
\hline
\end{tabular}

Observa-se na Tabela 4.11 o ajuste de $C_{I I}$ como o melhor para $P 2$. Percebe-se ainda que os resultados de $P 2$ são melhores que os de $\theta$ anteriores. Para a quantificação da incerteza, PDFs são apresentadas na Figura 4.13.

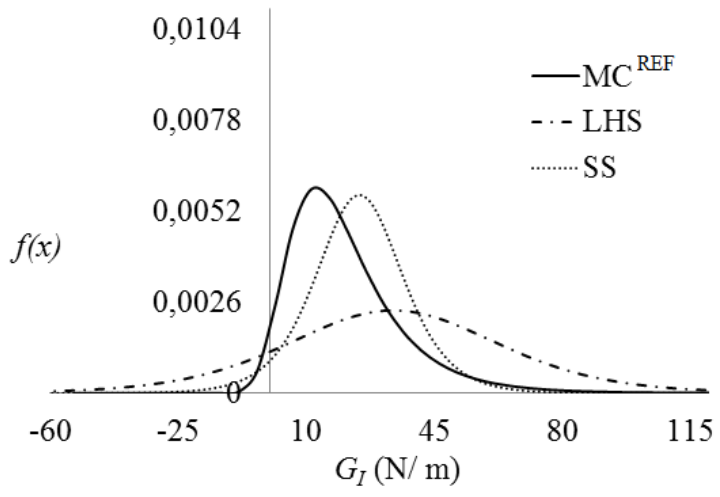

(a) $\mathrm{C}_{\mathrm{I}}, \mathrm{N}=79$

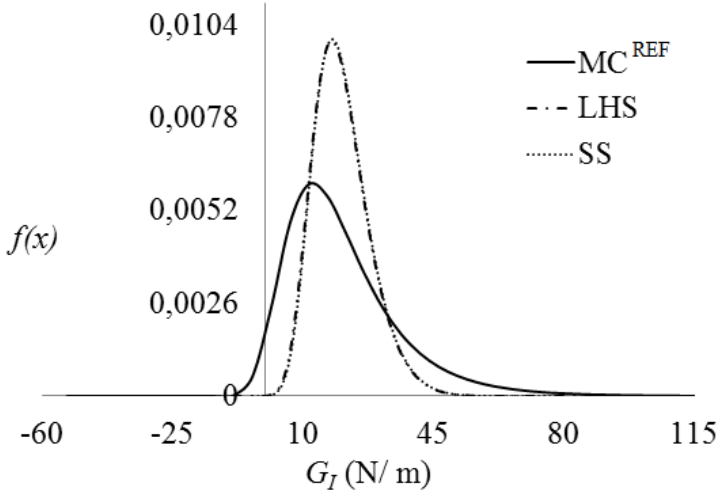

(c) $\mathrm{C}_{\mathrm{I}}, \mathrm{N}=200$

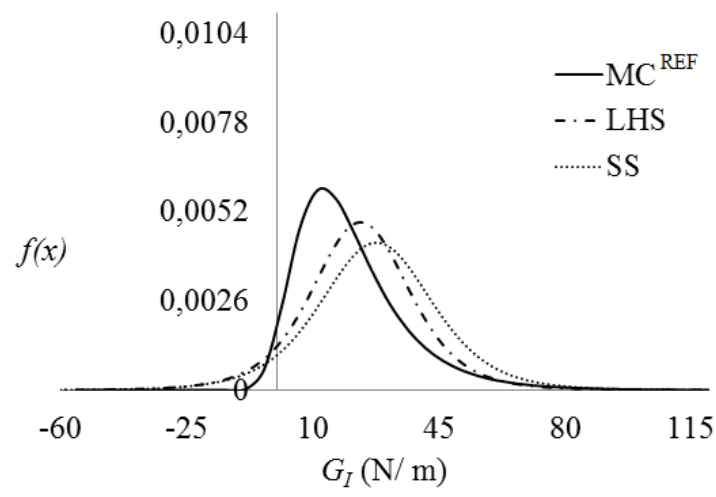

(b) $\mathrm{C}_{\mathrm{II}}, \mathrm{N}=79$

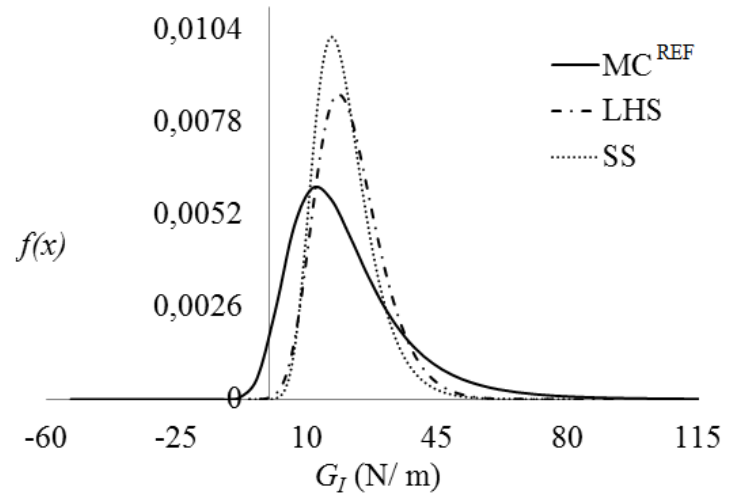

(d) $\mathrm{C}_{\mathrm{II}}, \mathrm{N}=200$

Figura 4.13 - $P D F s$ de $G_{I}$ sendo (a) e (b) $\operatorname{com} N=79$ e (c) e (d) $\operatorname{com} N=200$ para $P 2$ com

$$
\mathrm{t}_{\mathrm{c}}=7 \text { dia e } \mathrm{t}=17 \text { dias. }
$$

De acordo com a Figura 4.13, as $P D F s$ para $N=200$ exibem melhores resultados comparados com $N=79$. Além disso, pode-se inferir para o segundo caso de $\theta$ que o 
modelo que mais se adere consiste no cenário $C_{I I}$. Na Tabela 4.12 apresenta-se $N R M S E$ para as $R B F$ s do segundo caso de $\theta$.

Tabela 4.12 - Ajuste de $G_{I}$ para $R B F \operatorname{com} t_{c}=7$ dia e $t=17$ dias

\begin{tabular}{c|c|c|c}
\hline \multirow{2}{*}{ Cenário } & \multirow{2}{*}{ Amostras } & $\mathrm{N}$ & NRMSE \\
\hline$M C^{R E F}$ & - & - & 1,00 \\
\hline \multirow{2}{*}{$C_{I}$} & $L H S$ & 79 & $-1,92$ \\
& $S S$ & 79 & $-3,28$ \\
\hline \multirow{2}{*}{$C_{I I}$} & $L H S$ & 79 & $-3,63$ \\
& $S S$ & 79 & $-1,09$ \\
\hline \multirow{2}{*}{$C_{I}$} & $L H S$ & 200 & 0,03 \\
& $S S$ & 200 & 0,03 \\
\multirow{2}{*}{$C_{I I}$} & $L H S$ & 200 & 0,05 \\
& $S S$ & 200 & $-0,15$ \\
\hline
\end{tabular}

Nota-se na Tabela 4.12 que para $N=79$ as distribuições encontram-se dispersas quando comparadas a $M C^{R E F}$. Já para $N=200$ o ajuste é considerado adequado, pois os valores estão mais próximos de 1. Na Figura 4.14 as PDFs do meta modelo são comparadas a $\mathrm{MC}^{\mathrm{REF}}$ com $5 \mathrm{E}+5$ simulações.

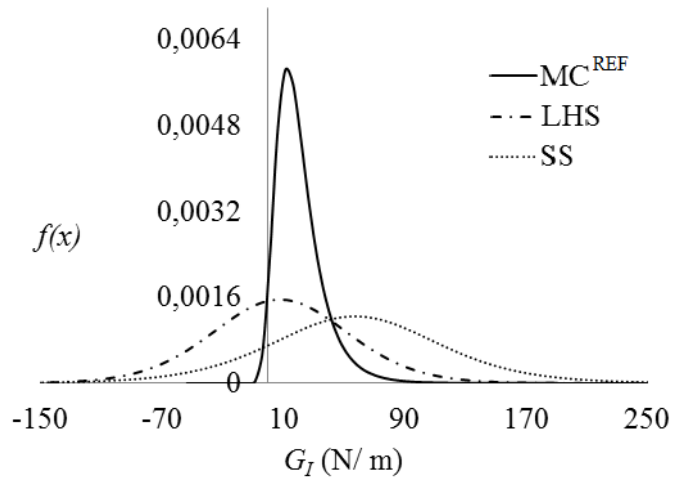

(a) $\mathrm{C}_{\mathrm{T}}, \mathrm{N}=79$

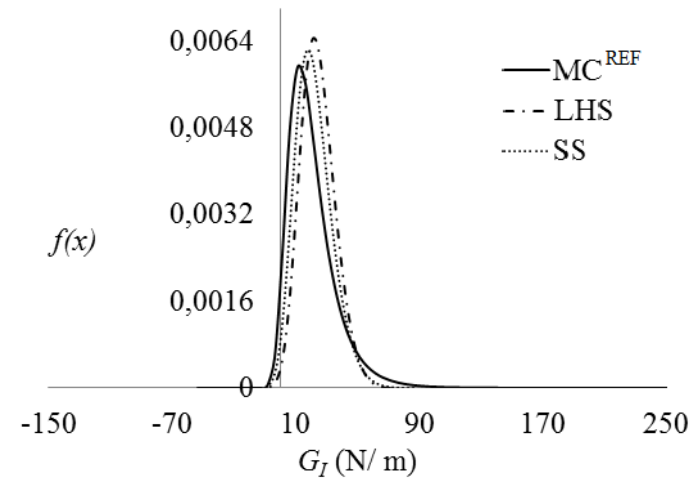

(c) $\mathrm{C}_{\mathrm{I}}, \mathrm{N}=200$

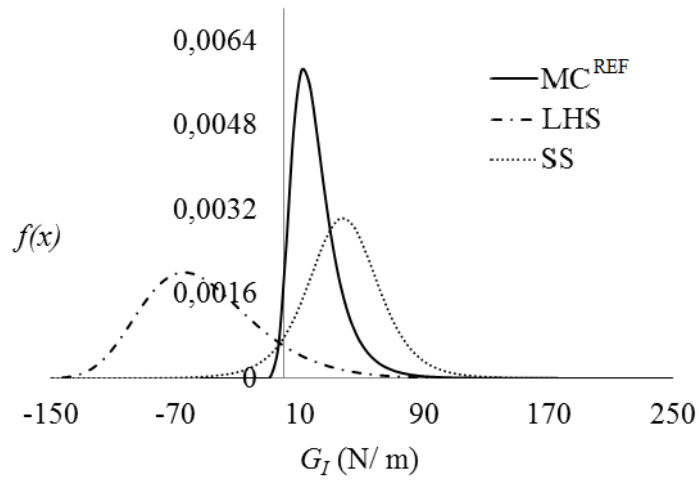

(b) $\mathrm{C}_{\mathrm{T}}, \mathrm{N}=79$

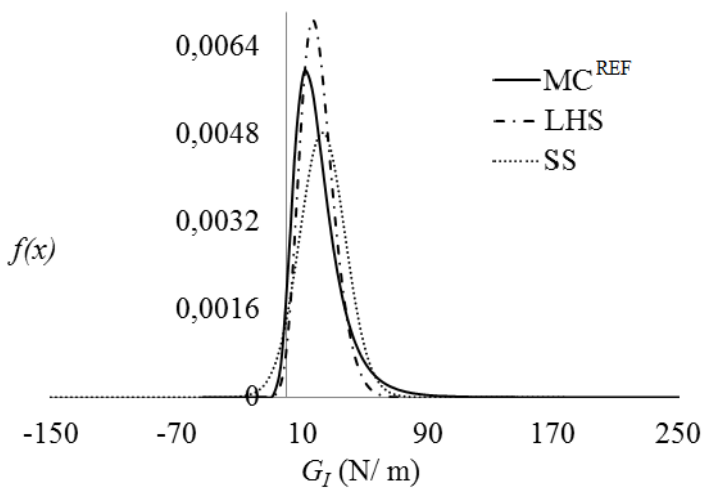

(d) $\mathrm{C}_{\mathrm{II}}, \mathrm{N}=200$

Figura 4.14 - PDFs de $G_{I}$ sendo (a) e (b) $\operatorname{com} N=79$ e (c) e (d) $\operatorname{com} N=200$ para $R B F$ com $t_{c}=7$ dia e $t=17$ dias. 
Nota-se na Figura 4.14 que para $N=79$, encontra-se com alto nível de dispersão. Percebe-se ainda que $N=200$ apresenta bons resultados tanto para $C_{I}$ quanto $C_{I I}$. Já na Tabela 4.13 encontram-se os NRMSE para o segundo valor de $\theta$.

Tabela 4.13 - Ajuste de $G_{I}$ para $R B F P 1$ com $t_{c}=7$ dia e $t=17$ dias

\begin{tabular}{c|c|c|c}
\hline \multirow{2}{*}{ Cenário } & Amostras & $\mathrm{N}$ & NRMSE \\
\hline$M C^{R E F}$ & - & - & 1,00 \\
\hline \multirow{2}{*}{$C_{I}$} & $L H S$ & 79 & $-0,15$ \\
& $S S$ & 79 & 0,03 \\
\hline \multirow{2}{*}{$C_{I I}$} & $L H S$ & 79 & $-0,10$ \\
& $S S$ & 79 & $-0,12$ \\
\hline \multirow{2}{*}{$C_{I}$} & $L H S$ & 200 & $-0,15$ \\
& $S S$ & 200 & 0,08 \\
\hline \multirow{2}{*}{$C_{I I}$} & $L H S$ & 200 & 0,08 \\
& $S S$ & 200 & 0,09 \\
\hline
\end{tabular}

Na Tabela 4.13 observa-se um melhor ajuste quando comparado a $R B F$ e a $P 2$. Na Figura 4.15, diferentes $P D F s$ são apresentadas para $5 \mathrm{E}+5$ simulações de $\mathrm{MC}^{\mathrm{REF}}$ para os cenários $C_{I}$ e $C_{I I}$.

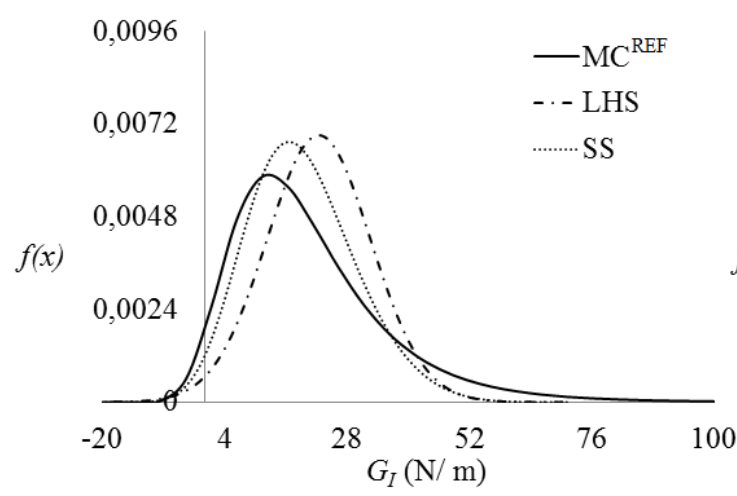

(a) $\mathrm{C}_{\mathrm{I}}, \mathrm{N}=79$

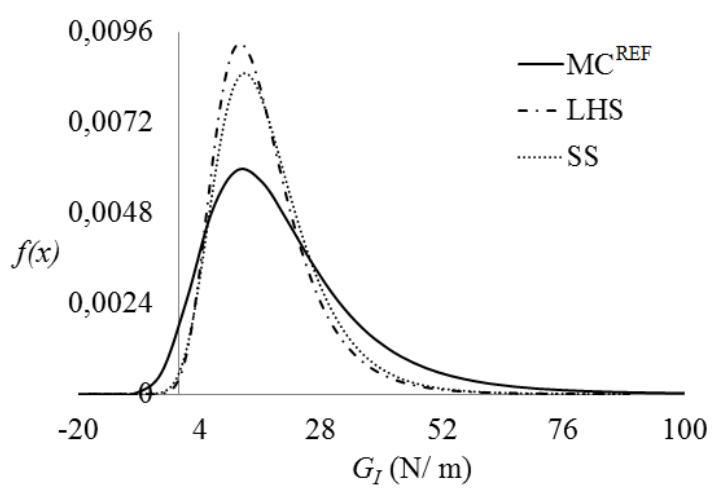

(c) $\mathrm{C}_{\mathrm{I}}, \mathrm{N}=200$

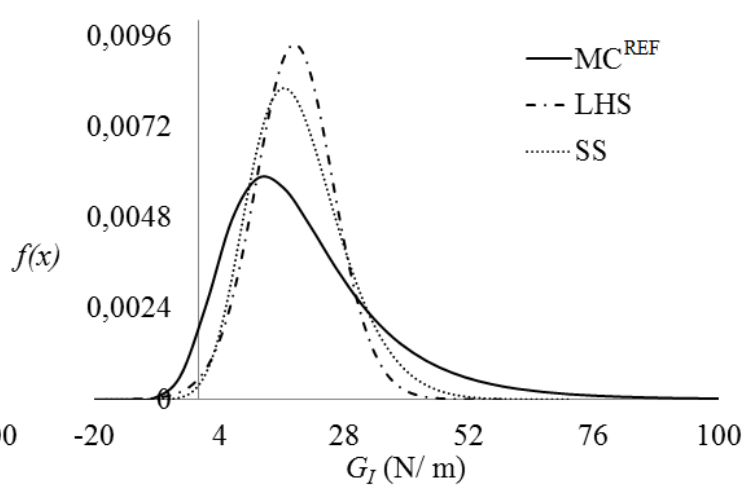

(b) $\mathrm{C}_{\mathrm{II}}, \mathrm{N}=79$

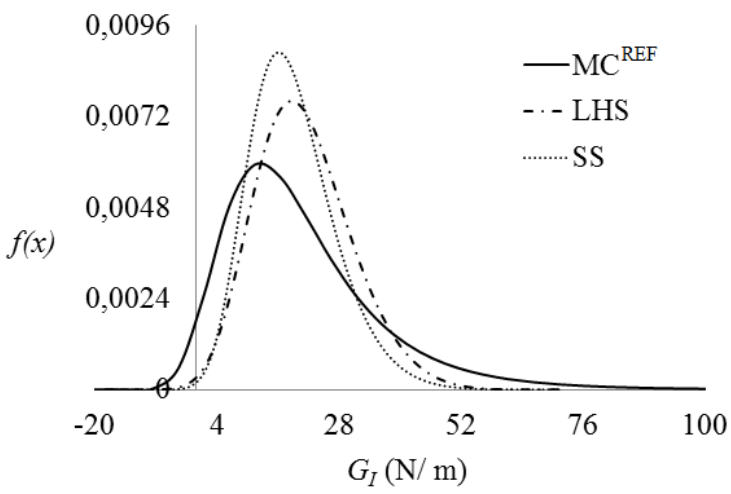

(d) $\mathrm{C}_{\mathrm{II}}, \mathrm{N}=200$

Figura 4.15 - PDFs de $G_{I}$ sendo (a) e (b) com $N=79$ e (c) e (d) com $N=200$ para $R B F P I$ com $t_{c}=7$ dia e $t=17$ dias. 
Na Figura 4.15 percebe que ambas as amostragens tendem a se aderir melhor ao cenário $C_{I}$. Já para o $C_{I I}$, o mesmo acontece, mas com uma adesão das $P D F s$ inferior ao da Figura 4.15a-c.

\subsection{3. $\mathrm{G}_{\mathrm{I}}$ para $\mathrm{tc}=14$ dias $\mathrm{e} t=24$ dias}

No último caso de análise de $G_{I}$, o tempo de carregamento $t_{c}=14$ dias e o tempo de análise $t=24$ dias, sendo os parâmetros de análise o SSE, RMSE e $r^{2}$ dados no apêndice B. Na Figura 4.16 apresentam-se as $P D F s$ para $\mathrm{P} 2$ para os cenários $C_{I}$ e $C_{I I}$.

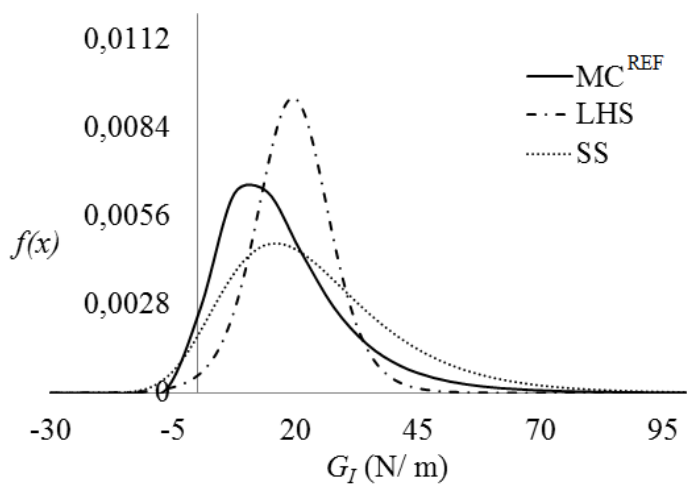

(a) $\mathrm{C}_{\mathrm{I}}, \mathrm{N}=79$

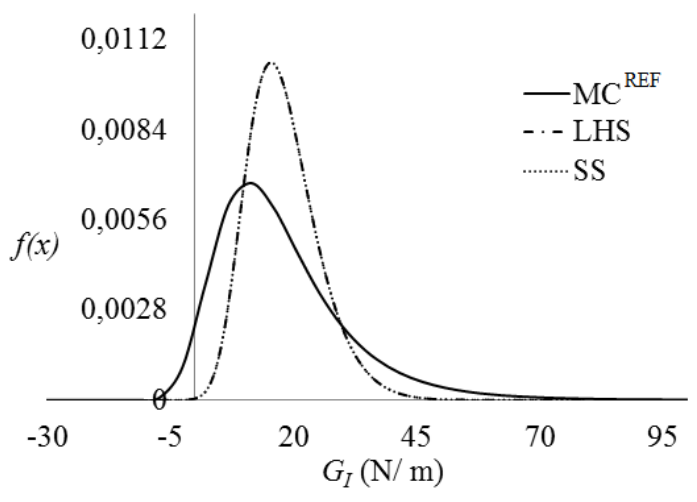

(c) $\mathrm{C}_{\mathrm{I}}, \mathrm{N}=200$

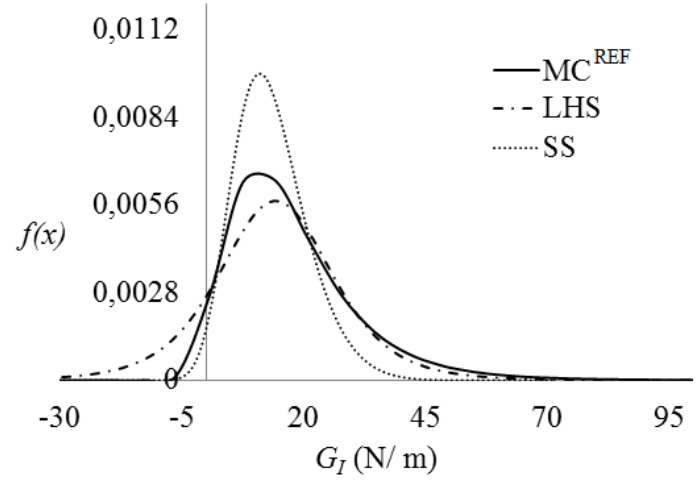

(b) $\mathrm{C}_{\mathrm{II}}, \mathrm{N}=79$

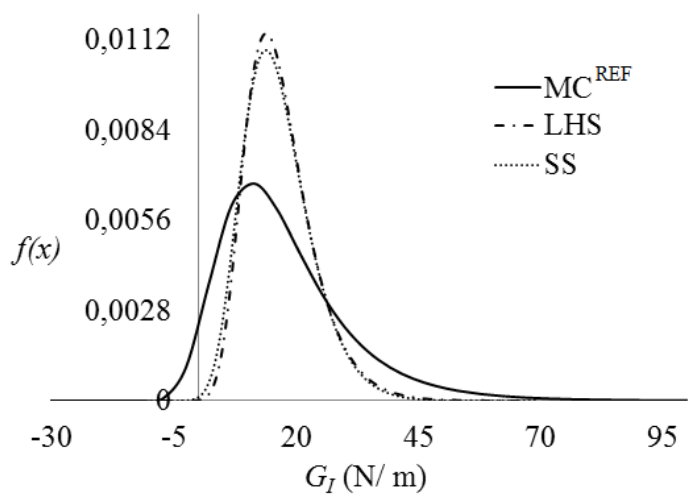

(d) $\mathrm{C}_{\mathrm{II}}, \mathrm{N}=200$

Figura 4.16 - PDFs de $G_{I} \operatorname{sendo}($ a) e (b) $\operatorname{com} N=79$ e (c) e (d) $\operatorname{com} N=200$ para $P 2$ com $t_{c}=14$ dias e $t=24$ dias.

Para a Figura 4.16, apresenta-se amostragens com um melhor ajuste de P2 quando comparadas aos casos anteriores de $\theta$. Na Figura 4.16b, percebe-se novamente que o modelo de $L H S$ adere-se melhor no meta modelo. Uma última análise consiste nas Figura 4.16c e 4.16d, onde para ambos os cenários, o comportamento é o mesmo, ou seja, as PDFs dos meta modelos se ajustam entre si, porém esse ajuste não acontece comparandoos ao $M C^{R E F}$. Na Tabela 4.14 apresenta-se o ajuste de $G_{I}$ para $P 2$. 
Tabela 4.14 - Ajuste de $G_{I}$ para $P 2 \operatorname{com} t_{c}=14$ dias e $t=24$ dias

\begin{tabular}{c|c|c|c}
\hline \multirow{2}{*}{ Cenário } & Amostras & $\mathrm{N}$ & NRMSE \\
\hline$M C^{\text {REF }}$ & - & - & 1,00 \\
\hline \multirow{2}{*}{$C_{I}$} & $L H S$ & 79 & $-0,14$ \\
& $S S$ & 79 & $-0,56$ \\
\hline \multirow{2}{*}{$C_{I I}$} & $L H S$ & 79 & $-0,40$ \\
& $S S$ & 79 & $-0,14$ \\
\hline \multirow{2}{*}{$C_{I}$} & $L H S$ & 200 & $-0,10$ \\
& $S S$ & 200 & $-0,08$ \\
\multirow{2}{*}{$C_{I I}$} & $L H S$ & 200 & $-0,10$ \\
& $S S$ & 200 & $-0,10$ \\
\hline
\end{tabular}

Nota-se na Tabela 4.14 que NRMSE apresenta-se com melhor ajuste para $N=200$ sendo a diferença entre os cenários insignificante. Além disso, encontra-se na Figura 4.17 as PDFs para a interpolação por $R B F$.

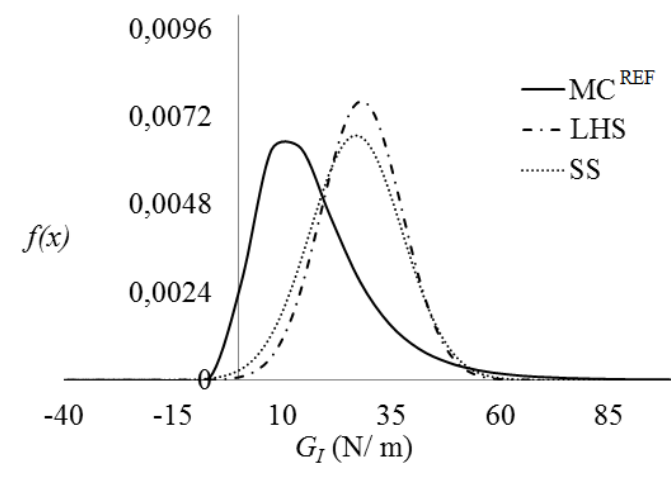

(a) $\mathrm{C}_{\mathrm{I}}, \mathrm{N}=79$

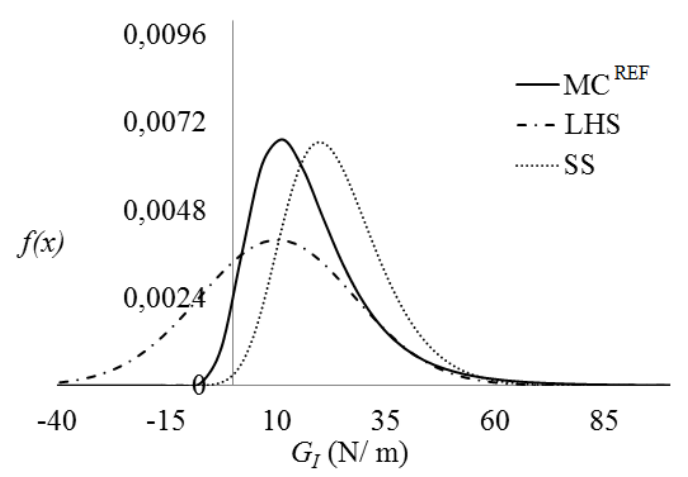

(c) $\mathrm{C}_{\mathrm{I}}, \mathrm{N}=200$

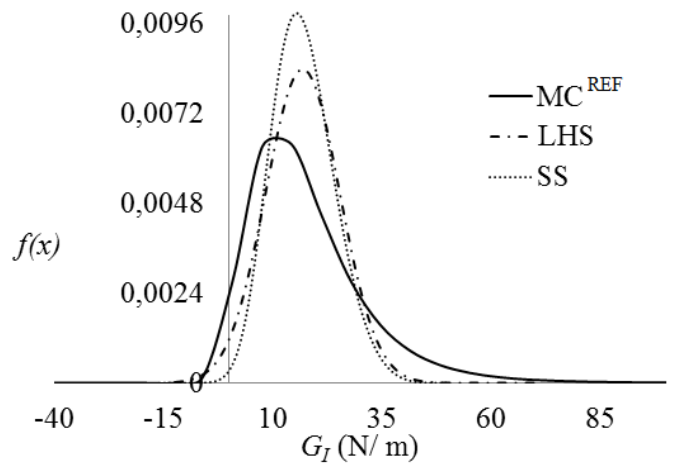

(b) $\mathrm{C}_{\mathrm{II}}, \mathrm{N}=79$

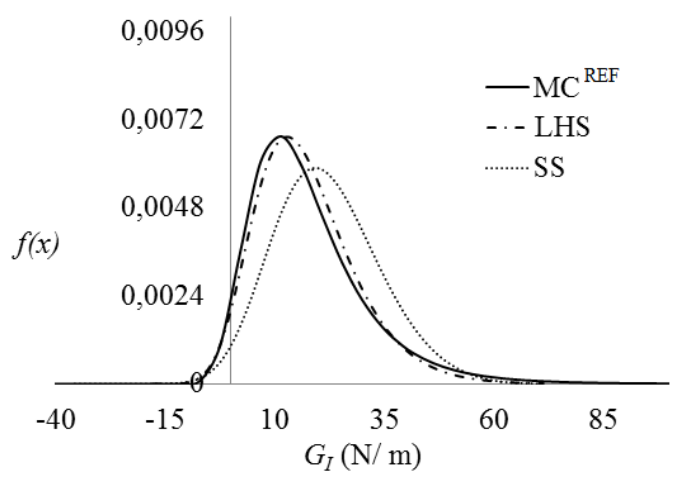

(d) $\mathrm{C}_{\mathrm{II}}, \mathrm{N}=200$

Figura 4.17 - $P D F s$ de $G_{I}$ sendo (a) e (b) com $N=79$ e (c) e (d) com $N=200$ para $R B F$ com $t_{c}=14$ dias e $t=24$ dias.

Analisando-se a Figura 4.17 observa-se o acoplamento das $P D F$ s para os cenários $\mathrm{C}_{\mathrm{I}}$ e $\mathrm{C}_{\mathrm{II}}$, em amostragens de $L H S$ e $S S$. Nota-se que o ajuste acontece para $C_{I I}$, tendo novamente LHS como o melhor resultado para $N=200$. Além disso, encontram-se na Tabela 4.15 os ajustes de NRMSE. 
Tabela 4.15 - Ajuste de $G_{I}$ para $R B F$ com $t_{c}=14$ dias e $t=24$ dias

\begin{tabular}{c|c|c|c}
\hline Cenário & Amostras & $\mathrm{N}$ & NRMSE \\
\hline$M C^{\text {REF }}$ & - & - & 1,00 \\
\hline \multirow{2}{*}{$C_{I}$} & $L H S$ & 79 & $-0,06$ \\
& $S S$ & 79 & $-0,08$ \\
\hline \multirow{2}{*}{$C_{I I}$} & $L H S$ & 79 & $-0,17$ \\
& $S S$ & 79 & $-0,11$ \\
\hline \multirow{2}{*}{$C_{I}$} & $L H S$ & 200 & $-0,28$ \\
& $S S$ & 200 & 0,00 \\
\multirow{2}{*}{$C_{I I}$} & $L H S$ & 200 & $-0,10$ \\
& $S S$ & 200 & $-0,14$ \\
\hline
\end{tabular}

Na Tabela 4.15 nota-se um melhor ajuste apenas para $N=79$ enquanto que para $N=200 \mathrm{o}$ ajuste é insignificante. Outra análise de $\theta$ consiste na adequação de um polinômio $P 1$ ao meta modelo dado na Figura 4.18.

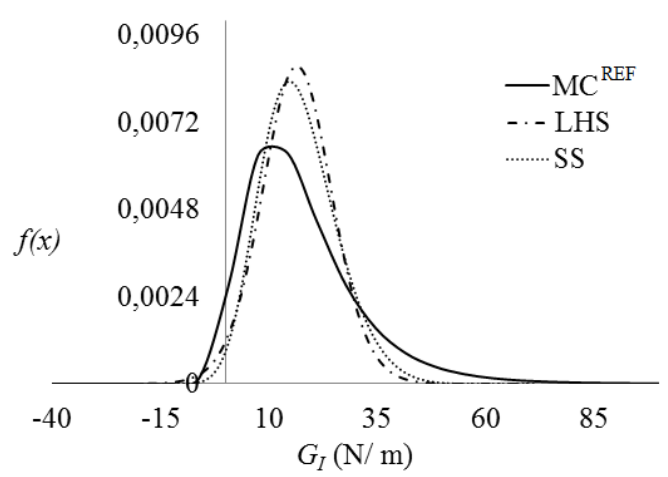

(a) $\mathrm{C}_{\mathrm{I}}, \mathrm{N}=79$

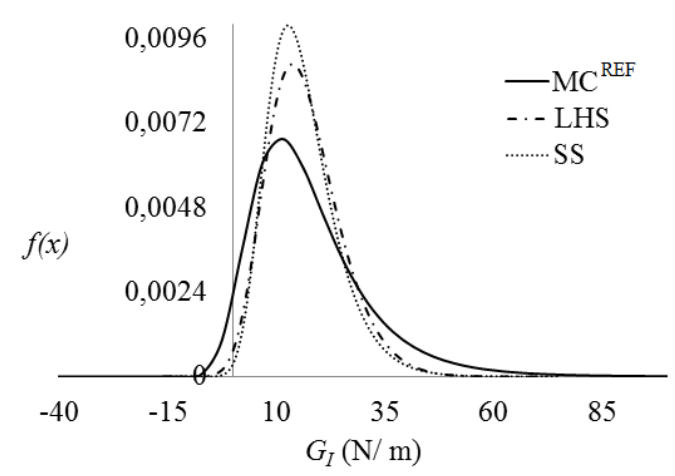

(c) $\mathrm{C}_{\mathrm{I}}, \mathrm{N}=200$

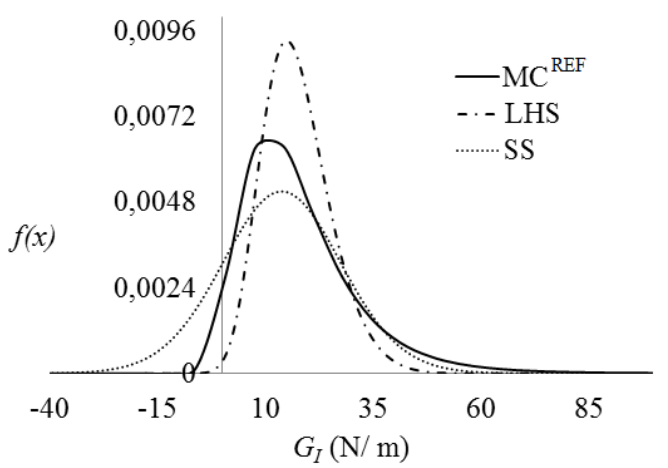

(b) $\mathrm{C}_{\mathrm{II}}, \mathrm{N}=79$

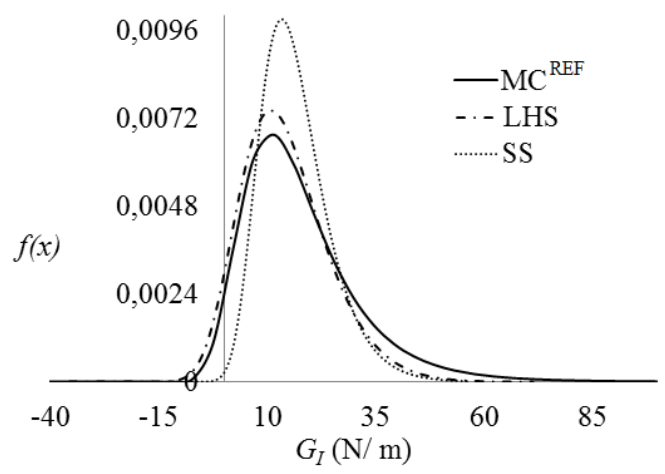

(d) $\mathrm{C}_{\mathrm{II}}, \mathrm{N}=200$

Figura $4.18-P D F s$ de $G_{I}$ sendo (a) e (b) $\operatorname{com} N=79$ e (c) e (d) $\operatorname{com} N=200$ para $R B F P I$ com $t_{c}=14$ dias e $t=24$ dias.

Na Figura 4.18 nota-se que as $P D F s$ dos meta modelos ajustam-se melhor a $M C^{R E F}$ quando comparados a $P 2$ e a $R B F$. Para $N>200$, observa-se que a Figura 4.18d apresenta para a amostragem de LHS o melhor comportamento. Por fim, conclui-se que para os meta modelos apresentados, a maioria segue a distribuição de valor extremo para $\delta=1$ e que o 
uso de um polinômio $P 1$ permite um melhor ajuste das funções densidade de probabilidade. Outra análise consiste na Tabela 4.16 para NRMSE em RBFP1.

Tabela 4.16 - Ajuste de $G_{I}$ para $R B F P 1 \operatorname{com} t_{c}=14$ dias e $t=24$ dias

\begin{tabular}{c|c|c|c}
\hline \multirow{2}{*}{ Cenário } & \multirow{2}{*}{ Amostras } & $\mathrm{N}$ & NRMSE \\
\hline$M C^{R E F}$ & - & - & 1,00 \\
\hline \multirow{2}{*}{$C_{I}$} & $L H S$ & 79 & $-0,17$ \\
& $S S$ & 79 & $-0,17$ \\
\hline \multirow{2}{*}{$C_{I I}$} & $L H S$ & 79 & $-0,08$ \\
& $S S$ & 79 & $-0,19$ \\
\hline \multirow{2}{*}{$C_{I}$} & $L H S$ & 200 & $-0,09$ \\
& $S S$ & 200 & $-0,10$ \\
\multirow{2}{*}{$C_{I I}$} & $L H S$ & 200 & $-0,14$ \\
& $S S$ & 200 & $-0,18$ \\
\hline
\end{tabular}

Nota-se na Tabela 4.16 que os dados apresentam-se em um intervalo praticamente semelhante e desta forma não há como concluir qual teve melhor reajuste.

\subsubsection{Análise da variação de $\delta$}

Para as Figuras 4.19 e 4.20 os valores de suporte $(\delta)$ variam de 0,5 a 2,0 sendo ambos apresentados para um número de amostras $N=79$ e com o cenário $C_{I}$ em amostragens de LHS e SS.

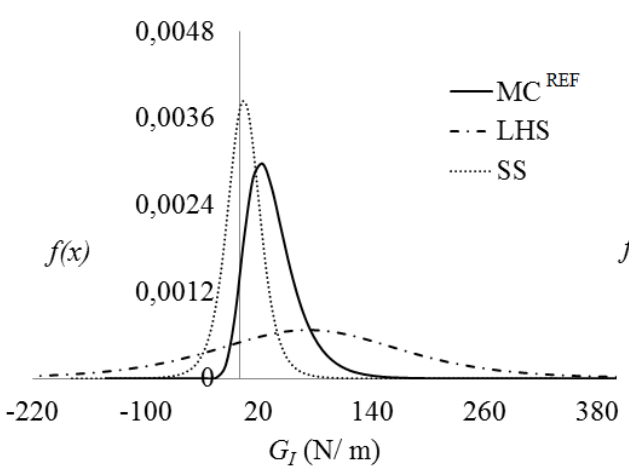

(a) $\delta=0,5$

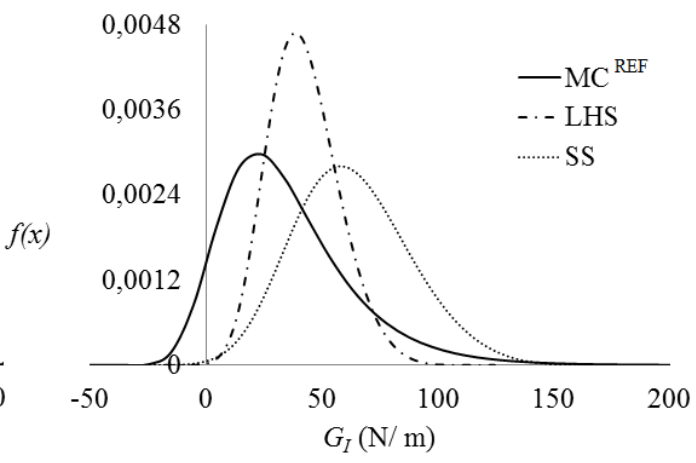

(b) $\delta=1,0$

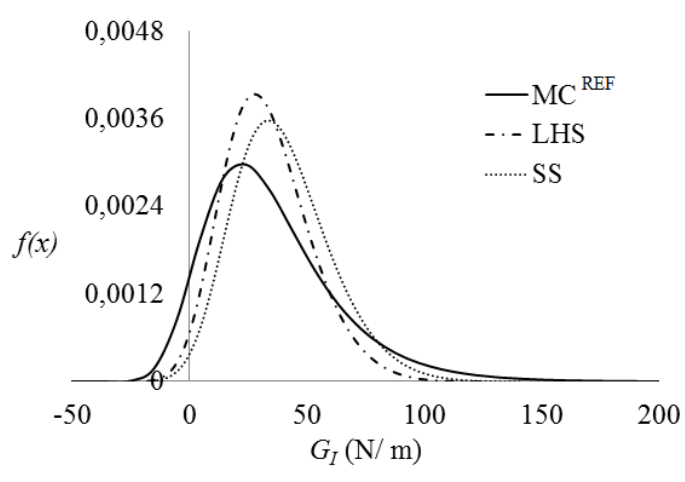

(c) $\delta=2,0$

Figura 4.19 - PDFs de $G_{I} \operatorname{com} N=79$ para análise de $\delta$ para $R B F \operatorname{com} t_{c}=1$ dia e $t=11$ dias; sendo (a) $\delta=0,5$; (b) $\delta=1,0$ e (c) $\delta=2,0$. 
Na Figura 4.19a, a amostragem por $L H S$ apresenta alta dispersão de forma a invalidar o meta modelo. Já para a Figura 4.19b, o nível de dispersão dos dados das PDFs é inferior comparado ao da Figura 4.19a, porém ainda insuficiente. Por fim, na Figura 4.19c, observa-se um ajuste mais adequado dos meta modelos. Desta forma, conclui-se que a medida que o suporte aumenta, o mesmo influencia consideravelmente nas PDFs dos meta modelos. Para RBFP1 apresenta-se a Figura $4.20 \mathrm{com}$ as $P D F s$ para os cenários $C_{I} \mathrm{e} C_{I I}$.

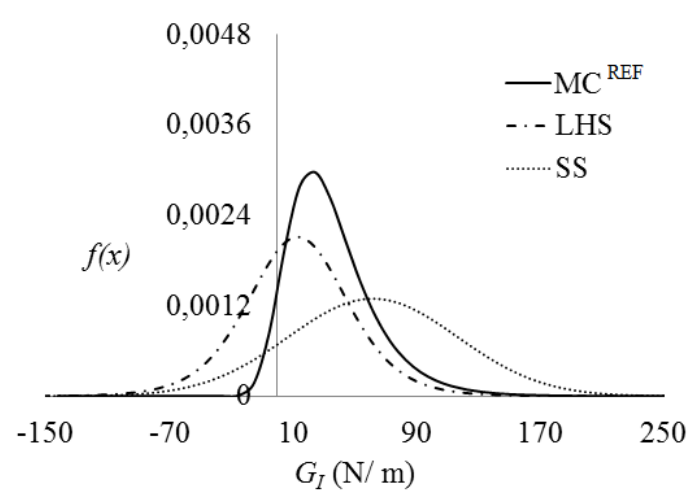

(a) $\delta=0,5$

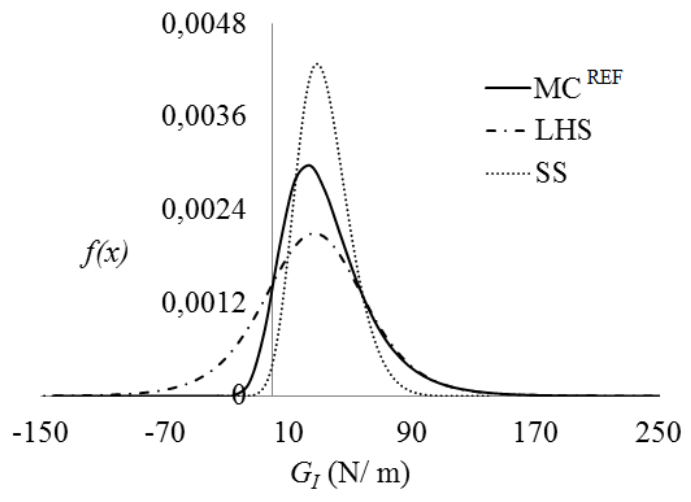

(b) $\delta=1,0$

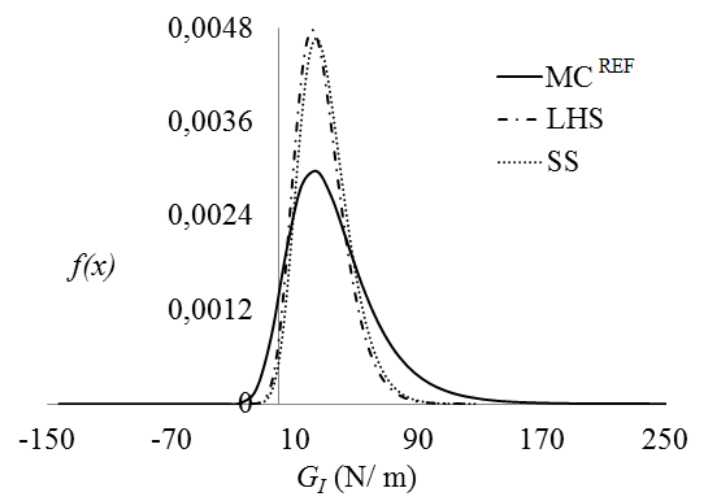

(c) $\delta=2,0$

Figura $4.20-P D F s$ de $G_{I} \operatorname{com} N=79$ para análise de $\delta$ para $R B F P 1$ com $t_{c}=1$ dia e $t=$ 11 dias; sendo (a) $\delta=0,5$; (b) $\delta=1,0$ e (c) $\delta=2,0$.

Uma última análise do suporte é realizada para a interpolação de $R B F P 1$, onde um polinômio $P 1$ é adicionado ao meta modelo. Na Figura 4.20a, percebe-se uma grande dispersão dos dados para $S S$ de $\delta=0,5$. Na Figura 4.20b, nota-se uma melhor aderência de $L H S$ ao modelo de referencia $M C^{R E F}$. Por fim, na Figura $4.20 \mathrm{c}$, percebe-se um melhor ajuste dos meta modelos.

\subsubsection{Análise Geral de $G_{I}$}

Uma última análise consiste na verificação dos melhores resultados para todos os casos de $\theta$, ou seja, para $P 2$ e $R B F P 1$. Para $N=79$, apresenta-se a Figura 4.21 com o cenário $C_{I I}$ em 
uma amostragem LHS para uma análise global dos melhores ajustes das PDFs apresentadas anteriormente. Além disso, nesta mesma seção, um novo conjunto de amostragens é utilizado posteriormente para o cenário $\mathrm{C}_{\mathrm{II}}$. Considerou-se para as novas amostragens a variação de $\delta=2$ e 3 em $R B F P 1$.

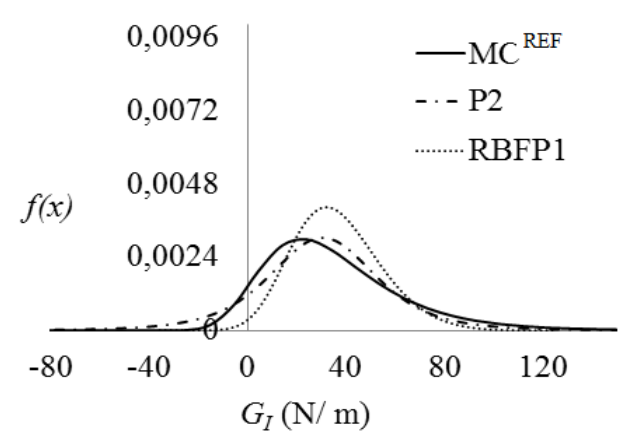

(a) $t_{c}=1$ dia e $t=11$ dias

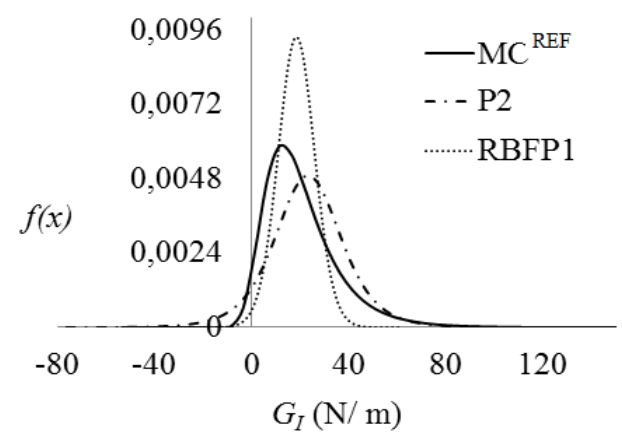

(b) $t_{c}=7$ dias e $t=17$ dias

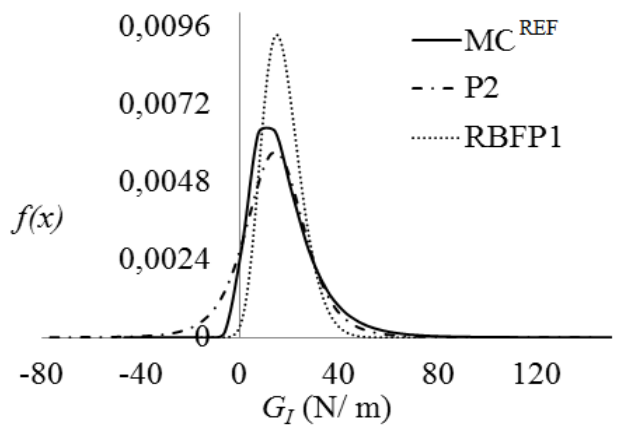

(c) $t_{c}=14$ dia e $t=24$ dias

Figura $4.21-P D F s$ de $G_{I}$ para $C_{I I} \operatorname{com} N=79$ sendo (a) $t_{c}=1$ dia e $t=11$ dias; (b) $t_{c}=7$ dias e $t=17$ dias e (c) $t_{c}=14$ dias e $t=24$ dias.

Na Figura 4.21 observa-se um melhor ajuste ao $M C^{R E F}$ para maiores valores de $t_{c}$. Nota-se um comportamento semelhante entre $P 2$ e RBFP1. Porém, analisando-se os NRMSE e os parâmetros de regressão conclui-se que RBFPl é mais eficiente. Na Figura 4.22 apresentam-se as funções densidades de probabilidade para $\mathrm{N}=200$ com cenário $C_{I I} \mathrm{e}$ amostragens LHS. 


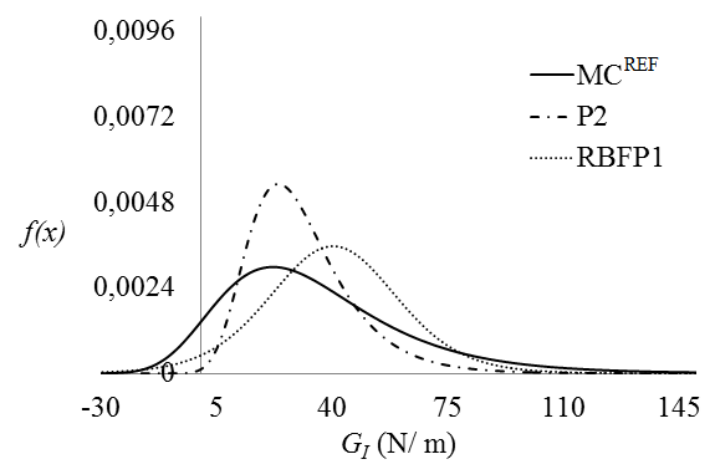

(a) $t_{c}=1$ dia e $t=11$ dias

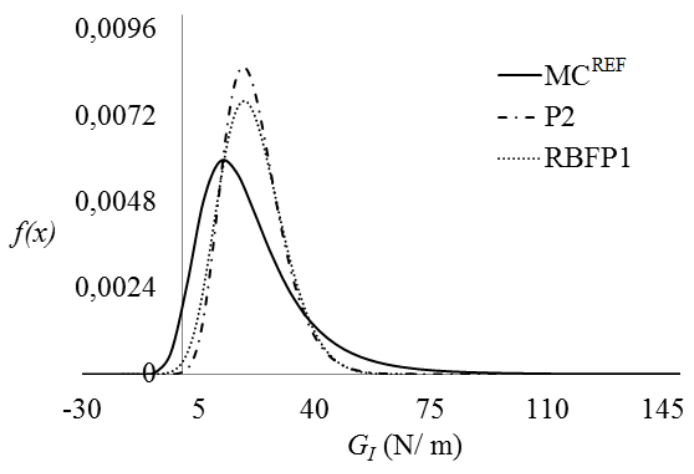

(b) $t_{c}=7$ dias e $t=17$ dias

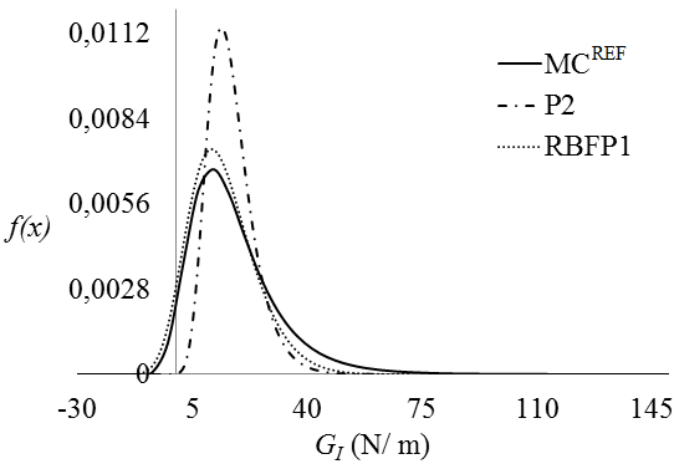

(c) $t_{c}=14$ dia e $t=24$ dias

Figura $4.22-P D F s$ de $G_{I}$ para $C_{I I} \operatorname{com} N=200$ sendo (a) $t_{c}=1$ dia e $t=11$ dias; (b) $t_{c}=7$ dias e $t=17$ dias e (c) $t_{c}=14$ dias e $t=24$ dias.

Na Figura 4.22 o melhor ajuste ocorre para $L H S$, sendo semelhante, portanto, a $N=79$. Além disso, nota-se que RBFPl apresenta melhores resultados de meta modelos para maiores valores de $t_{c}$. Uma última análise fora realizada na Figura 4.23 para o segundo caso de $\theta$ em diferentes valores de N para amostragens $L H S$ em RBFPl e P2.

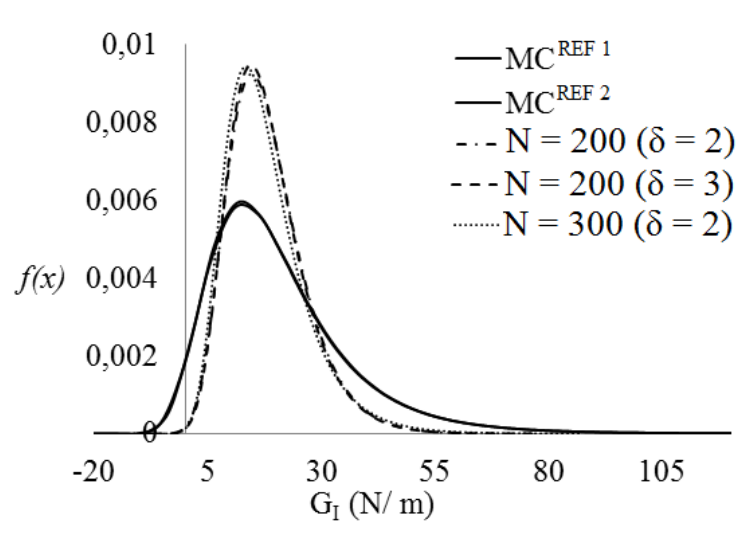

(a) $\mathrm{C}_{\mathrm{II}}, \mathrm{RBFP} 1$

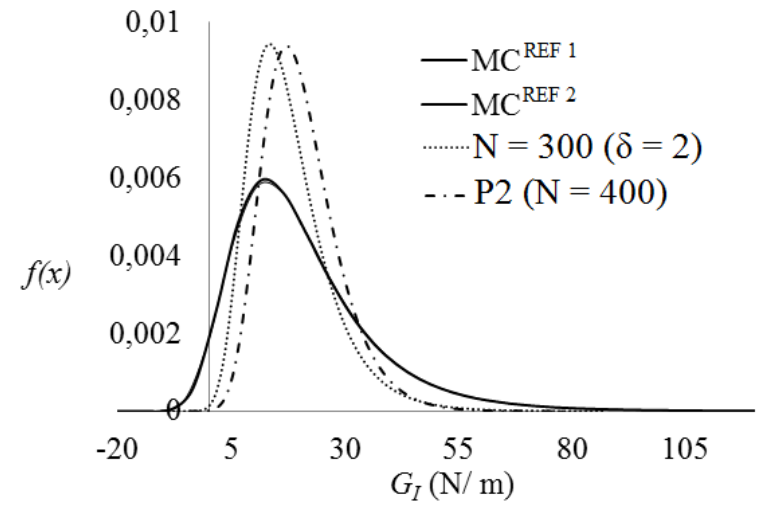

(b) $\mathrm{C}_{\mathrm{II}}, \mathrm{P} 2$ e RBFP1

Figura $4.23-P D F s$ de $G_{I}$ sendo com $t_{c}=7$ dias e $t=17$ dias. 
Na Figura 4.23 percebe-se que para $\delta>2$ a $P D F$ tende a apresentar um comportamento semelhante ao de $\delta=2$. Além disso, na figura, nota-se dois $\mathrm{MC}^{\mathrm{REF}}$ com objetivo de analisar o comportamento dos mesmos para NRMSE. Na Tabela 4.17 apresentam-se diferentes magnitudes de suporte para $L H S$ em função de um $M C^{R E F 1}$.

Tabela 4.17 - Ajuste de $G_{I}$ para $t_{c}=7$ dias e $t=17$ dias com $M C^{R E F 1}$

\begin{tabular}{c|c|c|c|c}
\hline \multirow{2}{*}{ Cenário } & Amostras & $\mathrm{N}$ & $\delta$ & NRMSE \\
\hline$M C^{R E F I}$ & - & - & - & 1,00 \\
\hline \multirow{3}{*}{$C_{I I}$} & $L H S(P 2)$ & 400 & - & 0,41 \\
& & 200 & 2,0 & 0,39 \\
& $L H S(R B F P 1)$ & 200 & 3,0 & $-0,12$ \\
& & 300 & 2,0 & 0,43 \\
\hline
\end{tabular}

Verifica-se na Tabela 4.17 um melhor ajuste das PDFs quando comparadas as adotadas anteriormente. Desta forma, percebe-se a necessidade de amostragens maiores que $N=$ 400. Já na Tabela 4.18 apresenta-se a segunda amostragem de $M C^{R E F}$.

Tabela 4.18 - Ajuste de $G_{I}$ para $t_{c}=7$ dias e $t=17$ dias com $M C^{R E F 2}$

\begin{tabular}{c|c|c|c|c}
\hline \multirow{2}{*}{ Cenário } & Amostras & $\mathrm{N}$ & $\delta$ & NRMSE \\
\hline$M C^{R E F 2}$ & - & - & - & $-0,41$ \\
\hline \multirow{3}{*}{$C_{I I}$} & $L H S(P 2)$ & 400 & - & $-0,10$ \\
& & 200 & 2,0 & $-0,12$ \\
& $L H S(R B F P 1)$ & 200 & 3,0 & 0,39 \\
& & 300 & 2,0 & $-0,13$ \\
\hline
\end{tabular}

Na Tabela 4.18 nota-se que mesmo ao gerar-se um novo $M C^{R E F}$, o fenômeno de análise continua com dificuldade para predição da incerteza. Porém, os resultados apresentados permitem uma aproximação da incerteza. 


\section{CONCLUSÕES E SUGESTÕES PARA TRABALHOS FUTUROS}

\subsection{CONCLUSÕES}

Esta dissertação quantificou a incerteza de fenômenos da mecânica da fratura e fadiga por meio de meta modelos. Para quantificar a incerteza, funções densidade de probabilidade foram utilizadas para o parâmetro de fadiga $N_{f}$ e para o parâmetro de fratura $G_{I}$.

Os resultados obtidos para as simulações do número de ciclos de vida a fadiga apresentaram-se mais eficientes com menor amostragem para mesma acurácia nos modelos propostos de $L H S$ e $S S$ tanto em $P 2, R B F$ e $R B F P 1$. No entanto, ao observarem-se as superfícies de respostas geradas a dois desvios padrões $(\sigma=2)$, verificou-se que a regressão satisfaz a todos os meta modelos propostos. Por fim, percebeu-se que o $L H S$ e o $S S$ estimados para a determinação das $P D F s$ apresentaram, para $\sigma<2$, valores próximos à média da distribuição.

Para quantificar a incerteza da taxa de liberação de energia, $G_{I}$, parâmetros adicionais foram regredidos da função fluência do concreto, sendo considerados três casos de $\theta$ adotados em amostragens aleatórias com cenários $C_{I}, C_{I I}$ e $C_{I I I}$. No primeiro caso de $\theta$ verificou-se que a regressão polinomial apresentava-se inadequada quando comparada ao processo de interpolação por RBFs. Além disso, percebeu-se que o uso de funções de base radial em conjunto a um polinômio de primeira ordem permitiu um melhor ajuste em termos de NRMSE das funções densidade de probabilidade para os cenários propostos. Contudo, notou-se ainda que o uso da amostragem por $L H S$ consiste, na maioria dos casos, na amostragem mais adequada para o número de simulações utilizadas.

No segundo e terceiro caso de $\theta$, os parâmetros regredidos de $P 2$ apresentaram melhores resultados para um número de amostras superior ao mínimo exigido. Já para a interpolação por funções de base radial, percebeu-se que os parâmetros de regressão apresentaram-se muito superiores em relação aos do processo polinomial. Contudo, ao verificarem-se as funções densidades de probabilidade, percebeu-se que os meta modelos de $R B F P 1$, foram os que mais se ajustaram ao modelo de referência, tendo melhores resultados para o segundo cenário que é com o número mínimo de amostras.

Conclui-se, por fim, que o uso de $R B F$ apresenta-se mais eficientes quando comparadas ao método de regressão polinomial. Contudo, a única desvantagem consiste apenas no tempo 
de processamento dos dados, pois o mesmo apresenta-se elevado quando comparado ao método polinomial.

\subsection{SUGESTÕES PARA TRABALHOS FUTUROS}

- Estudar possíveis correlações entre as variáveis, como por exemplo, entre os parâmetros geométricos e do material;

- Construir amostragens adaptativas para iterativamente melhorar a qualidade dos meta modelos;

- Realizar estudo de confiabilidade nos meta modelos propostos;

- Otimizar os processos de interpolação por funções de base radial, de forma a obterse menores tempos de processamento das simulações numéricas;

- Aplicar a metodologia a outros fenômenos estruturais, como por exemplo, em normas ou instruções de projeto de estruturas de concreto ou de aço;

- Quantificar a incerteza analisando-se o efeito escala na taxa de liberação de energia de estruturas de concreto armado;

- Calcular a incerteza para outros meta modelos da literatura, como por exemplo, utilizando o método de aproximação Moving Least Square (MLS). 


\section{REFERÊNCIAS BIBLIOGRÁFICAS}

Aalae, B., Abderrahmane, H., Gael, M. "Computational design of an automotive twist beam”. Journal of Computational Design and Engineering. p.p.: 215-225, Vol. 3, 2016.

Agrawal, R., Uddanwadiker, H., Padole, P. “Low Cycle Fatigue Life Prediction”. Journal of Emerging Engineering Research and Tecnology. p.p.: 5-15, Vol. 2, 2014.

Almeida, I. F., Evangelista Jr., F. "Quantificação da incerteza em meta modelos para predição de fadiga de baixo ciclo em membros estruturais." XXXVII CILAMCE, 14 p., Brasília, 2016.

Anderson, T. L. "Fracture Mechanics - Fundamentals and Applications". TAYLOR \& FRANCIS, Third Edition, 2005.

Atrushi, D. S. "Tensile and Compressive Creep of Early Age Concrete: Testing and Modelling”. Doctoral Thesis. Department of Civil Engineering. The Norwegian University of Science and Technology. Trondheim, Norway, 2003.

Badalló, P., Trias, D., Lindgaard, E. "Damage tolerance optimization of composite stringer run-out under tensile load”. Composite Structures. p.p.: 98-104, Vol. 133, 2015.

Bayramov, F., Taşdemir, C., Tadeşmir, M.A. "Optimization of steel fibre reinforced concretes by means of statistical response surface method". Cement \& Concrete Composites. p.p.: 665-675, Vol. 26, 2004.

Bierig, C., Chernov, A. "Approximation of probability density functions by the Multilevel Monte Carlo Maximum Entropy method”. Journal of Computational Physics. p.p.: 661681, Vol. 314, 2016.

Bigoni, D. "Uncertainty Quantification with Applications to Engineering Problems". Technical University of Denmar ${ }_{k}$. DTU Compute PHD - 2014, p.p.: 359.

Blatman, G. "Adaptive sparse polynomial chaos expansions for uncertainty propagation and sensitivity analysis”. Doctorate thesis. Université Blaise Pascal - Clermont II. Ecolé Doctorale Sciences pour I'Ingénieur de Clermont - Ferrand, 2009. 
Borges, J. F. "Confiabilidade e quantificação da incerteza da resistência à fratura considerando efeito de escala em vigas de concreto". Civil, Publicação E.DM-13A/16, Departamento de Engenharia Civil e Ambiental, Universidade de Brasília, Brasília, DF, 105 p., 2016.

Cao, L., Jiang, P., Chen, Z., Zhou, Q., Zhou, H. “Metamodel Assisted Robust Optimization under Interval Uncertainly Based on Reverse Model". International Federation of Automatic Control. p.p.: 1178-1183, 2015.

Choi, S. K., Grandhi, R. V., Canfield, R. A. "Reliability-based Structural Design". SPRINGER, LONDON, 2007.

Dey, P., Talukdar, S., Bordoloi, D.J. "Multiple-crack identification in a channel section steel beam using a combined response surface methodology and genetic algorithm". Structural Control and health monitoring. p.p.: 938-959, Vol. 23, 2016.

Dowling, N. E. "Mechanical Behavior of Materials - Engineering Methods for Deformation, Fracture, and Fatigue”. PERSON. p.p.: 416-790, 2013.

Dubourg, V., Sudret, B. “Meta-model-based importance sampling for reliability sensitivity analysis”. Structural Safety. p.p.: 27-36, Vol. 49, 2014.

Evangelista Jr., F., Muños P., I. D. “ Avaliação numérica do risco de falha estrutural por propagação de trincas em estruturas e materiais quase-frágeis incorporando incerteza e mecânica da fratura”. XXXV CILAMCE, 21p., Fortaleza, 2014.

Fang, J., Gao, Y., Sun, G., Xu, C., Li, Q. "Fatigue optimization with combined ensembles of surrogate modeling for a truck cab”. Journal of Mechanical Science and Technology. p.p.: 4641-4649, Vol. 28, 2014. DOI: 10.1007/s12206-014-1032-3.

Fasshauer, G. E. "Meshfree Approximation Methods with MATLAB". WORLD SCIENTIFIC. Illinois Institute of Technology, Vol. 6, 2007.

García, N. A., Evangelista Jr., F., Castro, F.C. "Quantificação da incerteza da vida de placas de aço sob à fadiga via polinômios de hermite." XXXVII CILAMCE, 12 p., Brasília, 2016. 
Gaspar, B., Teixeira, A. P., Soares, C. G. "Assessment of the efficiency of Kriging surrogate models for structural reliability analysis”. Probabilistic Engineering Mechanics. p.p.: 24-34, Vol. 37, 2014.

Gu, Y. T., Wang, W., Zhang, L. C., Feng, X. Q. “An enriched radial point interpolation method (e-RPIM) for analysis of crack tip fields". Engineering Fracture Mechanics. p.p.: 175-190, Vol. 78, 2011.

Hariri-Ardebili, M. A., Saouma, V. E. "Sensitivity and uncertainty quantification of the cohesive crack”. Engineering Fracture Mechanics. p.p.: 18-35, Vol. 155, 2016.

Kroetz, H. M. "Meta-Modelagem em confiabilidade estrutural”. Dissertação (Mestrado). Programa de Pós-Graduação em Engenharia de Estruturas. Escola de Engenharia de São Carlos da Universidade de São Paulo, 2015.

Leonel, E.D., Beck, A. T., Venturini, W.S. "On the performance of response surface and direct coupling approaches in solution of random crack propagation problems". Structural Safety. p.p.: 261-274, Vol. 33, 2011.

Li, X., Grace, J. R., Bi, X., Campbell, J. S. “A new pyrolysis model based on generalized extreme value (GEV) distributions and its application to lignocellulosic biomass". Fuel. p.p.: 211-221, Vol. 184, 2016.

Li, Z., Wang, W., Ismail, F. “A regulated boosting technique for material fatigue property prognostics”. Engineering Applications of Artificial Intelligence. p.p.: 153-159, Vol. 57, 2017.

Liu, G. R., Gu, Y. T. "Meshfree Shape Function Construction”. An Introduction to Meshfree Methods and Their Programming. p.p.: 54-144, 2005.

Lopez, R. H., Miguel, L. F. F., Cursi, J. E. S. "Uncertainty quantification for algebraic systems of equations". Computer and Structures. p.p.: 189-202, 2013.

Maneschy, J. E., Miranda, C. A. J. “Mecânica da Fratura na Indústria Nuclear”. Coleção Eletrobrás Eletronuclear, Eletrobrás, Rio de Janeiro, 2014.

Montgomery, D. C. “Design and Analysis of Experiments”. JOHN \& SONS, 2013. 
Myers, R. H., Montgomery, D. C., Cook, C. M. "Response Surface Methodology-Process and Product Optimization Using Designed Experiments”. NEW JERSEY, 2009.

Negrão, G. Q. “Implementação e avaliação da metodologia deformação-vida em componentes entalhados”. Dissertação de mestrado, Publicação ENM. Departamento de Engenharia Mecânica, Universidade de Brasília, DF, pp. 7-18, 2014.

Newman, J. C., Raju, I. S. "An empirical stress-intensity factor equation for the surface crack”. Engineering Fracture Mechanics. p.p.: 185-192, Vol. 15, 1981.

Pina, A. C., Albrecht, C.H., Lima, B. S. L. P., Jacob, B. P. “Wavelet network meta-models for the analysis of slender offshore structures". Engineering Structures. p.p.: 71-84, Vol. 68, 2014.

Regis, R. G. "Multi-objective constrained black-box optimization using radial basis function surrogates”. Journal of Computational Science. p.p.: 140-155, Vol. 16, 2016.

Reuter W. G., Underwood, J. H., Newman, J. C. "Surface-Crack Growth: Models, Experiments, and Structures". American Society for Testing Materials (ASTM), STP1060, 1990. DOI: 10.1520/STP1060-EB.

Rosenblatt, M. “Remarks on a Multivariate Transformation”. The annals of Mathematical Statistics. p.p. 470-472, Vol. 23, No. 3, 1952.

Sanford, R. J. "Principles of Fracture Mechanics". PERSON, NEW JERSEY, 2003. ISBN: 0-13-092992-1.

Santilli, A., Puente, I., Tanco, M. "A factorial design study to determine the significant parameters of fresh concrete lateral pressure and initial rate of pressure decay". Construction and Building Materials. p.p.: 1946-1955, Vol. 25, 2011.

Shields, M. D., Teferra K., Hapij, A., Daddazio, R. P. "Refined Stratified Sampling for efficient Monte Carlo based uncertainty quantification”. Reliability Engineering and System Safety. p.p. 310-325, Vol. 142, 2015.

Shields, M. D., Zhang J. “The generalization of Latin hypercube sampling”. Reliability Engineering and System Safety. p.p. 96-108, Vol. 148, 2016. 
Sobol, I. M. "On the distribution of points in a cube and the approximate evaluation of integrals”. USSR Computational Mathematics and Mathematical Physics. p.p. 86-112, Vol. 7, 1967.

Sudret, B. "Meta-models for structural reliability and uncertainty quantification". Asian Pacific Symposium on Structural Reliability and its Applications. Published by Research Publishing. p.p.: 1-7, 2012.

Tan, P. J. B., Hsu, M. H. "Crack effects on the dynamic behavior of orthotropic composite pre-twisted blades using radial basis function model”. 11th Conference on Industrial Electronics and Applications (ICIEA), 2016. DOI: 10.1109/ICIEA.2016.7603816.

Tsao, H. S. J., Patel, M. H. “A general intuitive pattern for optimally sequencing treatment combinations in $2^{k}$ factorial experiment and a simple estimation algorithm". Computers \& Industrial Engineering. p.p.: 423-436, Vol. 85, 2015.

Wahid, H., Ahmad, A. "A Computational Intelligence Approach to Solve the Inverse Problem of Electrical DC Resistivity Sounding”. Jurnal Teknologi (Sciences \& Engineering). p.p.: 115-120, 2014.

Wan H. P., Mao, Z., Todd, M. D., Ren, W. X. "Analytical uncertainty quantification for modal frequencies with structural parameter uncertainty using a Gaussian process metamodel”. Engineering Structures. p.p.: 577-589, Vol. 75, 2014.

Wang, Q., Fang, H., Shen, L. "Reliability analysis of tunnels using a metamodeling technique based on augmented radial basis functions". Tunnelling and Underground Space Technology. p.p.: 45-53, Vol. 56, 2016.

Wang, L., Chen, J. S., Hu, H. Y. "Subdomain radial basis function collocation method for fracture mechanics”. International Journal for Numerical Methods in Engineering. p.p.: 851-876, Vol. 83, 2010.

Wendland, H. "Piecewise polynomial, positive definite and compactly supported radial functions of minimal degree”. Advances in Computational Mathematics. p.p.: 389-396, Vol. 4, 1995. DOI: 10.1007/BF02123482. 
$\mathrm{Wu}, \mathrm{Z}$. "Compactly supported positive definite radial functions". Advances in Computational Mathematics. p.p.: 283-292, Vol. 4, 1995. DOI: 10.1007/BF03177517.

Xia, T., Yao, W., Xu, L., Zou, J. "Metamodel-based optimization of the bolted connection of wing spar considering fatigue resistence”. Journal of Aerospace Engineering. Institution of Mechanical Engineers. p.p.: 1-10, Vol. 230, 2015. DOI: 10.1177/0954410015598792.

Yuvaraj, P., Murthy, A.R., Iyer, N.R., Sekar, S.K., Samui, P. "Support vector regression based models to predict fracture characteristics of high strength and ultra high strength concrete beams”. Engineering Fracture Mechanics. p.p.: 29-43, Vol. 98, 2013.

Zhao, Y., Kou, H., Wu, W., Deng, Y., Tang, B., Li, J. "Prediction of High Cycle Fatigue Property of Ti-6Al-4V alloy Using Artificial Neural Network”. Materials Science Forum. p.p.: 360-367, Vol. 849, 2016. 
APÊNDICES 


\section{APÊNDICE A - PARÂMETROS DE REGRESSÃO PARA SEGUNDO CASO DE ANÁLISE DE G}

Nesta seção apresentam-se os parâmetros de regressão para $t_{c}=7$ dias $\operatorname{com} t=17$ dias. Os meta modelos são do tipo $P 2, R B F$ e $R B F P 1$, sendo o suporte $\delta=1$ e a função $R B F$ do tipo Wendland-C2.

Tabela A.1 - Parâmetros de $P 2$ para $t_{c}=7$ dias e $t=17$ dias

\begin{tabular}{ccccccc}
\hline \multirow{2}{*}{ Cenários } & $\begin{array}{c}\text { Regressão } \\
G_{I}\end{array}$ & SSE & RMSE & $\mathrm{r}^{2}$ & $\mathrm{~N}$ & \multirow{2}{*}{$\mathrm{NC}$} \\
\hline \multirow{2}{*}{$C_{I}$} & $L H S$ & $7,42 \mathrm{E}+1$ & 0,99 & 1,00 & 79 & 78 \\
& $S S$ & $6,64 \mathrm{E}+2$ & 0,99 & 1,00 & 79 & 78 \\
\hline \multirow{2}{*}{$C_{I I}$} & $L H S$ & $2,43 \mathrm{E}+2$ & 0,99 & 1,00 & 79 & 78 \\
& $S S$ & $2,57 \mathrm{E}+3$ & 0,99 & 1,00 & 79 & 78 \\
\hline \multirow{2}{*}{$C_{I}$} & $L H S$ & $2,24 \mathrm{E}+5$ & 0,87 & 1,00 & 200 & 78 \\
& $S S$ & $1,70 \mathrm{E}+5$ & 0,91 & 1,00 & 200 & 78 \\
\multirow{2}{*}{$C_{I I}$} & $L H S$ & $1,95 \mathrm{E}+5$ & 0,92 & 1,00 & 200 & 78 \\
& $S S$ & $1,64 \mathrm{E}+5$ & 0,91 & 1,00 & 200 & 78 \\
\hline
\end{tabular}

Tabela A.2 - Parâmetros de $R B F$ para $t_{c}=7$ dias e $t=17$ dias

\begin{tabular}{|c|c|c|c|c|c|c|}
\hline Cenários & $\begin{array}{c}\text { Regressão } \\
G_{I}\end{array}$ & SSE & RMSE & $\mathrm{r}^{2}$ & $\mathrm{~N}$ & $\mathrm{NC}$ \\
\hline \multirow[b]{2}{*}{$C_{I}$} & $L H S$ & 8,33 E-22 & $3,25 \mathrm{E}-12$ & 1,00 & 79 & 79 \\
\hline & $S S$ & 3,48 E-21 & 6,64 E-12 & 1,00 & 79 & 79 \\
\hline \multirow{2}{*}{$C_{I I}$} & $L H S$ & $1,05 \mathrm{E}-22$ & $1,15 \mathrm{E}-12$ & 1,00 & 79 & 79 \\
\hline & $S S$ & $3,50 \mathrm{E}-23$ & $6,66 \mathrm{E}-13$ & 1,00 & 79 & 79 \\
\hline \multirow{2}{*}{$C_{I}$} & $L H S$ & 6,73 E-23 & $5,80 \mathrm{E}-13$ & 1,00 & 200 & 200 \\
\hline & $S S$ & $6,60 \mathrm{E}-20$ & 1,82 E-11 & 1,00 & 200 & 200 \\
\hline \multirow{2}{*}{$C_{I I}$} & $L H S$ & $5,53 \mathrm{E}-23$ & $5,26 \mathrm{E}-13$ & 1,00 & 200 & 200 \\
\hline & $S S$ & 3,31 E-22 & $1,29 \mathrm{E}-12$ & 1,00 & 200 & 200 \\
\hline
\end{tabular}

Tabela A.3 - Parâmetros de RBFP1 para $t_{c}=7$ dias e $t=17$ dias

\begin{tabular}{ccccccc}
\hline \multirow{2}{*}{ Cenários } & $\begin{array}{c}\text { Regressão } \\
G_{I}\end{array}$ & SSE & RMSE & $\mathrm{r}^{2}$ & $\mathrm{~N}$ & \multirow{2}{*}{$\mathrm{NC}$} \\
\hline \multirow{2}{*}{$C_{I}$} & $L H S$ & $2,07 \mathrm{E}-22$ & $1,62 \mathrm{E}-12$ & 1,00 & 79 & 79 \\
& $S S$ & $5,83 \mathrm{E}-23$ & $8,59 \mathrm{E}-13$ & 1,00 & 79 & 79 \\
\hline \multirow{2}{*}{$C_{I I}$} & $L H S$ & $1,42 \mathrm{E}-22$ & $1,34 \mathrm{E}-12$ & 1,00 & 79 & 79 \\
& $S S$ & $1,45 \mathrm{E}-23$ & $4,28 \mathrm{E}-13$ & 1,00 & 79 & 79 \\
\hline \multirow{2}{*}{$C_{I}$} & $L H S$ & $2,17 \mathrm{E}-16$ & $1,04 \mathrm{E}-9$ & 1,00 & 200 & 200 \\
& $S S$ & $2,73 \mathrm{E}-21$ & $3,69 \mathrm{E}-12$ & 1,00 & 200 & 200 \\
\multirow{2}{*}{$C_{I I}$} & $L H S$ & $9,54 \mathrm{E}-21$ & $6,91 \mathrm{E}-12$ & 1,00 & 200 & 200 \\
& $S S$ & $8,51 \mathrm{E}-21$ & $6,52 \mathrm{E}-12$ & 1,00 & 200 & 200 \\
\hline
\end{tabular}




\section{APÊNDICE B - PARÂMETROS DE REGRESSÃO PARA TERCEIRO CASO DE $\mathbf{G}_{\mathbf{I}}$}

Nesta seção apresentam-se os parâmetros de regressão para $t_{c}=14$ dias com $t=24$ dias. Os meta modelos são do tipo $P 2, R B F$ e $R B F P 1$, sendo o suporte $\delta=1$ e a função $R B F$ do tipo Wendland-C2.

Tabela B.1 - Parâmetros de $P 2$ para $t_{c}=14$ dias e $t=24$ dias

\begin{tabular}{ccccccc}
\hline \multirow{2}{*}{ Cenários } & $\begin{array}{c}\text { Regressão } \\
G_{I}\end{array}$ & SSE & RMSE & $\mathrm{r}^{2}$ & $\mathrm{~N}$ & \multirow{2}{*}{ NC } \\
\hline \multirow{2}{*}{$C_{I}$} & $L H S$ & $1,80 \mathrm{E}+1$ & 0,48 & 1,00 & 79 & 78 \\
& $S S$ & $2,15 \mathrm{E}+1$ & 0,52 & 1,00 & 79 & 78 \\
\multirow{2}{*}{$C_{I I}$} & $L H S$ & $2,35 \mathrm{E}+2$ & 1,72 & 1,00 & 79 & 78 \\
& $S S$ & $0,49 \mathrm{E}-1$ & 0,25 & 1,00 & 79 & 78 \\
\hline \multirow{2}{*}{$C_{I}$} & $L H S$ & $1,23 \mathrm{E}+5$ & 24,81 & 1,00 & 200 & 78 \\
& $S S$ & $1,20 \mathrm{E}+5$ & 24,55 & 1,00 & 200 & 78 \\
\multirow{2}{*}{$C_{I I}$} & $L H S$ & $1,20 \mathrm{E}+5$ & 24,51 & 1,00 & 200 & 78 \\
& $S S$ & $1,03 \mathrm{E}+5$ & 22,65 & 1,00 & 200 & 78 \\
\hline
\end{tabular}

Tabela B. 2 - Parâmetros de $R B F$ para $t_{c}=14$ dias e $t=24$ dias

\begin{tabular}{ccccccc}
\hline \multirow{2}{*}{ Cenários } & $\begin{array}{c}\text { Regressão } \\
G_{I}\end{array}$ & SSE & RMSE & $\mathrm{r}^{2}$ & \multirow{2}{*}{$\mathrm{N}$} & \multirow{2}{*}{$\mathrm{NC}$} \\
\hline \multirow{2}{*}{$C_{I}$} & $L H S$ & $2,02 \mathrm{E}-24$ & $1,60 \mathrm{E}-13$ & 1,00 & 79 & 79 \\
& $S S$ & $1,43 \mathrm{E}-23$ & $4,26 \mathrm{E}-13$ & 1,00 & 79 & 79 \\
\hline \multirow{2}{*}{$C_{I I}$} & $L H S$ & $2,23 \mathrm{E}-24$ & $1,68 \mathrm{E}-13$ & 1,00 & 79 & 79 \\
& $S S$ & $9,55 \mathrm{E}-24$ & $3,48 \mathrm{E}-13$ & 1,00 & 79 & 79 \\
\hline \multirow{2}{*}{$C_{I}$} & $L H S$ & $9,06 \mathrm{E}-22$ & $2,13 \mathrm{E}-12$ & 1,00 & 200 & 200 \\
& $S S$ & $2,55 \mathrm{E}-23$ & $3,57 \mathrm{E}-13$ & 1,00 & 200 & 200 \\
\multirow{2}{*}{$C_{I I}$} & $L H S$ & $6,96 \mathrm{E}-23$ & $5,90 \mathrm{E}-13$ & 1,00 & 200 & 200 \\
& $S S$ & $5,32 \mathrm{E}-23$ & $5,16 \mathrm{E}-13$ & 1,00 & 200 & 200 \\
\hline
\end{tabular}

Tabela B.3 - Parâmetros de RBFP1 para $t_{c}=14$ dias e $t=24$ dias

\begin{tabular}{ccccccc}
\hline \multirow{2}{*}{ Cenários } & $\begin{array}{c}\text { Regressão } \\
G_{I}\end{array}$ & SSE & RMSE & $\mathrm{r}^{2}$ & $\mathrm{~N}$ & \multirow{2}{*}{$\mathrm{NC}$} \\
\hline \multirow{2}{*}{$C_{I}$} & $L H S$ & $5,36 \mathrm{E}-22$ & $2,60 \mathrm{E}-12$ & 1,00 & 79 & 79 \\
& $S S$ & $1,53 \mathrm{E}-23$ & $4,40 \mathrm{E}-13$ & 1,00 & 79 & 79 \\
\hline \multirow{2}{*}{$C_{I I}$} & $L H S$ & $1,74 \mathrm{E}-22$ & $1,48 \mathrm{E}-12$ & 1,00 & 79 & 79 \\
& $S S$ & $7,51 \mathrm{E}-23$ & $9,75 \mathrm{E}-13$ & 1,00 & 79 & 79 \\
\hline \multirow{2}{*}{$C_{I}$} & $L H S$ & $1,90 \mathrm{E}-21$ & $3,08 \mathrm{E}-12$ & 1,00 & 200 & 200 \\
& $S S$ & $1,16 \mathrm{E}-21$ & $2,41 \mathrm{E}-12$ & 1,00 & 200 & 200 \\
\multirow{2}{*}{$C_{I I}$} & $L H S$ & $6,08 \mathrm{E}-21$ & $5,51 \mathrm{E}-12$ & 1,00 & 200 & 200 \\
& $S S$ & $3,32 \mathrm{E}-21$ & $4,07 \mathrm{E}-12$ & 1,00 & 200 & 200 \\
\hline
\end{tabular}

